SB
994
$M>R_{3}$

ofinge 
(5) $=2 x=18$<smiles>C1CCC2C(C1)CC1CCC2C1</smiles>

7

(5) and 40

2at $x^{2}=$

3.

$y^{\prime}$

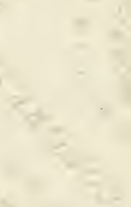

c. श्रa कon

5

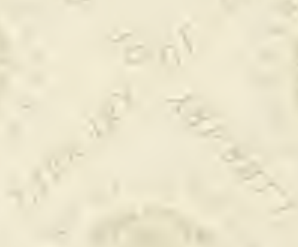

कर 9 
80

$4+1+x^{2}+2$

5

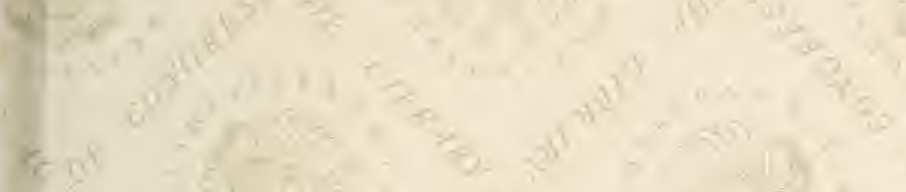

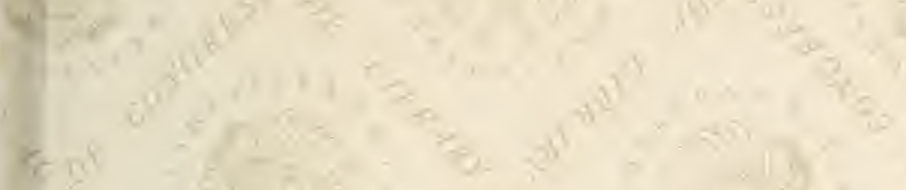

$8 \times 2$

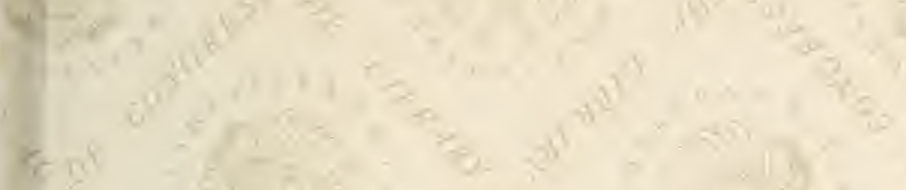

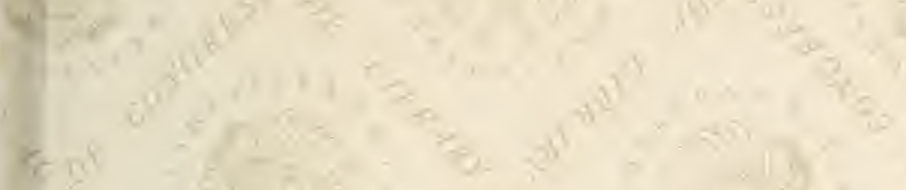

Mes

$6=$

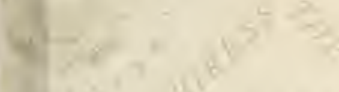

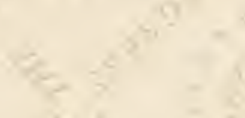

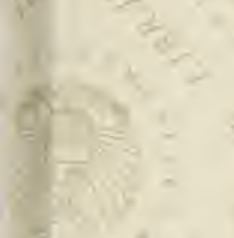

$(x+1,+2=4$

6.

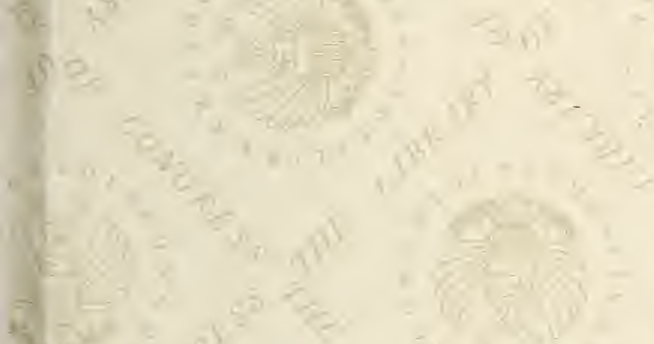

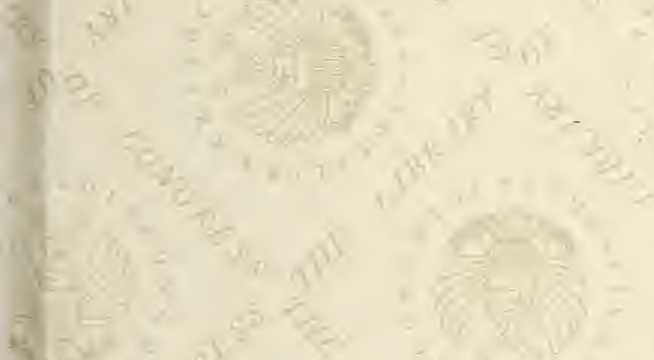

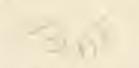

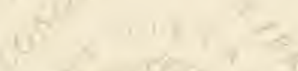

5.
15
6

$y^{2}=28$

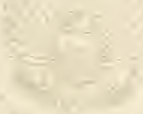

48

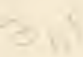

(i)<smiles>C1CCCCC1</smiles>

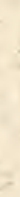

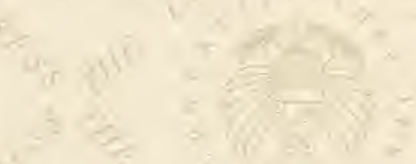

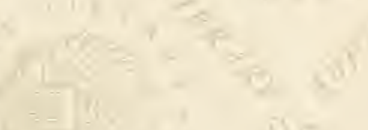
ive<smiles>C1=C2CCCC2CCCC1</smiles>

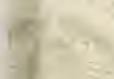

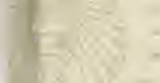

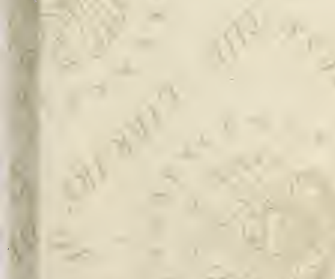

(1) -3

$x^{4}$

a)
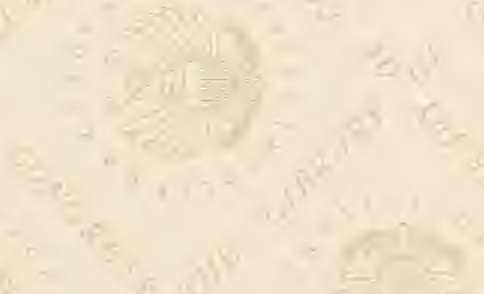







BNCYCLOPEDE-RORET

\section{LE CHASSEUR}

\section{TAUPIER.}




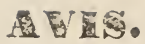

Le mérite des ourrages de l'Encyclopédie-Roret leur a ralu les homneurs de la traduction, de l'imitation et de la contrefaçon. Pour distinguer ce volume, il portera, à l'avenir, la véritable signature de l'Éditeur.

sition

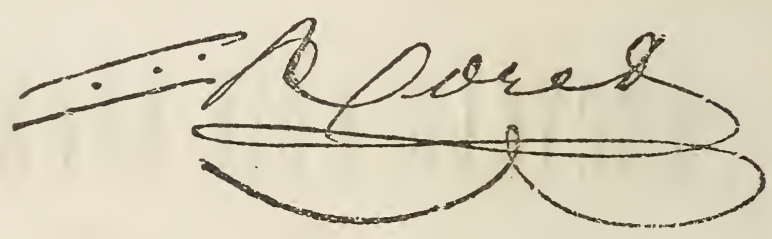

Cuez RoRet, hue Hiuvefeulle, 12.

ANINAUX NUISIBLES (Destructeur des) à l'agriculture, au jardinage, etc., par M. Verardi. 1 vol. orné de planches: prix. • • • • • . • . 3 tr.

2 Partie, contenant les HYLOPHTHIRES ET LEURS ENNEMIS, ou Description et Iconographie des Insectes les plus nuisibles aux forêts, avec une méthode pour apprendre à les détruire et à ménager ceux qui leur font la guerre, à l'usage des forestiers, des jardiniers, etc.; par Mi. Ratzeburg de Cobaeron el Boisduval, 1 vol. orné de 8 planches : prix. . . . . $2 \mathrm{fr} .50 \mathrm{c}$. 


\section{MANUELS-RORET.}

LE

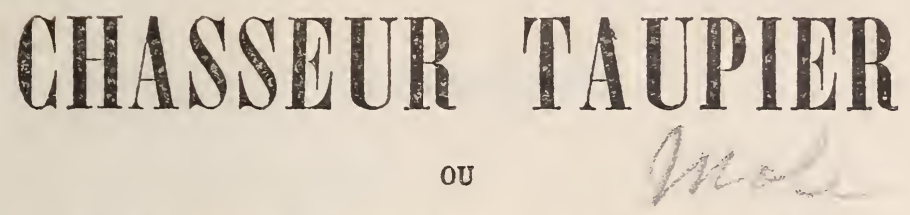

\section{L'ART DE PRENDRE LES TAUPES}

PAR DES HOTENS SURS ET FACILES;

PRÉCÉDÉ DE LEUR HISTOIRE NATURELLE.

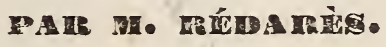

$$
\text { MOUVELLE ÉDITION, }
$$

AUGMENTLE D'UN TRAITE SUR TA DESTRUCTION DES ANIMAUX ET DES INSECTES NUISIBLES AU JARDINAGE.

Qurrage orné de figures.

\section{PARIS,}

1. LA LIBRAIRIE ENGIGLOPÉDIQUE DE RORET,

RUE HAUTEFEUTLL, 19.

1830 


$$
c s^{a^{2 x}}
$$




\section{LO CHISSUUR TAUPIER.}

\section{INTRODUCTION.}

Lorsqu'on veut étudier avec fruit les ouvrages dc la nature, on doit, avant tout, se persuader qu'elle n'a rien fait d'inutile, et que la vie du plus petit insecte est liée aux causes qui maintiennent l'harmonie universelle. Ce sentiment nous éloigne de l'indifférence que le commun des hommes a ordinairement pour la plupart des êtres qui, ail premier coup-d'œil, semblent avoir été créés sans nécessité; il nous rend plus sages et plus réservés dans nos jugemens, et nous inspire l'amour de la science et du travail.

Le vulgaire, qui ne juge que par ses yeux ou par ses préventions, décide de l'importance des obje:s qui le frappent, selon les rapports qu'ils ont avec lui, ou d'après l'influence qu'ils exercent sur son esprit. Le chasseur soutient que les hèvres et les lapins sont des êtres très utiles, qu'ils servent à nos plaisirs et à nos besoins; le laboureur proteste contre ce jugement, et dit que ces animaux n'ont été créés que pour dévaster les champs et détruire les récoltes; faites entendre à une dévote que les puces et les punaises n'ont reçu la vie ane pour nous tourmenter, s.fin 
de nous faire expier nos fautes, elle croira désormais que leur existence est nécessaire au salut des mortels.

Le naturaliste ne doit point s'arrêter sur de pareils jugemens; dépouillé de toute prévention et plein de confiance dans la puissance créatrice, il doit établir sa croyance sur des faits, et ne rien décider sans preuve; il doit, s'il n'est pas assez convaincu, attendre que l'expérience et le travail lui aicnt découvert la vérité; anticiper son jugement sur les ceuvres de la nature, est une témérité qui n’appartient qu'à l''ignorance et à l'orgueil.

De tous les animanx qui peuplent nos champs, il en est̂ „peu qui aient reçu tant de malédictions de la part des agriculteurs, que le petit quadrupède connu sous le nom de taupe ; il est vrai qu'il lcur cause des dégâts nombreux; au printemps, il bouleverse leurs semis, ronge et détruit les racines des jeunes arbres, attaque surtout celles des plantos potagères que l'on élève prématurément sur des couches, rend informe un terrain artistement et péniblement travaillé, en élevant à la surface, ces monticulesde terre émiettée, connus sous le nom de Taupinières; en été, et pendant les chaleurs, il déserte son domicile $€ t$ va s'établir sur les bords des eaux et dans les endroits frais, et c'est encore de nouveaux ravages à craindre, car la taupe fouilleuse perce les digues des étangs et des rivières, donne issue aux eaux et occasionne partout où elle fouille des inondations partielles, qui changent la nature du terrain et le rendenis souvent infertile.

Mais la taupe détruit les vêr's et les chenilles, cllo se 


\section{$-7-$}

nourrit des larves de ces insectes destructeurs qui, à l'approch de la belle saison, viennent dépouiller nos jardins et nos vergers de leurs fleurs et de leurverdure. Le trâail de la taupen'est pas toujours nuisible à l'agriculture, il porte à la surface du sol une terre vierge chargée de principes fertilisans, et les buttes à demi-sphériques que ces animaux élèvent en formant leurs galeries, répandues et dispersées, dans les années suivantes, sur le terrain, sont peut-être le meilleur de tous les engrais (1). Car, s'il faut en juger par l'expérience, c'est toujours aux endroits près des taupinières où la végétation $\mathrm{s} \in$ montre plus forte et plus active. La taupe, dit-on, est aussi radivore ; j'en conviens, mais rien ne nous dit qu'elle se plait à manger d'un grand nombre de racines, et, d'après l'étude que l'on a faite des habitudes de cet animal, tout nous porte à croire qu'il ne mange, en fait de racines, que les bulbes de la famille des colchiques, qui ne produisent rien, si ce n'est quatre ou cinq feuilles que presque tous nos animaux domestiques dédaignent; je ne déciderai pas si les taupes peuvent faire autant de bien à l'agriculteur qu'elles lui font de mal, mais il est prouvé que, dans certains cas, elles lui sont utiles, et je partage l'opinion de cet écrivain naturaliste (2), qui soutient que l'extermination de leur race serait plus unisible qu'avantageuse à nos campagnes; dans le fait, la taupe

(I) M. de Manchbausen, dans sou ourrase qui a pour titre : le Pire de fumille, dit que, bien loin d'être pernicicux aux prés, ces animaux $y$ sont très favorables, car non seulement ils mangent les vers et les insectes, mais encore leurs taupinières, quand on a soin de les répandre, servent d'engrais et renouvellent la terre affaiblie. Besc pense comme cet écrivain allemand.

(2) M. Demarest. 
se plait dans un sol léger et fertile, où la terre est meuble st facile à remuer; là aussi naissent ei se multiplient les haunetons et les chenilles; cet animal, en se logeant à côté de ces insectes ennemis de toute végétation, en fait sa pâture journalière et empêche leur trop grande multiplication. Il est à croire que si cela n'était pas ainsi, nous aurions plus souvent à nous plaindre du manque de nos récoltes. Toutefois, il en est de ces animaux comme de ceux qui se reproduisent trop, leur nombre importune et nuit, et la prudence et les intérêts même de l'agriculture commandent leur destruction.

Les taupes mahheureusement se multiplient trop vite, et l'endroit qu'elles adoptent est bientôt peuplé de leur nombreuse postérité ; comme elles sont infatigables et perzévérantes dans leur travail, elles ne tardent pas â couvrir le sol de taupinières, et à sillonner l'intérieur de la terre de mille détours plus ou moins spacieux, qui servent de retraites aux mulots, aux campagnols, aux loirs et eux belettes, et c'est encore un inconvénient de plus, car ces animaux, quoique insecivores, deviennent nuisibles et incommodes à l'agriculteur lorsqu'ils sont trop nombreux. Enfin les taupes, lorsqu'elles pullulent trop dans une contrée, peuvent miner et détruire les routes, rompre les digues, donner issue, comme nous l'avons dit, à des courans d'eau, arrêter le cours de la végétation en rongeant les racines nourricières, et en leur coupant toute communication avec l'intérieur de la terre : comme il est démontré que la plupart des dégâts de cette nature sont leur ourrage, on doit considérer l'drt du Taupier comme un art 
utile, et le rendre à jamais inséparable de la science agncole.

Les anciens s'occupèrent peu de la destruction des taupes, et nos aïeux se firent seulement un amusement de les éclaircir de lcurs champs lorsqu'elles leur étaient trop incommodes.

L'agriculture ayant fait, depuis une cinquantaine d'années, de rapides progrès, on a mis un peu plus de soin à méditer sur les causes qui peuvenť nuire à la prospérité de cette partie de la science humaine, qui est le premier de tous les arts, et on a reconnu que la famille des taupes était une de celles qui faisaient le plus de ravages dans nos champs.

Depuis lors une foule d'écrivains naturalistes se sont̂ occupés à étudier les mœurs et les habitudes de ces animaux, et chacun d'eux a consigné dans les journalix d'agriculture ou de sciences naturelles le fruit de ses expériences et de ses travaux; parmi eux on doit disiinguer Lafaille, Dralet, Cadet de Vaux, qui ont fait trois bons ourrages sur les taupes; maig ces auteurs se sont trop restreints dans leurs propres décourertes, et ont trop compté sur l'infaillibilité de leurs procédés; dans un ouvrage de ce g’enre, qui peut serrir à toute la classe agricole, on doit tout dire, car ce qui ne convient pas aux uns peut plaire aux autres, et tel qui n'a pas à sa disposition ni taupière de Lafaille, $\mathrm{n}^{\mathrm{i}}$ pinces de Lecourt, peut être fort aise d'apprendre qu'à certaines heures du jour, on peut aller surprendre la taupe dans son trarail et la détruire, soit arøc une houe ou une 
bêche, soit avee un chien qu'on dresse exprès, soit enfin avec un appàt empoisonné qu'on place à l'entrée de la demeure souterraine de l'animal.

Il y a des pays en France, surtout dans la partie méridionale, où l'agriculteur n'emploie pas un étranger pour détruire les taupes, il fait cet ouvrage lui-même lorsque le besoin l'exige, et certes lorsqu'il n'a pas de piége, cela ne l'empêche pas d'aller faire la chasse et de détruire l'animal qui l'importune; je trouve donc qu'il n'est pas à propos de s'en tenir exclusivement à une seule méthode, sur un art dont les moyens peuvent varier selon les lieux et les circonstances, sans pour cela que le but qu'on se propose soit manqué ; c'est ce qui m’a déterminé à faire un ouvrage dans lequel on trouvera, par ordre de chapitres, tont ce qui a été reconnu, jusqu'à ce jour, utile pour prendre les taupes; j’ai joint à chaque procédé le fruit de mes expériences et de mes observations, et j'ai cherché à ne rien omettre de tout ce qui peut être utile et nécessaire à mon sujet. 


\section{HISTOIRE NATURELLE}

\section{DE IA TAUPE.}

Le petit animal trapu et alerte, connu sous le nom de Taupe, habite toutes les contrées fertiles de la terre; ses mœurs, sa vie souterraine, les avantages qu'offre son pelage doux et soyeux, le firent remarquer par les naturalistes de toutes les époques....

Les anciens faisaient des chapeaux de peaux de taupes d'une extrême beanté; Agricola dit avoir vu des habits fourrés de la peau de ces animaux; Pline rapporte qu'on en faisait des couvertures dans une contrée du Péloponèse; Aristote a écrit que les taupes transportées à Lébadie refusaient de fouller la terre, tandis que dans le territoire d'Orchomène, elles bouleversaient les campagnes (1). L'infatigable naturaliste grec, qui.n'avait observé que le rattaupe de son pays, a dit encore que la taupe n’a pas

(r) M. Demarest, qui rapporte ce fait, remarque que Lébadie, en Béutic, est un pays moutueux, et que celui d"Orchanéne est plus plat. 
d'yeux; ceux qui ne connaissaient pas le rat-taupe, et qui ont écrit après Aristote, tels qu'Orus, Appollo et Appien , l'ont accusé d'ignorance. Voilà comment on juge sourent le génie et le savoir. Cependant, il n'y a pas longtemps que la science du naturaliste n'était établie que sur des doutes et des hypothèses, et il suffit de regarder un siècle en arrière de nous pour nous convaincre que l'erreur était, le plus souvent, le partage de celui qui voulait raisonner sur les ourrages de la nature. Peu de temps avant Limné, Fabricius et Buffon, on confondait dans un seul genre les petits quadrupèdes voisins et analogues au genie taupe; Smelin divisa ce genre en quatre espèces, saroir : la taupe d'Europe, la taupe d'Asie, la taupe à. longue queue et la taupe rouge; les naturalistes modernes ont séparé de ce genre trois espèces qui leur ont paru ofirir des caractères physiques différens; ils en ont fait deux genres connus sous les noms de chrysochlore et de condylure; la taupe d'Asie et la rouge appartiennent au genre chrysochlore, la taupe à longue queue réunie au sorex forme le genre condylure ; le sorte qu'on peut considérer maintenant la taupe commune d'Europe comme formant seule un genre, et ce n'est que de cette espèce d'animal que nous derons nous occuper.

La taupe commune, fg. $1^{\text {re }}$, ( Valpa Europea) forme un genre de mammifères de l'ordre des carnassiers et de la famille des insectivores. C'est un animal petit, trapu et comme arrondi, qui a environ cinq pouces de long; il a la tête large et terminée en pointe ; son nez avance de quatre ligres au-delà de la mâchoire supérieure, ses yeux sont 
très petits (1), sa bouche extrêmement fendue et fournic d'un nombre considérable de dents; il a, à chaque pied, cinq doigts armés d'ongles forts, surtout ceux de devant; son pelage est ordinairement d'un beau noir d'ébène, quelques contrées en nourrissent de blanches, de cendrées, de citrines; Pallas assure que les taupes de la Sibérie sont Flanches et plus grosses que les nôtres; enfin, les taupes d'Europe ofirent cinq variétés : $1^{\circ}$ la taupe tulgaire : son poil est d'un noir plus ou moins foncé; $2^{\circ}$ la taupe blanche: elle est commune en Hollande; $3^{\circ}$ la taupe du pays d'Aunis : son poil est roux fort clair, tirant sur la couleur de ventre de biclie, sans mélange ni tache; elle est plus grosse que la taupe commune; $4^{0}$ la taupe du territoire d'Alais, décrite par Lafaille : tout son pelage est d'une belle couleur de citron; on la trouve dans la partie du Bas-Languedoc qui touche aux Cévennes; 号 la taupe variée : ellc offre une variété de couleurs qui la rend curieuse et agréable à la vue; celles de l'Ost-Frise ont tout le corps parsemé de taches blanches et noires; Seba a fait mention le premier de cette espèce de taupe. Les zoologistes modernes ont beaucoup mieux étudié le genre taupe que ceux du second âge, et leurs travaux présentent un ensemble de faits assez remarquable. M. Cuvier, dans son règne animal, M. Demarest, dans les Dictionnaires d'Histoire et des Sciences naturelles, r'ont rien laissé à désirer sur la description physiologique et anatomique de ce quadrupède, et nous y

(1) Le docteur Brown, dans son Essai sur les errexrs populaires, dit que la tanpe a des yeux très imparfaits, et qu'elle y voit assez sculement pour distinguer la lumiàre; Sealiger ajoute a ecla que c'est toul simplement $e_{8}$ que verlait lo naiurs. 
renvoyons le lecteur qui voudrait faire une étude approfondie de la taupe. Avant ces auteurs, Buffon avait parlé du genre taupe en connaisseur habile; ce grand peintre de la nature, dont les tableaux sont si brillans et si animés, considère la taupe comme l'un des animaux les mieux partagés dans tout ce qui concerne les avantages physiques; après avoir montré avec quelle magnificence la nature l'a douée du sixième sens, il ajoute : " La taupe a de plus le toucher délicat, son poil est doux comme de la soie, elle a l'ouïe très fine, et de petites mains à cinq doigts, bien différentes des extrémités des pieds des autres animaux, et presque semblables aux mains de l'homme; beaucoup de force pour le volume de son corps, le cuir ferme, un embonpoint constant, un attachement vif et réciproque du mâle et de la femelle, de la crainte et du dégoût pour toute société, les douces habitudes du repos et de la solitude, l'art de se mettre en sûreté et de se faire en un instant un asile, un domicile, la facilité de l'étendre et d'y trouver. sans en sortir, une abondante nourriture; voilà sa nature, ses mœurs préférables sans doute à des qualités plus brillantes et plus incompatibles avec le bonheur que l'obscurité la plus profonde. " La taupe est un animal casanier , qui a des mours et des habitudes tout opposées à celles des quadrupèdes qui vivent comme elle dans l'intérieur de la terre; elle ne se plaît pas, comme le lapin, à la société de ses semblables, et n'est poỉnt, comme lui, attachée à l'asile qui l'a vue naître; elle craint l'air et le grand jour, voilà pourquoi sa retraite ne communique point directement avec l'atmosphère; elle craint également le froid et le chaud, elle s'enfonce plus profondément dans la terre en été et en 
hirer, selon quic la température est extrême; active et laborieuse, la taupe ne s'engourdit pas, comme on le dit, pendant les froids, mais, en hiver, elle a de longs intervalles de repos, elle est alors enserelie sous une voûte de terre sans issues, antour de laquelle elle circule comme un crimincl dans sa prison; pendant ce temps elle ne fouille et ne pousse la terre à l'extérieur que dans des temps doux et pour chercher sa subsistance; quand l'époque de ses amours est passée et que l'éducation de ses petits est faite, la taupe vit dans la retraite et l'isolement, ei elle fuit toute communication avec les individus de son genre; les variations de l'atmosphère, l'humidité ou la trop grande sécheresse, la font changer de domicile; en hiver elle habite les jardins et les couches de terre abritées; en automne elle recherche les lieux élevés et non sujets aux inondations; au printemps elle fréquente les prairies et les vallons; en été et dans les fortes chaleurs elle se plaît aux bords des rivières et des ruisseaux; mais quelle que soit la contrée qu'elle habite, elle choisit tonjours, pour fonder son domicile, une terre douce et de bonne qualité, où elle trouve abondamment des larves et des vers dont elle fait sa principale nourriture.

Les taupes, éminemment fouilleuses, se font en un instant une habitation, et c'est sans doute pourquoi elles mettent si peu d'importance à quitter celle qu'elles ont; leur force musculaire étant en grande partie dans les muscles releveurs de leur tête et dans leurs bras, elles creusent la terre avcc leurs pattes antérieures, la relèvent avec leur museau et la rejettent arec leurs mains à la surface du sol; 
ont-elles creusé la longueur de leur corps, elles se retournent et déblayent la terre au dehors; le mâle plus vigoureux tasse ct comprime cette terre, et de l'effort de son museau il la travaille et la fait sortir moulée et comme arrondie; cet ouvrage, qui sefait arec une étonnante activité, fait l'admiration de tous ceux qui se plaisent à étudier les mœurs de cet animal : pour exprimer le mourement que fait la taupe en poussant la terre avec ses pattes et son museau, les taupiers disent que la taupe souffle.

La demeure des taupes est une cavité, sourent circulaire, placée à deux ou trois pieds sous terre, ainsi que les longues allées qui y conduisent; toutes ces allées ont des communications entre elles; tantôt elles sont à la surface du sol, tantòt elles sont plus profondes; elles sont d'ordinaire parallèles à l'horizon du sol; les taupes, dans leurs demeures, ne restent point oisives, et soit le besoin de chercher leur nourriture, soit l'amour du irarail, elles multiplient leurs points de communications et leurs galeries; elles élèvent autour du foyer principal de nombreuses buttes, et il n'est pas étonnant de roir une taupe, dans un seul printemps, élever quinze à vingt taupinières; toutefois le plus ordinairement, une taupe, soit mâle, soit femelle, ne forme que de six à huit taupinières; les galeries et les taupinières faites par les mâles sont plus larges et plus grosses que celles des femelles, celles-ci sont plus nombreuses. Rien ne distingue mieux les vieilles taupes des jeunes que la différence de leurs ouvrages; celles-ci, sans s'assujettir aux travaux réfléchis d'un àge plus mûr, se lirrent entièrement au premior feu d'uвe jeunesse vaga- 
bonde et sans expérience, ardentes à la continuation d'un manège aussi bizarre que défectueux; clles ne font que de légères traînasses sans ordre et sans suite, et pour ne point s'arrêter, selon toute apparence, dans leur course rapide, ou perdre le temps à rejeter une terre incommode, elles effleurent la superficie, qui suffit à peine pour les couvrir.

Quatre à cinq grosses taupinières, rapprochées entre elles et fraîchement construites dans un endroit élevé, près d'une haie ou d'un buisson, indiquent le réduit souterrain où une femelle a déposé ses petits.

La taupe suit toujours les vers dans l'intérieur de la terre; en été et dans les temps de pluie elle vient se loger, comme eux, à fleur de terre; dans les temps froids ou secs elle les suit dans les cavités profondes où ils s'enfoncent, et on n'aperçoit plus les taupes vers la surface.

Les taupes ont l'ouie très fine, c'est pour cela qu'elles sont souvent interrompues dans leur ouvrage, car le moindre bruit, le moindre vent les fait rentrer dans leurs retraites; lorsqu'elles se sentent en danger, elles s'enfoncent en terre par un tuyau perpendiculaire d'un pied et demi de profondeur, qu'elles se sont creusé. Ces animaux ne poussent point la terre pendant les grandes pluies ni pendant les gelées, il n'y a que les grands vents qui ne les incommodent pas; ils ne travailient pas continuellement, mais leurs retours sont réguliers, ils ont lieu le matin an soleil levé, à midi, et le soir à la chute dn jour.

C'est dans une belle matince de printemps et après la żosée que l'on voit la taupe dans son plus orand travail et 
que l'on peut juger de son activité; soit mâle ou femelle, soit jeune ou vieux, l'ouvrier qui est à l'ouvrage fouille avec une célérité extrême et rejette la terre avec tant de précipitation, que l'on ne pourrait cromre, si on n'en avait la certitude, que c'est la main d'une taupe qui opère un tel bouleversement. Aussi habile que volage, dit Lafaille, cet animal transporte à chaque instant son domicile d'un lieu à un autre; pour cet effet il franchit tout obstacle, murs, fossés, canaux, et pour éviter de périr au milieu des flots ou de consumer ses forces contre des retranchemens qui lui coupent souvent le passage, il sait, par une industrie merveilleuse, conduire ses galeries à une profondeur très grande sous les rivières et sous des fondemens épais.

Rencontre-t-il un obstacle insurmontable, en ingénieur habile, on le voit sonder les voies et le terrain, tourner autour des rochers ou des montagnes, et employer toutes les ressources de son génie pour se frayer un chemin; si on endommage ou les boyaux ou les amas de terre qu'il a formés, il vient tout aussitôt les réparer.

Les taupes craignent l'eau, elles ont cela de commun avec presque tous les quadrupèdes qui, comme elles, hahitent l'intérieur de la terre; lorsque quelques filières d'eau inondent ou rendent humide le lieu qu'elles se sont choisi, elles vont se creuser ailleurs un autre domicile; si les inondations et les débordemens des rivières les surprennent, on les voit fuir et faire de vains efforts pour se sauver, la plupart périssent au bout d'un quart d'heure, cédant à la fatiguc et à leur propre poids; cette mort violente produit dans les taupes un sentiment de désespoir qui 
va jusqu’à la fureur; irritées par l'eau qu'elles ne peuvent vaincre, on les voit tourner, revenir, s'inquiéter, et ne finir cet exercice qu'en soufflant contre le fluide exterminateur; leurs petits, qui restent dans les trous, périssent aussi ; sans cela, dit Buffon, les grands talents qu'elles ont pour la multiplication nous deviendraient trop incommodes.

En automne, la taupe se cantonne, elle se fait un véritable établissement, et elle ne tarde pas à s'y faire un gîte, d'où elle ne sort qu'au printempspour courir après de nouvelles amours et de nouveaux plaisirs.

La demeure habituelle des taupes est toujours ferinée lorsqu'elles ne sont pas à l'ourrage; elles sortent rarement, leurs promenades sont fort courtes et leurs sorties n'ont lieı que dans les beaux jours et par un temps doux; dans les belles nuits d'été, elles sortent parfois avec leurs petits qu'elles font courir et avec lesquels elles se plaisent, mais au moindre bruit elles fuient avec précipitation dans leur's réduits.

La taupe court, sous terre, avec assez de rapidité; sur le sol, elle court vite lorsque la terre est douce et légère; mais sur une surface sèche et aride, elle se meut péniblement, et il lui est impossible d'échapper à l'ennemi qui la poursuit; aussi on ne la voit jamais sur un terrain sec et dur, pas plus que dans les fonds humides et marécageux, ni dans les forêts; en général, tout terrain qui offre de la résistance à être exploité par ses mains ne lui convient pas; c'est dans les jachères et dans les prés qu'on la trauve le 
plus en nombre; les terres fraîchement labourées ou celles qui le sont trop souvent, ne voient presque jamais de taupinières, et cela s'explique assez facilcment: la crainte et la timidité naturelles de la taupe l'éloignent des lieux qui sont sans cesse foulés par les hommes ou par les animaux.

Malgré leur vie solitaire et ragabonde, les taupes ne se cherchent jamais querelle, mais à l'époque de leurs amours, une jalouse rivalité s'établit entre les mâles; ils se disputent avec acharnement les femelles, et ce sont toujours les plus forts qui sont les plus heurtux. Ces animaux, qui sarent se respecter sans se craindre, se dévorent pourtant lorsqu'ils se trouvent réunis dans un petit espacc d'où ils ne peuvent sortir. Lecourt fait mention de douze taupes ainsi renfermées, qui se dérorèrent toutes les unes après les autres; ll n'en restait qu'une le jour suivant.

Les taupes ont peu d'ennemis parmi les quadrupèdes ; mortes, nul animal n'en fait sa nourriture. Quelques auteurs ont avancé que lorsque les taupes perdaient la vie sur le sol, elles trouvaient des amis généreux, parmiles aninaux, qui leur donnaient la sépulture; Gleditsch a soutenu cette singularité dans un mémoire qui fait partie de l'histoire de l'Académie royale des sciences et belles-lettres de Berlin, année 1782. Mais les naturalistes modernes n'ont rien dit encore qui puisse y faire attacher quelque croyance.

Les taupes entrent en amour sur la fin de l'hiver; alors les mâles et les femelles se recherchent avec ardeur, et plus il s'offre d'obstacles à leur réunion, plus elles montrent le désir de se réunir. Cet état ardent que fait naître le besoin de se propager, et que prolongent les obstacles 
que présente une vie solitaire et isolée, ne dure pourtant qu'un instant pour les individus qui sont satisfaits, et la cohabitation des deux sexes se borne à l'intervalle qui s'ócoule dans l'acte du plaisir ; mais les accouplemens se font depuis le commencement de mars jusqu'à la fin de juin, pour la première portée; et pour la seconde, depuis juin jusqu'en août. Les zoologistes n'ont pas encore déterminé le temps de la gestation des femelles, tous s'accordent avec Buffon à penser qu'elle est de courte durée; ce qui fait croire äinsi, c'est que l'on trouve des petits au commencement de mars, et qu'on en trouve après jusqu'en août; les femelles font deux portées par an, de quatre à cinq petits chaque; elles sont en état d'être fécondées à leur premier printemps; on a remarqué qu'elles faisaient plus de mâles que defemelles. D'après ce que dit Cadet de Vaux, il paraît que les taupes mâles font des traces d'une nouvelle construction à l'époque de leurs amours; ces traces sont sans issues, l'intention de l'ouvrier, dit l'auteur que je cite, étant d'y enfermer la femelle et d'en interdire l'accès à ses rivaux; elles sont aussi d'une longueur extraordinaire; Lecourt en a vu une de quatre-vingt-quatorze toises. L'accouplement se fait de nuit et dans les traces où le couple demeure; quelque temps avant que la taupe mette bas, elle compose un domicile séparé et commode pour loger sa progéniture. Buffon le décrit ainsi: «Elles commencent par élever et former une voûte assez haute, ellespressent et battent la terre, la mêlent avec des racines et des herbes, et la rendent si dure et si solide par dessous, que l'eau ne peut pas y pénétrer; elles élèvent un tertre au-dedans, au sommet diantel elles apportent de l'herbe et des fenilles pour 
faire un lit à leurs petits; dans cette situation ils se trouvent au-dessus du niveau du terrain, et par conséquent à l'abri des inondations ordinaires, et en même temps à couvert de la pluie par la voûte qui recouvre le tertre sous lequel ils reposent : ce tertre est percé tout autour de plusieurs trous en pente, qui descendent plus bas et s'étendent de tous côtés, comme autant de routes souterraines par où la mère-taupe peut sortir et aller chercher la subsistance néccssaire à ses petits. Ces sentiers souterrains sont fermes et battus, s'étendent à douze ou quinze pas, et partent tous du domicile comme des rayons d'un centre; on y trouve, aussi bien que sous la voûte, des débris d'ognons de colchique, qui sont apparemment la première nourriture qu'elle donne à ses petits. )

C'est dans cet asile, si artistement rangé, que les femelles mettent las leurs petits, qui naissent nus et tout rouges; les petits, au bout de quelques jours, se couvrent de poils, et ils ne tardent pas à sortir de leur nid par une des galeries qui y aboutissent, et s'échappent par des traces vers la surface de la terre; souvent leur mère les entraîne avec elle et cherche à les faire courir, à les accoutumer au travail et à trouver leur subsistance.

Les mères-taupes ont beaucoup de tendresse et d'attachement pour leurs petits, elles ne les quittent, pendant les premiers jours de leur naissance, que pour leur aller chercher la nourriture, qui ne se compose que de feuilles teridres, des ognons de colchique. Les jeunes taupes travaillent la première année de leur naissance, et le printemps suivant elles sont on état de reproduire. Si les inon- 


\section{$-25-$}

dations, les oiseaux de proic et les carnassiers ne réduisaient pas le nombre des taupes, il y a tout à croire que leur postérité serait bíen plus nombreuse, et qu'elles nous deviendraient bien plus incommodes, car ces animaux, qui ne sont point sortis de leurs attributions naturelles, vivent dans un état permanent de santé, d'aisance et de bonheur.

Les taupes, comme le dit un auteur, déjà heureuses par la nature de leur demeure, le sont encore par la consistance de leur vêtement, la délicatesse de leurs sens, la perfection de leur instinct, la tranquillité dont elles jouissent, l'abondance de la nourriture, et surtont l'amour du travail, source assurée de contentement et d'aisance; sans doute ces animaux n'auraient rien à envier au reste des êtres, si une habitude naturelle ne les portait à détruire nos récoltes et à dévaster nos champs; ils se font par-là les ennemis des laboureurs, et le plus grand nombre périt par leurs mains, arant d'avoir parcouru le court et fortuné période de leur vie naturelle. 
DES GONMAISSANCES RÉCESSAIRES POUR FAIRE AVANTAGEUSBMENT

LA CHASSE AUX TAUPES。

C'est toujours la faute d'un propriétaire ou d'un jardinier, dit Rozier, si ses champs et ses prairies sont infectés de taupes; l'animal a beau être fin, avoir l'ouïc délicate, il est facile de le détruire, même sans taupières; je partage l'opinion de cet écrivain agronome, et je suis persuadé que, sans beaucoup de peine et avec un peu de connaissance sur les habitudes des taupes, un cultivateur peut facilement en débarrasser son héritage. D'abord cet animal a une saison, un temps, une heure fixés pour son travail; ses amours ont lieu aux mêmes époques; les soins qu'il porte à ses petits ont un terme court; on peut aussi apprécier l'étendue et la forme de son domicile, la distribution ou l'encadrement de ses galeries, la forme, la grosseur de ses taupinières : ces connaissances préliminaires mettent le taupier à même d'attaquer à propos el de dresser convenablement ses batteries.

Les taupes ont une liabitude constante ou plutôt une manière d'opérer, dans leur travail, qui décèle tonjours leur présence et facilice les moyens d'extermination. Si l'on ouvre, dit M. Dralet (1), avec un instrument tianchant, un boyau que la taupe a formé depuis per, elle vicnt quel- 
ques instans après le réparer, afin de se mettre à couvert du danger et du grand air; pour y parvenir, elle forme, à l'endroit ouvert, une route de terre mobilisée qui présente, à l'extérieur, une taupinière oblongue, au moyen de laquelle elle réunit et rapièce, pour ainsi dire, le boyau coupé. Si on endommage une taupinière fraîche, la taupe vient aussi réparer le dommage. Ces deux points de fait, ajoute cet écrivain, sont la base principale de l'art du taupier (2).

Ainsi, pour faire avantageusement la chasse aux taupes, il faut connaître les endroits où elles se plaisent, la saison où elles fouillent le plus la terre, le temps et l'heure de leur travail, la marche et la direction de leur ourrage, et juger, à l'inspection des taupinières, si l'animal est dans son réduit ou s'il l'a abandonné, et dans quel point il se trouve pour l'attaquer.

La taupe est craintive et alerte, elle fuit et s'enfonce dans son trou au moindre bruit qu'elle entend ; le meilleur moyen pour la surprendre est de la guetter en silence; lors donc que l'on se trouve dans une pièce de terre que les tanpes habitent, on examine d'un peu loin les buttes à demisphériques qu'elles ont formées, on fixe ses regards sur celles qui sont fraîchement élevées; souvent on aperçoit, non pas l'animal, mais ses mouvemens et la terre qu'elle rejette à la surface du sol, alors on s'approche furtivement de la taupinière, et l'on reste immobile et silencieux jus-

(2) Il faut entendre l'art de celui qui snit la méthode de M. Dralet, la base. del'Art du taupier, et la connaissance approfondie des habitades, des mours et des travaux des taupes, ot les moyens d'en tirer parti pour lesstrpreadr ot les détruire. 
qu'à ce que l'animal recommence son travail, afin de l'attaquer à coup sûr.

Dans les endroits fertiles et cultivés, la taupe fouille partout, mais, comme nous l'avons dit, les terres douces et profondes, dépouillées de pierres, de cailloux et de grosses racines, sont celles où elle se plaît le mieux; aussi la voit-on plus communément dans les jaruins, dans les vallons êt les prairies, au bord 'des haies, des Luissons et des grandes routes, sur les terres en friches ou en jachères; c'est par tous ces endroits que le taupier doit aller les chercher et établir ses piéges.

La taupe fouille la terre toute l'année pour chercher sa nourriture, mais rarement on la voit en hiver élever des taupinières; quelquefois, seulement, quand la température est douce et le temps calme, elle se permet d'effleurer la surface du sol et de pousser la terre à l'extérieur : ces exemples ne sont pas communs. Si l'automne est pluvieux et froid, la taupe reste encore dans l'intérieur de la terre, elle en agit de même dans les grandes chaleurs; la saison où elle travaille le plus, c'est le printemps, depuis le mois de mars jusqu'à la fin de juin, et proportionnément depuis le commencement de juillet jusqu'à la fin d'octobre; une température plus ou moins inconstante ralentit ou augmente la vigueur des taupes pour l'ouvrage. Les taupes, dans le printemps, sont poussées par un fort aiguillon à creuser et à bouleverser les terres qu'elles habitent; d'abord le besoin dominateur de se reproduire, qui les oblige de se rechercher mutucllement; ensuite la nécessité d'établir un domicile pour leurs petits et de chercher leur nourri- 


\section{$-27-$}

ture; c'est done spécialement à cette époque qu'il faut faire la guerre à ces animaux si on veut en détruire beaucoup, et faire périr en partic leur progéniture naissante. Néanmoins il est des momens et des jours pour cela plus favorables les uns que les autres; cet animal n'aime ni la pluie, ni le froid, ni même la chaleur; les jours que la température est froide ou variable ne sont pas avantageux pour chasser les taupes; on peut en trouver quelques-unes à l'ouvrage dans les endroits abrités, comme dans un jardin clos, près des buissons ét des haies, ou contre un mur, mais rarement en plein champ; voilà pourquoi le taupier ne doit se mettre en campagne, pour faire la guerre à ces animaux, que lorsque le temps est doux et serein, que la terre n'est point trempée par la pluie, et que le soleil frappe directement les lieux qu'il va exploiter; alors il est sûr de trouver ces animaux à l'ouvrage, et s'il est adroit, il peut compter sur leur complète destruction ; cependant il y a encore certaines heures du jour plus arantageuses les unes que les autres pour surprendre les taupes. Nous avons dit qu'elles avaient des retours réguliers pour leur travail, et qu'elles se mettaient à l'ouvrage trois fois dans la journée : la première fois, quand le soleil a enlevé la rosée; la seconde fois à midi, et la troisième fois à la chute du jour ; il est essentiel de choisir ces momens pour opéror, d'autant plus qu'ils sont fort courts, car le matin, l'occupation de la taupe est d'à-peu-près une heure, et celles du milieu du jour et du soir ne vont pas à vingt minutes chaque.

Le taupier sachant lo licu, l'époque et l'instant favora- 
bles pour prendre les taupes, il ac lui reste qu'à bien étudier la forme de la demeure de ces animaux et la marche de leurs travaux, pour mettre à profit toutes les ressources de son art.

La demeure des tanpes est quelquefois un trou de forme sphérique, fig. $2^{\mathrm{e}}$, quelquefois un boyau long et large, creusé à deux pieds sous terre, $f g . \mathbf{3}^{\mathrm{e}}$; à partir de ce gîte souterrain, la taupe creuse horizontalement et ouvre plusieurs galeries, plus ou moins longues, dans des directions différentes, qui communiquent entr'elles par des boyaux et des coupures intermédiaires ; un tuyau, souvent perpendiculaire, quelquefois incliné, dont le diamètre est celui de l'animal, existe au-dessus des galeries; il est apparent à la surface du sol, et son ouverture est un indice d'évasion; ce tuyau, dont la profondeur ordinaire est de dix-huit pouces, sert à la taupe pour s'enfoncer dans sa demeure lorsque la crainte la saisit. Le taupier habile sait apprécier les différentes modifications que les taupes donnent à leur ouvrage : par exemple, quand la taupe cherche sa nourriture, elle ne fouille gu'en traçant à la superficie du sol, ou dans une terre douce. Une trace est un déchirement de terre ou de gazon : une taupe qui trace va vite en affaire; on prétend que, dans une heure, elle fait trente toises de chemin; lorsque la taupe est en chaleur, elle creuse des traces de trois à quatre pouces de large, et d'un à deux pouces d'élévation; la jeune taupe trace en sinuosité; le mâle forme en traçant de longues allées droites de cinquante à soirante toises; la femelle ne fait pas dix toises en ligne droite. C'est lorsque la taupe se cantonne qu'elle fait le 
plus ordinairement des galeries, des gîtes, des nids, et enfin tout ce qui est nécessaire à son existence locale; on dit (qu'une taupe se cantorme, lorsqu'elle se forme un domicile d'hiver; les nids et les gîtes des taupes sont presque toujours à l'endroit le plus profond et le plus fortifié du domicile, et la galerie dans laquelle elles s'établissent est plus grande et plus élevée que les autres, elle aboutit à deux taupinières opposées. Les galeries des taupes sont plus ou moins grandes, selon la force et le sexe de l'animal; elles sont plus ou moins profondes, selon l'état thermométrique des saisons, par une raison toute naturelle; la température a une influence directe sur les vers et les insectes qui font la principale nourriture des taupes; la douceur ou la rigueur des temps les font monter ou descendre, les taupes ne manquent pas de les suivre. Selon la nature du sol, elles sont aussi plus ou moins superficielles; ainsi, dit M. Demarest, quand le terrain est sablonneux, les racines sont peu profondes, et les insectes s'enfoncent peu; les galeries des taupes rasent, pour ainsi dire, la surface du terrain, et font elles-mêmes une saillie au-dessus; au contraire, quand le terrain est à la fois gras et léger, les travaux sont profonds et pressés avec une activité telle, qu'ils occupent un développement quadruple des premiers. Dans tous les cas, on distingue facilement les galeries faites par un mâle de celles qui sont faites par une femelle. Le mâle, plus robuste, travaille avec plus d'ardeur, et ses galeries et sa demeure sont plus grandes et plus spacieuses; celles de la femelle sont plus petites et plus nomhreuses; la jeune taupe ne creuse pas profondément, elle ne fait que tracer de petites allées à fleur de terre, qui n'ont ni forme, ni régularité. 
Enfin, on peut considérer le domicile de la taupe comme un vaste palais souterrain que partagent deux longs et larges corridors, à côté desquels se trouvent placées, les unes auprès des autres, de petites lignes droites ou sinueuses, qui ont des issues sur le sol, et qu'on a nommées galeries ou boyaux, selon leur grandeur ou leur direction; les corridors, ce sont les passages ou les routes que les taupes fréquentent continuellcment pour visiter leurs galeries, ou pour aller fouiller la surface du sol; ils sont terminés à leur extrémité par une ouverture ou tuyau perpendiculaire qu'un dôme de terre entoure. Souvent la taupe se repose, c'est-à-dire fait son gîte dans une de ces galeries; souvent elle le fait dans un trou qu'elle s'est formé. Le domicile de la taupe, ou plutôt le lieu où elle se cantonne, est toujours artistement construit, mais il n'est pas d'une régularité géométrique; les obstacles qu'elle rencontre dans la terre la font plus ou moins dévier du plan qu'elle se tracerait si elle était sur un terrain également doux et facile à labourer' une filière d'eau, une couche de marne, un roclier, une forte racine, sont pour cet animal des obstacles qu'il ne sait franchir que par de longs détours; c'est par des signes extérieurs qu'on peut apprécier justement l'ensemble et l'étendue de tous ces travaux souterrains ; c'est pourquoi il est essentiellement nécessaire au taupier de saroir bien distinguer les différentes taupinières qu'il trouve dans le champ où il va chasser.

Cadet de Vaux, dans son ouvrage sur les taupes, a traité cette partie d'une manière très sarante; il distingue les taupinières de passage et celles de cantonnement; les tau- 
pinières d'cintréc , celles de sortie, etc.; ii montre les différences qui existent entre les unes et les autres, et les arantages qu'il y a d'attaquer plutôt celles-ci que celles-là. Nous sommes loin d'improuver le travail de cet écrivain philanthrope, mais nous croyons que la description que les ourrages d'agriculture donnent des différentes taupinières, suffit pour instruire le taupier sur cette partie importante de soil art, et nous nous en tiendrons à cette description.

Ainsi, lorsqu'un taupier entre dans un héritage pour tendré ses piéges, il doit explorer les lieux et savoir le nombre de taupinières qu'ils contiennent, et distinguer celles qui sont fréquentées de celles qui ne le sont pas. Les taupinières, dont la terre est fraîchement remuée, annoncent l'existence des taupes; partout où l'on trouve de ces taupinières, on peut à coup sûr dresser ses piéges et guetter l'animal, à moins que la taupinière ne soit percée à son centre par un grand trou; alors c'est une preure que la taupe l'a quittée tout récemment. Les taupinières dont la terre émiettée est sèche et brisée, et qui sont percées par un trou droit et profond, $f g$. se $^{\mathrm{e}}$, ne recèlent point de taupes; on peut, lorsqu'on en trouve de pareilles, les combler ou en affaisser la terre, afin d'empêcher les loirs, les belettes, etc., des'y réfugier. Une taupinière haute, grosse et isolée, indique la présence d'une taupe mâle; plusieurs de ce genre, renfermées dans un espace de terrain, peuvent être l'ouvrage d'un seul animal, mais souvent elles en con-

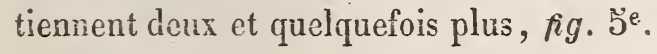

Les taupinières qui sont plus petites et moins élevées, 
annoncent la demeure des femelles, $\mathrm{fg} .6^{\mathrm{e}}$; celles qui sont en zig-zag, irrégulières peu élevées, décèlent la présence des jeunes taupes, fig. $7^{\mathrm{e}}$; ainsi, lorsque le taupier connaît bien l'ouvrage des taupes et la marche périodique de leurs travaux, il n'a qu'à les surprendre dans l'endroit de leur domicile où elles se trouvent, et rompre avec la bêche ou la houe le boyau de communication qui la sépart du centre de ses galeries; par-là il l'emprisonne entre deux points très rapprochés, et il €st sûr d'en être bientôt le maître. Arec les connaissances préliminaires que nous venors d'exposer, celui qui voudra faire la chasse aux taupes pourra aller tendre ses piéges, ou bien répandre ses appâts empoisonnés, et il sera sûr de réussir. 
DES DIVERS MOYGNS DE FAIRE LA CEASSE AUZ TAUPES.

Hoyens de faire fuir les Taupes du lieu où elles se sont étab̉lies.

Si j'indique les moyens qu'il faut employer pour chasser les taupes d'un champ, ce n'est pas pour inviter les cultivateurs à les suivre; je n'admeitrai jamais ce principe d'égoïsme qui nous fait sacrifier les intérêts d'autrui pour les nôtres, et, dans ce cas, ce principe est trop dangercux, même pour celui qui le met en pratique, pour se permettrè de le tolérer; en envoyant chez le voisin ce qui peut lui ıuire, le voisin naturellement cn agira de même à votre égard, ei par cet échange de mauvais services, on laissera exister long-temps ce qui incommode et nuit à l'un et à l'autre.

Je veux donc montrer dans ce chapitre, que les moyens infaillibles que l'on emploie pour chasser les taupes, ofient rarement des résultats heureux; que ces anmaux ne font que changer un moment de domicile, lorsque la terre d'où on les chasse est douce, légère et abondarmment pourva de vers ou de larves, et que, pareils aux oiseaux de passaçe, ils reviennent dans la contrée qui leur plait, apròs 


\section{$-34-$}

que les causes qui les avaient éloignés n'existent plus. Les liorticulteurs du siècle passé ont inventé beaucoup de recettes et donné beaucoup de procédés pour chasser les taupes d'un héritage; les uns recommandent de ficher dans leurs galeries de petits bâtons de sureau, ou de jeter dans leurs trous de petites branches de saule, du chanvre vert, de la poirée, de l'ognon, de la fiente de cochon, etc.; les autres donnent comme infaillibles les écrevisses pourries, répandies autour des taupinières, et les noix bouillies avec du sel et du sulfate de fer. Paxanus conseille de placer dans leurs trous un petit vase étroit et solide, et d'y mettre de la paille avec de la résine de cèdre, ou, selon Paladius, de la cire; enfin on chasse les taupes d'un champ avec de l'acide sulfureux, avec tous les gaz qui ont une odeur forte et pénétrante, avec l'ean, la fumée de bois ou celle des herbes aromatiques; pour cela, on enlève les taupinières, on fait des coupures à travers les boyaux et les galeries, et l'on brûle, dans ces espèces de tranchées, le soufre, la plante ou le bois dont on veut se servir, en ayant la précaution de diriger la vapeur ou la fumée dans l'intérieur des boyaux. On complète l'opération en poussant la matière inflammable en ignition, et à moitié consumée, dans les boyaux, et en fermant les ouvertures : si le domicile de la taupe est peu profond, souvent ces moyens suffisent pour étouffer l'animal; mais on ne doit pas compter là-dessus, on doit s'attendre seulement que lorsque la taupe ira visite: ses galeries, elle se trouvera surprise par une odeur suffocante, et qu'elle cherchera à s'échapper : c'est ce qui arrive toujours. Un taupier de Paris, nommé Calme, imagina, il y a une quarantaine d'années, un appareil pour introduire 
dans les réduits des taupes les fumigations de soufre ef de tabac, afin d'étouffer ces animaux ou de les faire fuir; voici comment il est construit : à la clayère d'un soufflet à deux âmes, et un peu fort, est ajusté un fourneau de cuivre ovale, qui reçoit des charbons allumés, semés par-dessus de soufre ou de tabac à fumer; l'on visse, à l'extrémité de ce petit fourncau, un bout de tuyau de forme cônique, qui sert à diriger, dans le réduit de l'animal, la fumée qu'entretient le jeu du soufflet. J'ai fait différentes épreuves arec les recettes indiquées par les auteurs, elles m'ont toutes réussi, je veux dire que les taupes ont fui de la pièce de terre où je les ai attaquées; mais elles sont revenues le printemps suivant : je vais citer un exemple pour prouver cette vérité. Dans un pré qui contenait cinquante-six taupinières, et dans lequel je soupçonnais la présence de douze à quinze taupes, je fis l'expérience suivante : j'enlevai avec une bêche toutes lcs taupinières jusqu'à leur tuyau de communication, après m'ètre assuré de la direction du passage souterrain; je fis quelques incisions à deux ou trois pieds des taupinières et sur le passage de la taupe ; j'enlevai soigneusement la terre que j'avais fait tomber en crcusant, "afin de bien mettre à découvert les trous des boyaux et des galeries que j'arais incisés, et entre ces coupures je fis brûler des f́cuilles de noyer et des pelures vertes de noix que je trouvai sous ma main ; j'eus soin, pendant la combustion, de bourrer l'intérieur des trous avec la matière inflammable en ignition, et de fermer les ouvertures; cette opération dura plusieurs heures; le lendemain matin je visitai le pré, ct je trouvai sur le sol, par-ci par-là, non pas les taupinières ouvertes, parce que je les avais fermées avec des 
feuilles de noyer à demi-consumées, mais des trous parfaitement ronds et sans monticules; je soupçonnai alors que ces animalx avaient fait dans la nuit ces nouvelles issues, et qu'ils s'en étaient échappés; et en effet, de toute la saison je ne vis dans ce pré ni taupes, ni ourrage qui pût indiquer leur présence ; mais dans les pièces de terre voisines du pré, quelques taupinières furent élevées; le taupier de la ferme, qui vint alors faire sa tournée, tua une partie des taupes qui les avaient formées, et il m’assura, lorsque je lui fis le détail de mon opération, que l'année suivante le pré serait de noureau infecté de taupes, qu'elles feraient de nouvelles taupinières et de nouveaux souterrains; je vis alors que je n'avais fait que reculer le mal pour le rendre plus grave, car ce que me prédit le taupier fut à la lettre, et le printemps suivant les taupes vinrent prendre résidence dans le pré, et y firent de nouveaux ravages; cet exemple doit suffire pour ôter aux cultivateurs l'envie de faire fuir les taupes de leur héritage, et ils doivent se convaincre que le meilleur moyen de s'en débarrasser ou de les rendre moins nombreuses, c'est d'en faire périr le plus que l'on peut.

Lorsqu'on veut faire déloger une taupe de son domicile par le moyen de l'eau, on s'assure du nombre des taupinières qu'elle a faites, on les enlève, on creuse un peu avant dans la terre pour metire bien à découvert leurs boyaux, et on verse dans chacun une quantité d'eau assez grande pour qu'elle pénètre jusque dans l'intérieur du domicile; ce mojen ne rénssit que lorsque le róduit de la taupe est per profond, et qu'il u'a par de nombreuses issues. 
Des substatices végétales et minérales que l'on emploie pour faire périr les taupes; de la manière de les mettre on usage, et de l'opinion que l'on doit avoir sur los appats empoisonnés.

Quelque bien établie que soit, dans les campagnes, l'idée qu'on peut détruire beaucoup de tâupes avec les substances végétales vénéneuses, je puis affrmer qu'elle est loin d'être fondée : sans doute la ciguë, le stramonium, la belladonne, le tabac feraient périr les taupes, si ces animaux pouraient s'en nourrir ou se laisser surprendre par des appats imprégnés du suc do ces plantes; mais il est prouvé que, loin d'en faire usage, elles les ont en horreur. Sous quelle forme que soient les poisons végétaux, ils portent avec eux une odeur forte et nauséabonde qui inspire le dégoût à presưue tous les quadrupèdes; les insectivores et les herbivores sont comme les hommes, ils savent choisir leurs alimens, et ceux qui sont les plus frais et qui leur plaisent le mieux, sont ceux qu'ils préfèrent. Les taupes sunt très délicates, tout aliment qui n'est pas fr'ars et naturel ne leur plaîl pas; elles sont aussi très sabres, peu de chose contente leur appétit, et la variété des plats n'est pas ce qu'elles recherchent ; tout leur apprêt gastronomique ne se compose que de quelques vers et de quelques racines qu'elles trouvent abondamment dans l'intérieur de la terre : de-là vient l'indifférence qu'elles montrent pour tous les appâts qu on leur donne. Je soutien donc que les plantes vénénenses et narrotiqnues ne sont pas 
propres à faire périr les taupes, parce que ces animaux ne les mangent pas parce que leur incorporation dans l'aliment qu'on veut empoisonner suffit pour les leur faire dédaigner; je ne crois pas non plus que les noix bouillies dans unc forte lessive soient propres à cet usage, car la décoction que la noix a subie dans un vélicule alcalin, lui donne un goût désagréable et une odeur repoussante, en saponisant sa pariie huileuse; plusieurs expériences faites à ce sujet m'ont convaincu de la vérité de ce que j’avance. Le secret d'empoisonner les taupes par les noix bouillies dans la lessive, a été donné par les nommés Mitchet et Habattre, habitans d'Ostubac, dans la BasseBretagne, comme un des meilleurs moyens pour les détruire; ils prétendent s'êire exercés pendant deux ans à faire périr les taupes qui désolaient leurs terres, et qu’ils yont parfaitement réussi. Il est étonnant qu'en Bretagne les taupes aient des goûts différens de celles des environs de Paris. J'ai fait plus d'un essai, tant avec les noix boullies qu'avec d'autres poisons végétaux, et je n'ai jamais eu lieu d'en être satisfait : voici ce que je peax affrmer. J'ai choisi cinq taupinières séparées les unes des autres, et contenant chacune une taupe mâle, je les ai enlevées avec la hone, pour bien m'assurer de quel côté était le boyau communicateur des galeries; j’ai fait, à deux pieds de la taupinière, deux incisions à ce boyau, et j'ai placé aux deux extrémités de chaque coupure, savoir : à la première taupinière, de la ciguë, du stramonium et de la racine d'cllébore fraîchement cueillis; à la deuxième, j’ai mis des squammes fraîches d'ognons de colchique, des pellicules blanches de porrcau et de racine du Sceau de Salomon, saupoudrées 
légèrement de ciguë et de jusquiame; ì la troisième, jai mis des vers de terre tout virans et des chenilles, et j'ai répandu, dans l'intérieur diı boyau, de la poudiè déjà citéc, afin que ces animaux puissent s'en courrir en rampant dessus; à la quatrième, j’ai mis des larves de hannetons et de chenilles, entre l'ćpiderme desquellex j'avais introduit de la même poudre; dans la cinquième, j'ai mis des noix qui araient été bouillies dans une forte lessive, et des boulettes faites avec des vers et des larres imprégnés du suc de belladonne; j'ai laissé ces appâts pendant cinq jours dans l'intérieur du boyau, et, au bout de ce terme, je les ai trourés intacts et tels que je les avais mis, excepté les vers qui s'étaient enfuis. Deux taupes seulement avaient abandonné leur domicile. J'ai plus d'une fois mis d'autres appâts qui cachaient d'autres plantes vénéneuses, telles que le tithymale et la chélidoine, etc., etc., et j’ai presque toujours eu le même résultat. Je dis presque, car une fois arant amorcé les trous de deux taupes avec un appât dont la recette sc trouve dans le Dictionnaire économique de Chomel, rédigé par Delamarre, les taupes le mangèrent, et une d'elles mourut dans son réduit. Voici la recette : prenez de la racine d'ellébore ou de l'écorce de cynocrambe (mercuriale sauvage), ou d'apocyn, pilée et tamisée, et l'ayant bicn arroséc de farine d'orge et d'œuif, détrempez, pétrissez le tout avec du vin et du lait, compez-le en petits morccaux, et jetez-les dans les trous. J'attribue au goût particulier que les taupes ont pour l'orge et les cuufs, la réussite de cet appât.

Voici une autre recette rịui, dans son temps, a été don- 
née conme infaillible : prenez autant de noix qu'il y a de trous de taupes, avec une poignée de ciguë, et faites bouillir le tout une heure et demie dans de l'eau; faites-en des - spèces de boulettes, ou si la pâte est trop liquide, mettezen sur un morceau d'ardoise dans les trous. Tous ces appâts, je le répète, restent le plus souvent dans les trous des tanpes sans être touchés; on a plus d'avantage et plus de succès d'apprêter les appâts pour les taupes avec des poisons minéraux. Saupoudrer légèrement d'arsénic le blanc d'un porreau frais, un ognon de colchique, ou bien des vers de terre, ou quelques grosses larves de hannetons, placez-les aux extrémités des coupures que vous avez faites aux galeries, et l'animal viendra de temps en temps se prendre; mais comme nous l'avons fait observer, la taupe est délicate, elle préférera tonjours les alimens naturels qu'elle trouve en fouillant la terre, à ceux que lui apprête une main étrangère. Les expériences que j’ai faites à ce sujet m’ont couvaincu que les substances végétales vénéneuses sont d'un très petit secours pour détruire les taupes, que l'on réussit mieux avec les poisons minéranx; mais qu'il vaut mieux employer, pour les détruire, les moyens naturels et les piéges.

\section{Méthode naturelle de prendre les taupes.}

J'appelle ainsi le moyen de surprendre la taupe dans son travail, et de s'en rendre maitre, en lui coupant tout moyen de communication avec son réduit, par un coup de bêche ou de houe que l'on donne à un demi-pied de terre dans le boyau qu'elle établit. 
Je sais les éloges mérités que l'on donne à la manière de prendre les taupes de Lafaille et de Lecourt; j'apprécie les connaissances pratiques de d'Aurignac, que M. Dralet nous a fait connaître, et je me dispose à consacrer un article à chacune de ces méthodes; mais, je dois le dire, la manière simple et facile dont les jardiniers ct les gens de la campagne prennent les taupes, si elle n'est point la meilleure, doit être la plus généralement suivie; car, indépendamment qu'elle est bonne, elle abrège le temps, et n'entraîne ni embarras ni dépenses; cette méthode, du reste, est la plus ancienne de toutes; le cultivateur, en tout temps, s'est occupé à prendre ou à chasser de son héritage les taupes, lorsqu'elles lui étaient trop incommodes, et il ne s'est jamais servi de piéges ni de filets; encore aujourd'hui les jardiniers et les cultivateurs ne font usage que de leur bêche ou de leur houe pour détruire les taupes, et les paysans du midi de la France sont si habiles à cette chasse, que sur douze on quinze taupes qui habitent dans un pré, ils vont gager de n'en pas manquer une. Comment font-ils? me diront ceux qui ne savent se servir que de piéges et de filets. Ils font à-peu-près comme quand le chat guette la souris : quand ils ont aperçu, dans leur jardin ou dans leur terre, une taupinière fraìchement élevée qui récèle une taups, ils prennent l'heure où cet animal travaille, ils vont se blottire tout à côté de sa nouvelle fortification, et au moindre mouvement de l'ouvrier, ils lancent un coup de bêche à un demi-pied de terre, du côté opposé où ils ont aperçu que la taupe rejetait son déblai, et ils trouvent ordinairement dans la motte qu'ils soulèvent, l'animal qui ne peut leur échapper; si, comme cela leur arrive quelquefois, il chasseur tatPIER. 
leur échappe, ils abaissent la taupinière et ils attendent is taupe à son retour au travail; souvent ils n'ont pas la peine d'attendre long-temps, car elle vient tout aussitôt qu'elle s'aperçoit qu'on a détruit sa taupinière.

Si la demeure de la taupe qu'ils poursuivent a plusieurs taupinières, ils les affaissent toutes en piétinant dessus, et ils restent en sentinelle. Les taupes, pour ne pas aimer ni le grand jour ni le grand air, n'ont pas moins besoin, pour vivre, de la présence de l'air atmosphérique qui leur arrive du trou de la taupinière par les nombreuses galeries; lorsque cet air est intercepté, elles ne manquent pas de se diligenter pour lui ouvrir une issue, et lorsqu'on ferme leurs taupinières, on les voit bientòt paraître et pousser la terre. D'un autre côté, la taupe est amie de la iiberté : du moment qu'on ubstrue une des routes qu'elle s'est tracées, elie n'a rien de plus empressé que de la réparer; cette constante manière d'opérer rend facile la prise de ces animaux, car il suffit de faire des coupures à leurs galeries ou de détruire leurs taupinières, pour les voir accourir réparer le dommage, et alors il est d'autant plus facile de s'en rendre maître, qu'elles sont plus ardentes au travail, et, par conséquent, moins méfiantes. Ainsi, on peut prendre les taupes par la méthode naturelle, au moment de leur traval, en comblant leurs taupinières ou en faisant des coupures à leurs galeries: dans l'un ou dans l'autre cas, il suffit de les guetter en silence, et de leur couper toute communication avec leur souterrain. On serait presque toujours sûr de prendre une taupe qui travaille à un assemblage de taupinières, si on voulait prendre la peine de les. 
cnlever toutes et de découvrir tous les boyaux; mais ce procédé est trop long et trop pénible ; cependant on le met quelquefois en pratique pour détruire les nids des taupes : lorsqu'on a découvert un de ces nids à l'époque du part, plusieurs hommes, munis de bêches, se placent autour de ce gîte, et à un signal domné ils coupent toutes les galeries qui communiquent arec le fojer paternel, ensuite ils l'attaquent et détruisent la taupe arec ses petits.

De la manière de prendre les taupes avec des chiens.

Childrey, dans son Histoire des singularités d'Écosse, dit qu'il y a, auprès de Portsmouth, une race de petits chiens dont on se sert, en ce pays, pour faire la guerre aux taupes; je puis assurer que presque toutes les espèces de chiens peuvent être employées à cet usage; il suffit de les dresser comme il convient. J'avais, lorsque j'llabitais le Dauphiné, un chien noir, le ventre blanc, à poil ras et luisant, de moyenne taille, qui avait un talent singulier pour prendre les taupes, et je puis dire qu“il réussissait beaucoup mieux que les taupiers de la contrée. Le matin, à l'heure que les taupes travaillent, je l'amenais dans un pré où je savais trouver des taupinières fraîches; arrivé sur le pré, lui-même reconnaissait les plus propres à être altaquées, il se couchait à côté, et attendait que la terre remuàt assez fort pour lui annoncer la présence de la taupe; daus le moment il sautait sur le tas de terre, et avee ses pattes et son museau il enlevait la forre et l'ani- 
mal; ayant une fois réussi, il fallait qu'il en cût plusieurs, et je lui en ai vn prendre six et huit certains jours, selon les torrains et la température; cependant il n'en a jamais mangé, mais il les trait et les lâchait seulement quand elles ne bougeaient plus.

Pour dresser les chiens à prendre les taupes, il faut les prendre fort jeunes; on les amène pendant la saison qu'elles travaillent le plus, dans une pièce de terre où se trouvent beaucoup de taupinières, on lui fait parcourir toute la pièce de terre deux ou trois fois, pour lui en faire prendre le flair, et après on le laisse en arrêt auprès d'une taupinière fraîche et isolée, que l'on a préalablement affaissée; la taupe ne manque pas de venir réparer sa fortification, le chien qui la guette, se jette sur elle au moment qu'il l'aperçoit; dans les premiers jours il manque ordinairement sa proie, mais par la suite il devient plus adroit et plus avisé, et sans s'amuser à fixer le centre de la taupinière par où sortent les miettes de terre que la taupe rejette, il pousse la taupinière avec son museau et fait sauter la taupe sur le pré.

Des armes à feu, comme moyen de détruire les taupes.

Je ne crois pas que les armes à feu soient d'une grande utilité pour détruire les taupes, et ce qui me fait penser ainsi, ce sont les raisons que plusieurs avancent pour les admettre comme un moyen de destruction : M. Dralet, dans un article intitulé : Supplément $\dot{a}$ l'Art du Taupior, 
inséré dans lia feuille du Cultivateur de l'an 6, s'exprime ainsi à ce sujet: Lorsque la taupe forme des taupinières dans un carreau de jardin, ou dans tout autre terrain que l'on craint d'endommager par des incisions, on a recours aux armes à feu; pour ne pas perdre son temps inutilement, il faut guetter la taupe aux heures indiquées, charger un fusil à petit plomb, et tirer presque à bout portant; par ce moyen, si l'animal échappe au plomb, il peut être étouffé par la fumée; mais il faut avoir la précaution de diriger. votre coup vers l'endroit d'où la taupe apporte la terre; pour connaitre cet endroit, enlevez d'abord avec la bêche la taupinière, et creusez-la jusqu'à ce que vous trouriez les boyaux qui y aboutissent; la taupe viendra réparer ces dégâts, vous verrez de quel côtéelle apporte la terre à l'endroit endommagé, et c'est vers ce côté que vous devez diriger le coup. Si les armes à feu ne sont utiles que dans le cas dont parle M. Dralet, il est en fait qu'elles ne remplissent pas le but qu'on se propose, puisque, en eulevant la taupinic̀re et en creusant jusques aix boyaux communicateurs, on fait le dommage que l'on voulait éviter; d'un autre côté, si la taupe ne sort pas de terre pendant son travail, très certainement on la mancquera, et si le plomb ne peut l'atteindre, à coup sûr la fumée ne l'atteindra pas mieux; et puis enlever la taupinière, la creuser jusques aux boyaux, attendre que la taupe vienne réparer le dommage, tout cela demande du temps et de la peine, et le procédé, à mon avis, est loin de valoir la méthode naturelle, qui se borne à donner un conp de houe à la gorgge du boyau où la taupe souffle. 
Manière de prendre les taupes avec de petits faisceaux de bois épineux.

On sait que la taupe, blessée au sang, meurt presque toujours; cela a fait naître l'idée de mettre dans les galeries d'une taupe, de petits faisceaux de bois épineux, afin que l'animal, obligé de passer à travers, puisse se blesser et mourir : voici comment s'exprime l'auteur de ce procédé, dans la Bibliothèque physico-économique : "Il faut fouiller un peu la terre sous ces petites éminences fraîchement remuécs, connues sous le nom de taupinières; on y trouvera une ligne de conduite horizontale; il faut bien nettoyer les deux avenues et placer au fond de chacune un petit faisceau de ronces choisies, dont les épines soient fortes et bien aiguës, de la longueur de quatre à cinq pouces, et de grosseur suffisante pour remplir exactement la capacité de ce boyau souterrain, après quoi l'on remet de la terre que l'on foule un peu avec le pied; la taupe, en suivant la route qu'elle s'est tracée, vient s'y piquer et périt.

On pourrait y substituer de petits bâtonnets garnis de pointes de fer par leurs deux bouts, ce qui serait plus dispendieux, mais plus certain. Dans l'ouvrage de M. Mortimer, intitulé : Agriculture complète, ou l'Art d'améliorer les Terres, il est fait mention d'une taupière à-peu-près semblable. On se sert, pour cette sorie de pićge, ou de l'églanticr, ou de l'épine blanche; les labitans du Velai ne prennent les taupes que de cotte manière. 
Manière de prendre les tanipes avec un pot de terre.

Ce moyen nous a été conservé par Olivier de Serre, et tous les auteurs qui ont parlé sur l'art de prendre les taupes l'ont répété : si je le rappelle à mon tour, c'est que je crois qu'on peut le mettre avantageusement en usage, en mars et en avril, époque où les taupes se recherchent pour s'accoupler.

Pour préparer le piége comme il convient, on se munit d'un pot de terre vernissé à l'intérieur, ou plus étroit en haut qu'en bas, et assez profond, fig. 11 et 12, afin que l'animal u'en puisse sortir; on place dans le fond une taupe femelle, ct on ra l'enfoncer dans un pré jusquu'à fleur de terre; cette opération se fait le soir; on doit chercher, pour placer le pot, un pré ou une pièce de terre où il y ait leaucoup de trous de taupes; dans la nuit, la femelle, qui se trouve emprisonnée, ne manque pas de crier et d'appeler à elle les taupes ses voisines, qui , ordinairement, accourent et se précipitent dans le pot, où elles sont obligées de rester.

On peut les attirer aussi, en metlant des écrevisscs vivantes dans le pot. C'est ainsi qu'on prend les taupes dans plusieurs cantons de la Suisse.

Un propriétaire, qui arait un champ que les taupes désolaient, s'avisa de faire des trous on terre pour y placer des pots un peu profonds, comme, par exemple, une cru- 


\section{$-48-$}

che : il plaça cette cruche entre quatre routes que les taupes avaient pratiquées; il couvrit le dessus arec un pan de gazon pris dans un pré, observant de ne pas boucher les routes, et ayant le soin d'enterrer la cruche un peu andessous des galeries. Au bout de cinq jours il leva le gazon pour regarder dans la cruche, il y arait vingt-une taupes dedans, toutes noyées, parce qu'il avait mis au fond environ un demi-pied d'eau. Dans quinze jours de temps il a détruit tous ces animaux, et le voilà tranquille sur le produit de son jardin. Cette même personne assure que le prince de Salm en avait une infinité dons ses jardins et dans ses prairies, et qu'il a détruit toute cette peuplade par le même moyen.

Il faut croire que ces animaux passent et repassent souvent par les routes souterraines qu'ils se pratiquent; croyant aller de plein pied, ils s'engloutissent dans le piége.

Manière de prendre les taupes avec des hamegors.

Ayez un hameçon attaché au bout d'une ficelle mincc et solide, a.norcez-le arec des vers vivans ou avec quelque larve de chenille; introduisez cet appât dans l'intérieur du boyau où passe la taupe, attachez en dehors, à un coin de. bois ou à une pierre, l'autre bout de la ficelle, et si l'animal passe et qu'il sente le ver virant, il mordra à l'hameçon et s'y prendra.

On place ordinairement cette sorte d'appât le soir, après le soleil cauché, et on va le visiter le matin, entre onze 
heures et midi; si à celte époque la taupe n'est pas prise, il faut amorcer avec de nouveaux vers. Lorsqu'une taupe a fait, antour de son domicile, plusieurs taupinières, on place dans le boyau de chacune d'elles un hameçon amorcé, c'est le moyen de la prendre plus vite.

Moyen de prendre les taupes dans quelques contrées d'Allemagne.

Il y a dans certaines contrées d'Allemagne, des taupiers si habiles, qu'ils prennent souvent, par jour, plus de cent taupes, par un moyen très simple. Ils savent que, de chaque taupinière, descendent deux ou trois conduits ; ils cherchent ces canaux, et fichent dans chacun, à une petite distance du centre de la taupinière, un bàton ( ou une baguette) écorcé jusqu'au blanc, de la longueur environ de deux pieds à deux pieds et demi, en observant bien la direction de chaque conduit souterrain; il y a, par conséquent, antour de chaque taupinière, ordinairement deux à trois de ces baguettes; pontr mieux découvrir les conduits, ils enlèvent la terre des taupinières et la remettent ensuite. Après avoir ainsi fiché leurs bâtons dans une vingtaine ou trentaine de taupinières, ils se placent au milieu avec une bêche peu large, ayant l'ex trémité inférieure droite et tranchante; dès qu'ils observent le mouvement d'un bâton, ils accourent à pas légers et enfoncent vite leur bêche à environ un pied de distance du bâton, au-delà de la taupinière; ils ferment par-là la retraite à la taupe, et ils les retirent ordinairement virantes. 
Aux hcures de la journée où les taupes travaillent, à six heures du matin, à midi et à six heures du soir, un taupier peut à peine courir d'une prise à l'autre, aussi promptement que ses bâtons remuent, et il n'est pas extraordinaire de leur voir prendre douze à vingt taupes de suite, si le circuit qu'ils ont choisi pour leur chasse est un peu grand.

Moyen de prendre les taupes avec de l'eau.

Aux environs de Langres et de Chaumont, on se sert de l'eau pour détruire les taupes, et voici comment on s'y prend : on va dans un champ fréquenté par les taupes, aux heures où ces animaux sont à l'ouvrage; on verse, par le tuyau d'une taupinière fraîchement élevée, assez d'eau pour humecter tout l'intérieur du domicile de l'animal; celui-ci, se sentant mouillé, se hâte de quitter son réduit, et on le saisit à sa sortie.

Ce procédé n'est pas infaillible, comme on peut le penser, cependant il réussit toujours sur une taupe qui n'a pour asile qu'une taupinière peu profonde.

Héthode de prendre les taupes, par d'Aurignac, publice par M. Dralet.

La méthode de prendre les taupes, de d'Aurignae, est fondée sur l'habitude constante et naturelle que ces animaux ont de venir réparer tout aussitòt le dommage que l'on fait à leurs habitations. Les moyens qu'on emploie sont 
les coupures ou des incisions faites à propos aux boyaur souterrains; selon le nombre des taupinières et la direction des travaux que l'animal entreprend; de la paille, du papier, un peu d'eau et une houe, sont nécessaires pour faire, arec succès, cette espèce de chasse.

La méthode de d'Aurignac ust une des meilleures que l'agriculture possède, et tous les écrivains agronomes en sont convenus; cependant, il faut le dire, cette méthode demande beaucoup de temps et de peine, et elle ne peut être avantageuse que pour ceux qui ont beaucoup de temps à perdre, ou pour les taupiers de profession.

Un jardinier ou un petit cultivateur, qui, d'un clin-d'œil, parcourt tout son héritage et voit tout ce qui s'y passe, préférera toujours la méthode naturelle, ou les moyens faciles que la taupière simple procure, au procédé de d'Aurignac, parce qu'il aura moins de temps et de peine à perdre ; mais, il faut en convenir, dans les grandes propriétés et dans les contrées où il y a beaucoup de taupes, ce proçédé doit être préféré à tout autre, attendu qu'il a l'avantage d'en faire prendre beaucoup à la fois. Mon intention étant de faire connaitre l'ensemble de cette méthode, je ne m'amuserai pas à donner un air de nouveauté à ce chapitre, de calquer mes exemples sur célui de son auteur, car ce qui est à César doit être rendu à Cesar; je suivrai tout simplement la description qu'en fit M. Dralet, dans la feuille du Cultivateur de l'an 6 . Je suppose, dit cet auteur, une pièce de pré, représentée dans la planche $1^{\text {re }}$, fig. $2,5,4, \breve{5}, 6,7,8,9$ et 10 ; j'apercois, $f i g . ~ \check{~}$, une 
taupinière isolée; clle est fraîche, donc elle annonce l'existence d'une taupe; clle est grosse, donc elle a été faite par un mâle. Les deux taupinières, $f g .6$, sont éloignées l'une de l'autre, elles ont aussi été faites par une seule taupe; elles sont petites, done elles appartiennent à une femelle. Les trois taupinières, $f g .9$, sont peu éloignées, elles appartiennent cncore à une seule taupe, qui est mâle, quand ces taupinières sont grosses et fraiches; la taupe $\mathrm{y}$ travaille. Les six tanpinières, fig. 8, pcu éloignées entre elles, appartienment à une seule taupe; elles sont fraîches et petites, donc elles recellent une femelle. Ces traìnasses en ziğ-zag , ou taupinières informes, $f g .7$, sont fraîches, elles appartiennent à une jeune taupe. Ces trois tanpinières, fig. 10 , sont sèches, donc clles ont été abandonnées; la taupinière, fig. 4, cst encore fraîche, mais elle est percée par le haut, donc la taupe qui l'a faite l'a quittée depuis peu; ces observations indiquent qu’il $\mathrm{y}$ a dans ce pré cinq taupes, deux mâles, deux femelles et nne jeunc; les mâles travaillant plus vite, doivent être gुuettés de plus près que les femelles; on doit aussi veiller de près les jeunes, qui, ne faisant qu'efleurer la terre, vont plus vite en besogne.

Voici à-peu-près comme il s'exprime encore :

Lorsçu'une taupe n'a fait qu'un tron, j'enlève d'abord la taupinière avec la terre, et je m'assure si clle n'a pas de communication arec les taupinières voisines; pour y parvenir, je tousse dans l'ouverture que j'ai faite, c'est-à-dire l'ouverture du boyau commencé; j'en approche en mème temps l'oreille; si la taupinière n'a pas de communication, la taupe est pen éloignée; effrayéc par le hruit, je l'en- 
tends s'agiter, et elle ne pent m'échapper. Je découvre le boyau horizontal avec la hone et le suis jusqu'au bout, ou je rencontre la taupe; mais l'animal, connaissant le danger a peut-ètre eu le temps de s'enfoncer en terre, en continuant perpendiculairement le boyau, $f i g . \overline{5}$.

Alors j'ai deux moyens pour le prendre : je creuse ce nouveau boyan, où je rencontre ma proie; ou tien $j$ 'y verse de l'eau, et l'animal s'y présente de lui-même.

Si en toussant je n'ai pas entendu l'animal s'agiter, c'est la preuve qu'il a au moins deux taupinières, et j'opère de la manière suivante : je fais une ouverture entre elles, de plus de neuf pouces, dans la direction du boyau qui communique d'une taupinière à l'autre, je ferme arec un pen de terre les deux extrémités du bovau ; frappée par le grand air etcraignant pour sa sûreté, la taupe vient, quelques instans après, pour réparer le dommage fait à sa galerie souterraine, elle souffle ou pousse de la terre arec ses pattes en dehors; si elle se présente d'un côté, je suis assuré de la trouver entre ce point et la taupinière; si, au contraire, elle se présente de l'autre côté, je suis certain qu'elle est entre ce point et l'autre taupinière. Dans l'mn ou l'autre cas, j'opère comme si ma proie n'arait pour taupinière que celle où elle se trouve arrêtée.

Si ma taupe a trois taupinières, je multiplie les coupures que je fais entre chaque taupinière, la taupe viendra alors souffler d'un côté ou d'un autre, car ses points de communication étant coupés, et l'animal se trourant renfermé dans l'espace intermédiaire d'une taupinière à la coul- 
pure qui en est proche, elle ne tarde pas à manifester sa présence par son agitation et son soufflement; toutefois, jattends en silence qu'elle ait commencé son travail pour m’en rendre plus facilement maître.

Si une taupe a six taupinières, je fais une incision aux deux taupinières de passage, qui sont toujours les plus éloignées du gîte; si la taupe vient souffler à l'un ou l'autre bout, j'opère alors comme dans le cas où il existe trois taupinières.

Lorsque plusieurs taupinières fraîches se trouvent près des vieilles taupinières, il faut d'abord faire des coupures qui interrompent toutes communications entre les unes et les autres, et lorsqu'on est parvenu à connaître l'endroit où la taupe se présente pour souffler, on agit comme dans le premier cas. Si on attaque plusieurs taupes à la fois, il faut être actifet très vigilant, parce que lorsqu'on est occupé à guetter une taupe, une autre peut traverser le boyau que l'on a découvert. Pour s'apercevoir plus facilement de ses mouvemens, on place dans cet endroit un étendard de paille ou de papier, dont l'agitation ou la chute indique la présence de la taupe, à laquelle on rend encore le passage plus difficile par une petite motte de terre placée dans sa galerie.

Moyens analogues à celui de d'Aurignac.

Les moyens dont se servent une partie des jardiniers des contrées du nord-onest de la France, pour détruire ua 
grand nombre de taupes, nous ont paru avoir de l'analogic arec celui que d'Aurignac a établi en Gascogne, et nous les annonçons comme tels. Lorsque dans un héritage se trouve un grand nombre de taupes, un jardinier, suivi de plusieurs manœuvres, se rendent sur les lieux à l'époque et à l'heure qu'elles fouillent avec plus d'activité , c'est-à-dire dans les belles matinées de mars, et ils choisissent encore un jour où le vent est vif et fort; ils commencent à découvrir toutes les taupinières qui décèlent l'existence d'une taupe à demeure, et ils plantent au bord de chacune et la fixent avec un peu de terre, une petite baguette dont le mouvement sert de signal; armés d'un instrument tranchant, ils attendent en silence que les taupes viennent refermer les ouvertures; ces animaux, mis à découvert et incommodés dans leur réduit par un air frais, ne cherchent aussitôt qu'à y remédier; ils rapportent promptement à ces crevasses une nouvelle terre, qui forme un second monticule ( c'est la taupinière oblongue dont parle M. Dralet); leurs travaux sont annoncés par différens coups de tête qui ébranlent la baguette, et que les chasseurs ne perdent pas de vue; au moindre mouvement ils enlèvent, avec l'instrument dont ils sont armés, et la taupe et son ouvrage; plus le vent du nord est vif, plus l'animal boute arec viracitê. L'auteur qui cite cette manière d'attaquer les taupes, et qui est beaucoup plus ancien que M. Dralet, rapporte que lorsque le temps est favorable, on peut prendre plus de cent taupes par jour, et enfin toutes celles qui se trouvent sur un héritage.

Cette méthode, qui se rapproche de la méthode natu- 
relle, est la plus simple de toutes, et on doit y donner la préférence.

Piége des anciens pour prendre les taupes.

Les anciens se servaient d'un piége arec un nœud coulant de fil de fer, qu'ils avaient soin de placer dans le passage le plus fréquenté du domicile de la taupe; ce piége est encore en usage en France, en Allemagne et dans une partie de la Suisse, et comme il réussit assez bien, il n'est pas indifférent de le faire connaître. Sur un bàton droit on pose une traverse représentant le fléau d'une balance, $e^{t}$ qui en produit l'effet; à l'un des bouts de la traverse est attaché un poids assez fort, l'autre est muni de deux ficelles, dont l'une a à son extrémité un lacs de laiton à nœud coulant, et à l'autre est suspendu un petit morceau de bois de dix à douze pouces de largeur et d'un ponce d'épaisseur; pour tendre ce piége, on met à décourert la route de passage de la taupe, et à une légère distance de cette incision on fait entrer, par une seconde incision faite perpendiculairement à la terre, directement au-dessus du conduit, le lacs bien ourert, que l'on dispose selon la dimension du passage qu'on attaque; on bouche ensuite cette ouverture avec une motte de terre ou avec un léger pan de gazon; on fait entrer dans cette motte la pointe de la petite traverse, dont l'autre bout, taillé en biseau, va s'engrainer dans une des entailles que l'on a faites à un piquet qui s'enfonce dans la terre, près la taupinière de l'animal; on ne peut tendre le piége sans élever le poids de la grande trarerse ; ce mou- 
vement donuc la facilité d'arranger et de disposer les pièces attachćes aux deux ficelles, le mêne que, par son ressort ct sa pesanteur, il les tient dans une tension continuelle. La taupe se trouve arrêtée dans sa marche par la motte de terre, qui est un obstacle qu'elle ne saurait franchir sans le mettre en mouvement; la moindre secousse laisse échapper la petite traverse, dont la tête se dégage de l'entaille par la force du poids qui l'entraine; cette ficelle ne peut s'élever sans que l'autre n'en fasse autant, le lacs obéit à la même force, et la taupe se trouve prise par le milieu du corps et suspendue à la voûte de son souterrain.

\section{Piége d'Allemagne pour prendre les taupes.}

Les Allemands se servent d'une espèce de piége qui ressemble à une souricière; c'est un cylindre creux, long de deux pieds, sur lequel on tient suspendues, au moyen d'une corde qui répond au passage de la taupe, deux petites planches qui, par leur chute, traversent le cylindre, en bouchent l'orifice et emprisonnent l'animal.

\section{Piége Dauphinois.}

Les taupiers ne sont pas tous d'accord sur le point de savoir s'il y a plus d'arantage, en chassant les taupes, de laisser circuler l'air dans l'incision gue l'on fait pour tendre les piéges, ou d'y fermer tonte issue. Il parait que, dans l'un et l'autre cas, les taupes sc laisscnt prendre, et que 
l'important est de bien savoir apprécier la galcrie fréquen. tée par la taupe, pour \& tendre le piége. La plupart des taupières anciennes étaient sans ouverture à leur partie supérieure, et tels sont les piéges dont on se sert dans le Dauphiné, ce sont des cylindres creux de bois de sureau ou de hêtre, sans aucune ouverture à leur extrémité supérieure; ils sont d'une pièce et de huit à dix pouces de long, et ont une porte de tête semblable à celle des taupières ordinaires; cette espèce de piége, tout simple qu'il est, remplit parfaitement son but, et rarement la taupe s'en échappc lorsqu'elle s'y trouve renfermée. Il ne diffère de celui de M. Lafaille, $f g .15$ et 16 , qu'en ce qu'il n'y a pas de jour à l'extrémité opposée à celle où la soupape est placée.

Iroyen de prendre les taupes en Flandre et en Hollande, avec un assommoir.

Les Hollandais et les Flamands se servent, comme nous, de taupières et de piéges pour détruire les taupes, mais ils font aussi usage d'une machine qui, suspendue 'sur le passage de la taupe, tombe sur elle lorsqu'elle vient fouiller auprès, et l'assomme. Cette espèce de piége est trop connu pour en faire ici la description; nous nous bornerons à le représenter dans la fig. 19.

\section{Piéges flamands.}

Les piéges flamands ont été préconisés par Lafaille; ils 
réunissent, d'après cet auteur, des avantages que les autres n'ont pas.

Le piége de Flandre est un morceau de bois cylindrique, qui s'ouvre dans toute sa longueur, et qu'on réunit par un cercle de fer ou d'osier; la porte est cachée et ne forme aucun obstacle; elle est suspendue par une petite broche de fer assez étroite et encore plus mince, laquelle est soutenue par un fil de mème métal mis en travers du cylindre; un ressort placé dans une partie de sa longueur, et qui se courhe derrière la porte, la tient toujours fermée; ce n'est pas même sans effort qu'on oblig̨e le ressort à céder, alors la porte se lève; pour la tenir dans cette situation, on passe au-dessus du fil de fer la petite broche, dont la pointe appuie légèrement sur un de ses dentillons, tandis que la tête, que l'on a soin de courber, forme au milieu de la taupière une plaque qui sert d'obstacle à son passage. Aussitôt que la taupe touche ce point de faible résistance, la broche tourne, se dégage du dentillon, et la porte cède à l'effort du ressort qui tend à la tenir fermée, et la taupe est prise.

Taupière ou piége de Lafuille.

Bien arant Lafaille, on se servait des taupières simples et composées pour prendre les taupes; mais la plupart de celles dont il s'était servi lui-même, dans ses longues et nombreuses expériences, l'ayant convaincu que les uncs et les autres étaient plus ou moins imparfaites, il imagina de 
perfectionner celle qui était le plus en usage de son temps. Cet écrivain entre dans de trop minuticux détails sur sa nouvelle taupière, pour le suivre dans la description quil en fait.

La taupière de Lafaille est un tube de bois, $f g$. $1 \mathrm{~J}^{\mathrm{e}}$ et $16^{\mathrm{e}}$, de forme cylindrique, de nenf à dix pouces de long, et de dix-huit lignes de diamètre à l'extérieur; il parte ì l'un de ses bouts un grillage de fil de fer, ct de l'autre unc espèce de soupape, $a$, ou de porte de tôle suspendue par une charnière, $b$, et cédant au moindre effort de l'intérieur; cette soupape ne peut s'élever en dehors parce qu'elle est retenue par le bourrelet, $c$; on place le piége dans le passage du domicile de la taupe, qu'on a préalablement découvert à son extrémité ; l'air qui entre dans le passage par l'extrémité grillée du piége, porte l'animal à venir réparei le dommage, et il se précipite dans le piége qui l'attend.

Lafaille reut que l'on ne confectionne son piége qu'avec du bois un peu tendre, tel que celui de frêne et d'aubier; il cite pourtant le noyer, le sycomore et d'autres bois qui peuvent avantageusement être employés à cet usagge ; cet auteur croit aussi nécessaire de passer à travers les flammes le piége où une taupe s'est prise, afin que l'odeur cadavéreuse que cet animal laisse après lui n'éloigne pas les autres; il a cru s'apercevoir qu'une taupe qui restait longtemps morte dans le piége, tombait en putréfaction et empêchait les autres de venir s'y prendre; c'est pour cola qu'il recommande de visiter souvent les piéges, toutes les six heures au moins. Il est de fait que, pour ceux qui son? dans l'habitude de tendre des piéges aux taupes, c'est unc 
mâu aise méthode de les laisser trop longtemps, puisque, si on pose bien le pićgé, et à l'endroit où se trouve la taupe, elle ne reste jamais long-temps sans venir voir ce qu'on a fait ou défait à son ouvrage, alor's elle se prend ou elle s'échappe; dans le premier cas, l'opération est finie, et l'on peut tendre son piége ailleurs; dans le second, on cherche J'endroit où la taupe s'est réfugiée, et on avise de nouveau au moyen de la prendre.

\section{Taupiere simple.}

Les taupières sont des piéges cylindriques en bois, en fer-blanc ou en terre, d'un diamètre un peu plus large que l'est ordinairement celui de la galerie par où passe la taupe. La taupière simple, dit Rosier, est un morceau de hois de dix à douze pouces de longueur, fig. 150 et 16 ; ce morceau de bois est̂ creusé sur presque toute sa longueur, la partie qui ne l'est pas empêche la taupe de sortir ; l'autre extrémité est garnie d'une petite proéminence ou bourrelet, d'une à deux lignes de hauteur; on cloue, par la partie supéricure, une soupape en cuir, juste de la largeur du creux du bois, de manière qu'clle peut être soulevée de dehors en dedans, et non pas de dedans en dehors; cette espèce d'étui une fois préparé, on enlère, suivant la longueur, la terre qui couvre la galerie formée par la taupe, on le place dans cette galerie, et on le recouvre de terre; l'animal vient, soulève la soupape, entre, la soupape se baisse, et il est pris; mais s'il se présente contre le bout 
fermé en bois, il ouvre une autre galerie et il évite le piége. On remédie à cet inconvénient, en plaçant dans le milieu de la taupière un bouchon, soit en bois, soit en liége; alors, la garnissant d'une soupape à chacune de ses extrénités qu'on a laissée ouverte, l'animal se prend de quelque côté qu'il se présente; une fois renfermé, il ne peut plus sortir, parce que, plus il pousse la soupape, plus elle se colle contre le bourrelet, et mieux elle le ferme. Ce piége diffère peu de celui de Lafaille. Voyez les fig. 15 et 16.

L'endroit ponctué indique la séparation nécessaire lorsqu'on veut qu'il y ait une entrée à chaque extrémité.

Les taupières simples varient peu dans leur forme; ce sont presque toutes des espèces de boîtes à fourreaux, faites de plusieurs branches de sureau, qu'on fend et qu'on creuse; on rejoint ensuite ces pièces avec un petit cercle de fer.

Il n'en est pas ainsi des taupières composées, dont on se sert dans les pays septentrionaux et sur les bords du Rhin; l'usage les a beaucoup variées, et on en fait d'un grand nombre d'espèces; la plus commune de toutes est celle qui se fait avec une planche fixée à terre par quatre crochets armés de lacs, dans lesquels la taupe se prend à travers le corps. Nons allons donner la description de la taupière composée, dont on se sert dans le nord de la France et aux bords du Rhin.

\section{Taupière composée.}

Cette taupière est une des meilleures et des plus solides 
que l'on connaisse, aussi en fait-on encore un grand usage en France, surtout dans nos départemens septentrionaux. Dans un article inséré dans la Bibliothéque Physico-économique de l'année 1788, intitulé : De la manière de prenare les taupes dans les prairies et les jardins, on fit, pour la première fois, la description très détaillée de cette taupière.

La figure 15 représente la taupière tendue, et dans la situation où elle doit être dans la terre ; on y voit entrer la taupe par l'un des bouts.

$\boldsymbol{A}$ est une petite boîte sans fond, ayant la forme d'un carré long; et cinq pouces et quelques lignes de longueur sur deux pouces et demi de large; l'épaisseur de la planche peut être de trois à quatre lignes. La partie du dessus est percée de cinq trous: un au milieu, $b$, d'environ trois lignes de diamètre; un à chaque coin, $c, d, e, f$, et à quatre lignes du bois. $G$ est une gaulette flexible que l'on enterre par le gros'bout, de manière à la fixer solidement, et qu'en la ployant elle puisse faire le ressort. On attache à l'extrémité, $h$, les bouts de fil de laiton, après en avoir passé un de $c$ en $d$, et l'autre de $e$ en $f$; au même point, $h$, est aussi attachée une ficelle qui descend dans le trou, $b$; la longueur de cette ficelle ne doit être que du cinquième des fils de laiton; il y a un nœud au bout qui doit couler facilement dans le trou $b$. Quand on courbe la gaulelte, on retient cette ficelle au moyen d'une cheville, $i$, que l'on place entre le nœud et le bord du trou, $b$, mais de façon que le moindre mourement fasse tomber la eheville. La ficelle, 
ainsi fixée, empêche la gauletie, $g$, de se redresscr et làche les fils de laiton, qui font alors le demi-cercle, $k$.

Il est urgent de maintenir la boîte, soit arec des crochets piqués en terre, ou par tout autre moyen, car l'effort que fait la gaulette poar se redresser l'enlèverait.

La fig. 14 est le dessus du piéga, renversí, pour qu'on puisse le voir plus clairement.

Esage de la taupière.

Lorsqu'une taupe a fait une motte, on taupinière, dans un jardin ou dans une prairie, on déccurre cette motte pour voir la direction du passage souterrain; on cherche ensuite avec une bêche, à une distance de trois ou quatre pieds de la motte, l'ouverture par où passe la taupe. Ce passage n'est ordinairement qu'à deux ou trois pouces de profondeur. On coupe avec la bêche le terrain à côté, de la longueur et de la largeur de la taupière, c'est-à-dire de cinq pouces et quelques lignes de longueur, sur environ trois pouces de largeur; on tend la machine en la plaçant dans cette petite tranchée, en observant que ses deux extrémités, les deux bouts où se trourent les arcades, répondent exactement aux deux passages, ou plutôt aux deux extrémités du passage de la taupe. Il est entendu que les deux petites arcades sont dessous la planche et posent sur le fond de la tranchée. 
On se sert de fil d'archal ou de laiton, parce que de la ficelle ne conserverait pas la forme de cercle, et que d'ailleurs les taupes pourraient la ronger.

Quant à la cheville, elle doit toujours être placée avant que la taupière ne soit dans la tranchée, sans quoi on ne pourrait plus l'y mettre. Les laitons et la cheville étant arrangés, on place la taupière dans la tranchée, comme il a été dit; on rapproche ensuite la terre, et on laisse le moins de jour possible; comme il pourrait s'en trouver aux deux bouts de la taupière, on les bouche avec un peu de terre ou avec des gazons : il n'est pas absolument nécessaire qu'il n'y en ait point du tout. Cela fait, la machine est tendue.

Quand la taupe vient, soit d'un côté, soit de l'autre, elle entre dans une arcade, et trouve dans son chemin la petite cheville qui est au milieu, elle la pousse pour passer et la fait tomber. Le bout de la ficelle n'étant plus retenu par la cheville, s'échappe par le moyen de la gaulette qui tire continuellement cette ficelle, et laisse à cette gaulette tout le jeu de son ressort, qui la fait redresser et tirer violemment les deux fils de laiton qui forment les deux arcades, au moyen de quoi l'animal se trouve pris au travers du corps par l'une ou l'autre arcade. Au surplus, tout ceci est. beaucoup plus long à décrire qu'à exécuter.

Quand on arrive dans le jardin, on voit si la perche est détendue, ce qui annonce la prise de l'animal.

Je finirai par une observation essentielle, qui est de ne CHASSEUR TAUPIER。 
jamais placer la taupière à l'endroit même où l'animal a bouté et où il a poussé la terre en dehors, parce qu'alors il pousse la terre devant lui et en remplit la taupière, ce qui l'empêche de se prendre. Quelquefois la taupe passe à côté de la taupière, ce qui est pourtant rare, alors on déplace le piége et on le met dans un autre endroit.

Méthode des taupiers des environs de Paris.

C'est la même que celle des taupiers de la Touraine, et elle diffère peu de celle dont Lecourt fait usage. Les taupiers des environs de Paris, à l'exemple de ceux de Normandie, exercent leur talent sur les terres des fermiers de leur contrée. Ceux-ci leur donnent une certaine somme pour venir, tous les ans, chasscr les taupes qui dévastent leurs champs; ils font cet ouvrage deux fois par an, au printemps et en automne. On sent bien qu'un taupier ayant, tous les ans, une certaine circonférence de terrain à exploiter, met dans son travail beaucoup de négligence, et, pour ses intérêts, beaucoup d'indifférence; aussi, d'une année à l'autre, on nc s'aperçoit pas de la diminution des taupes sur l'héritage qu'elles ont coutume de fréquenter. J'ai resté dix ans dans le département de Seine-et-Marne, au milieu des fermiers; j'ai vu leurs taupiers, all printemps et en automne, faire la chasse aux taupes; ils en détruisaient toujours quelques-unes, mais les petits restaient, et l'année suivante il y en avait autant.

Cependant, en attaquant saramment et habilement une 
terre dévastée par les taupes, soit avec la méthode do M. Dralet, soit avec celle de Lecourt, soit même avec les moyens employés par les jardiniers normands, on doit être convaincu que les mâles, les femelles et leurs petits, ne doivent point échapper à la destruction. Est-ce donc par ignorance ou par incapacité qu'on laisse exister des taupes dans un endroit où on les attaque?

Non, répondront les taupiers de Paris. Eh ! pourquoi donc il y a-t-il toujours autant de taupes qu'il $\mathrm{y}$ en avait il y a centans, et mille fois plus de taupiers qu'on n'en voyait alors ? La réponse est facile à faire : il faut que tout le monde vive et que les espèces se conservent.

Le piége dont se servent les taupiers des environs de Paris, est une espèce de pincettes, fig. 17, constamment fermées par l'effet de l'élasticité du ressort, $a$; on tient ouvert les deux branches, $d$, au moyen d'une plaque de tôle, $b$, en la plaçant à l'extrémité, $c$. Le piége est mis dans le boyau communicateur, après en avoir enlevé la taupinière; la taupe, dans sa course, pousse la petite plaque, $b$, en essayant de passer par l'ouverture, $e$; la plaque se dérange, les pinces se ferment, et l'animal est pris.

Méthode de prendre les taupes selon Lecourt.

Lecourt fut un habile taupier, qui, dans le principe, fit cet état par délassement, et ensuite par profession; il légua, en mourant, sa science à sa nombreuse postérité, qui, 
marchant sur ses traces, est devenue, comme dit Bosc, aussi savante que lui.

Ce qu'on peut donner à Lecourt, par dessus les taupiers ordinaires, c'est un esprit observateur, un talent de juger et d'apprécier les difficultés de son art, et de les résoudre avec facilité et sans obstacle; ce qui indique que son génie l'avait naturellement porté à l'occupation à laquelle il s'est donné : cependant Lecourt n'a rien inventé pour son art; avant lui, les taupiers des environs de Paris se servaient d'une houe, d'un couteau, d'une boîte pour emporter dans leurs courses les petits objets nécessaires à leurs opérations. Lecourt n'a point inventé de piége, il a perfectionné celui dont se servaient ses confrères; il a innové quelque chose sur la forme de cet instrument. Le piége de Lecourt ressemble en tout à celui des taupiers des environs de Paris, $\hat{n g . ~} \mathbf{1 7}^{\mathrm{c}}$; seulement les avant-branches des pinces sont plus allongées; il se tend de même, et la taupe s'y prend par les mêmes moyens; toute la différence de la méthode consiste à adosser deux piéges l'un contre l'autre. La tête est en acier aplati, les branches sont en fer, leur extrémité est armée de deux crochets ployés en contre-base et en angle droit de vingt lignes. La longueur du grand piége est de sept ponces six lignes; il y en a de plus petits pour tendre dans les murs. La supériorité de ce piége fut reconnue, il y a trente ans, par la Commission d'Agriculture; elle l'approuva dans une de ses séances.

Pour opérer avec ses piéges, Lecourt fait à-peu-près comme tous les taupiers : il se met en chasse par un beau jour, et aux heures les plus farorables; est-il sur un héri- 
tage habité par les ennemis qu'il va attaruer, il explore les lieux, il juge des positions, lls'oriente, en un mot, comme un observateur habile; ensuite il cherche les taupinières qu'il appelle de passage, il les reconnaît à leur élćvation et ì leur gros volume; alors il les enlève avec sa hone, et il cherche l'endroit propice à placer ses instrumens.

Voici, d'après Cadet de Vaux, comment il opère : le passage trouvé, il l'évente, il rejette la terre, il voit les deux arceaux du passage, il les sonde, s'il le juge à propos, et leur donne la longueur du piége, à l'aide de la lame d'un couteau; il retire la terre, il la laboure légèrement, il met la détente, il pose le piége ou le place, ou plutôt les place, car on en met deux en sens opposés, l'un pour saisir la taupe quand elle gagnera son passage pour sortir du gîte, l'autre pour la saisir si elle en est absente, au moment où , revenant du travail, clle regagnera sa demeure.

On place les deux piéges en avant et tendus, on les pousse dans l'intérieur de l'arceau; on les enfonce légèrement, on en assujettit la base avec un peu de terre, on les soutient avec une petite motte inclinée, touchant à la voûte de l'arceau et posant dans le fond; enfin une poignée de terre recouvre l'appareil; la taupe a le piége en perspective, ce qu'elle rencontre est un éboulement de terre; elle fouille pour la tasser de côté, et elle se trouve prise. On place toujours le piége à l'endroit où les galeries aboutissent; douze à quinze galeries prennent naissanceidans le passagc, mais à de différentes distances.

Lecourt, pour prendre der nids de taupe, sossure d'an 


\section{$-70-$}

bord de l'endroit oủ les femelles les ont placés, ce qu'il reconnait encore à l'inspection des taupinières; Il creuse avec sa houe, il enlève le dôme qui les recouvre, et prend ainsi toute la progéniture. 


\section{DE LA DESTRUCTION}

\section{DES ANIMAUX RT DES INSECTES}

\section{NUISIBLES AU J RDINAGE.}

Il importe de surveiller avec diligence les cultures qui ont coûté beaucoup d'argent, de travail et de soins. Tant d'espérances s'attachent à leur succès, et tant d'utilité doit résulter de leur état prospère ! Il faut donc veiller efficacement à leur conservation.

Au moyen de bonnes clôtures, les animaux un peu forts n'entreront pas dans le jardin. Il ne s'agit donc que de le préserver de la voracité et des ravages des petits animaux, d'autant plus redoutables qu'ils sont difficiles à découvrir, qu'ils sont nombreux, et qu'ils se multiplient souvent par' myriades.

Araignée. Il est une variété de cet insecte vorace qui ne se borne pas à la destruction des mouches et des moucherons, ce qui serait fort utile. La variété dont nous provo= 
quons ici la perte, s'attache aux jeunes semis de carottes, et y occasionne quelquefois les plus grands ravages. Elle pique la plante lorsqu'elle vient à lever, et la fait périr sans ressourcc. On détruit, ou au moins on écarte cet insecte, en arrosant le plant à plusieurs reprises dans la journée, en commençant le matin, par une aspersion de suie écrasée très fin et délayée clans un volume d'eau assez considérable pour qu'elle ne fasse que la noircir. Il paraît que l'amer tume de la suie suffit pour offenser les araignées; j’ai d'autant plus lieu de le présumer, que j’ai obtenu le même arantage d'une infusion à froid de feuilles d'absinthe broyées dans l'cau.

Chararcors. Cetinsecte, si préjudiciable dans les champs à nos blés, ne l'est guère moins à la vigne et à quelques plantes, dont il roule et détruit les feuilles, si nécessaires à la prospérité des régétaux de tonte espèce. On écrase ces insectes lorsqu'on les décourre: ce moyen est́t toujours efficace, mais il est lent et difficle. Il faut aroir recour's à des remèdes praticables. L'odeur du chanvre et du sureau broyées dans l'eau, contribue beaucoup à les écarter; des frictions d'ail écrasé sur les tiges des plantes attaquées, achèvent de mettre en fuite ces insectes, qui finissent par désemparer un terrain où ces odeurs les importunent.

Chenthes. Le meilleur moyen de se présciter de ces 
hideux animaux est de recourir à l'échenillage : il faut, avant qu'elles éclosent, enlever tous les anneaux ou bourrelets de petis œufs fort durs qui entourent les branches des arbres, et les bourses qui renferment d'autrcs œufs ou cocons. On doit s'empresser de les jeter au feu. Quelque soin (qu'on apporte à cette facile destruction, il peut écliapper quelques petits dépôts, quelquefois placés dans de vieilles écorces. Il peut aussi venir des chenilles du voisinage où l'on n'aurait pas mis le même soin ả leur poursuite; alors avant le lever du soleil on enlève ces insectes réunis et tapis sur quelques feuillages où le froid de la nuit les force de se retirer. On peut aussi placer un réchaud garni de charbon allumé sous les plantes attaquées : un peu de fleur de soufre jeté sur ce charbon vient à s'enflammer, et, par son odeur, asphysie à l'instant même les chenilles, qui tombent sans force. Ce moyen exige beaucoup de précaution, car il faut soigneusement éviter de brûler ou même de chauffer tropu fortement les ramcaux et les feuillages qui, à cette époque;: sont fort tendres. On fait aussi périr les chenilles par des aspersions, soit d'eau dans laquelle on a fait fondre du savon noir, soit de jus de fumier. L'odeur du tabac les écarte aussi, mais ce moyen est peu praticable.

Courtilliere ou Taupe-grillon. Cet insecte se retire sous terre, où il déplace les racincs et bouleverse parfois le terrain descultures nouvelles. On emploie l'eau de savon noir, ou l'eau mélangée arec un peu d'huile commune, gule l'on verse avec l'arrosoir à bec dans le trou où se re- 
tire l'insecte. Cette retraite se reconnait à la forme arrondie de terre remuée qu'il a poussée dehors, à la manic̀re de la taupe. La courtillière, comme beaucoup d'insectes, périt dès qu'elle a été atteinte par l'buile ou tout autre corps gras.

Fouryrs. Lorsqu'on s'est assuré du líeu où elles se retirent, il faut les cerner et jeter assez d'eau bouillante pour anéantir la fourmilière. Si par hasard elle se trouvait trop près d'une plante importante, on attirerait les fourmis à quelque distance de là, en bouleversant leur travail; en les inquiétant pour les forcer à s'enfuir vers le point où il est facile de les attirer, en enduisant d'un peu de miel un pot que l'on renverse, et sous lequel on ménage un passage. Aussitôt qu'elles s'y sont établies, on l'emplit d'eau bouillante. Quand on peut craindre que cette eau n'atteigne des plantes ou des racines utiles, il suffit de jeter sur la fourmilière un peu de chaux vive, sur laquelle on verse de l'eau froide. Ce moyen est très efficace et vaut mieux que les arrosemens d'eau de chanvre dont nous arons parlé plus haut, et qui seraient ici à-peu-près inutiles, puisqu'ils ne feraient que déplacer le mal. L'huile dont on arroserait les fourmis, les ferait aussi périr. La grosse fourmi, dont on se débarrasse ensuite, introduite dans le jardin, y détruit promptement la petite espèce. On empêche encore ces insectes d'attaquer les vases de fleurs, en plaçant dessous un plateau de terre cuite rempli d'eau qu'elles n'osent tra- 
verser. Un anneau de laine et de crin roulé autour du trone d'un arbre; une bande de cuir circulaire enduite de glu ou d'une peinture à l'huile entretenue humide, ne leur permettent pas de parvenir aux branches et d'en dévaster les feuillages et les fruits.

GuÊpes et Frelons. On en prend beaucoup au moyen, seit de petits filets dont on se sert pour attraper les papillons, soit de carafes remplies au tiers avec de l'eau miellée; mais le plus sûr moyen est de chercher leur retraite, qui est ordinairement dans la terre, dans un trou de mur ou de quelque vieil arbre. Dès que cette retraite est découverte, on vient la nuit, le plus tard que l'on peut, avec une pelotte d'argile ou de mastic de vitrier, et l'on bouche exactement l'ouverture du guêpier. Il suffit ensuite d'y répandre, au moyen d'un petit entonnoir, une quantité d'eau bouillante suffisante pour détruire tous ces insectes.

Hannetons. Ce n'est pas comme hanneton que cet insecte est redoutable aux jardins, où l'on peut facilement l'écraser après avoir secoué les rameaux des arbres qu'il attaque; c'est lorsqu'il n'est encore que man ou turc, et vivant sous terre, qu'il fait le plus de ravages, en dévorant les racines des jeunes plantes, et même des arbres les plus forts. Il faut le chercher avec soin en hêchant, pour le tuer, et lorsqu'on s'aperçoit qu'une jeune plante, pourtant vigou- 
reuse, vient tout-à-coup à se flétrir, on doit en découvrir les racines, où il est facile de trouver l'insecte; on l'écrase avec facilité, parce qu'il n'est pas du tout agile. C'est principalement aux racines charnues de la laitue et du fraisier qu'il s'attache de préférence, et c'est par-là qu'il faut toujours commencer ses recherches.

Limaces, Limaçots, Escargots. Il est bien difficile de parvenir à la destruction de ces insectes, dont on peut pourtant prévenir une partie des ravages, en les poursuivant, ver's la fin de février, dans les monceaux de cailloux et les gerçures de murailles, où ils trouvent pour l'hiver un abri dans lequel ils s'engourdissent. Il est facile de les y tuer. Pendant la belle saison, qui est l'époque de leur dévastation, on doit, dès le matin et vers le soir, et surtout lorsqu'il a plu, les chercher sur le sol et les jeunes plantes. On ic enlève dans des pots, pour les livrer aux volailles ou pour les écraser. Quand il ne s'agit que de préserver un petit nombre de plantes ou un jeune semis, on jette çà et là de la suie en poudre, dont l'excessive amertume chasse tons les insectes.

Muzors, Rats, Sounis. De l'arsénic pulvérisé et mêlé avec de la farine, est un bon moyen pour empoisonner ces animaux; mais il en peut résulter des accidens, quoique 
pourtant les chats ne mangent guère la farine, non plus que les autres animaux domestiques, parce que, sous la forme pulvérulente, elle a peu d'attrait pour cux. C'est aux ratic̀res, aux quatre-de-chiffres, qu'il faut avoir recour's, et ne pas craindre de les multiplier après les avoir convenablement amorcés. Le poison dont nous avons parlé peut s'employer sans aucun inconvénient, en ayant soin de le placer au fond des trous d'une petite pièce de bois percée par une forte tarière. Le mulot seul peut s'y introduire, et il n'est pas à craindre que des animaux utiles y puissent atteindre. Le rat est plus difficile à prendre : c'est de la ratière et du quatre-de-chiffre qu'il faut se servir contre lui. Le piége à bascule, placé sur un petit cuvier à demi-rempli d'eau, est encore un très bon moyen pour détruire beaucoup de rats et de mulots. Pour cet effet, on établit sur le cuvier une petite planchette formant bascule. Au moyen d'un fil de fer recourbé, fixé à l'un des hords du cuvier, on suspend un appât quelconque, vers lequel l'animal ne manque pas de se rendre. Aussitôt qu'il en approche, la bascule joue, l'animal tombe dans l'eau, ct la bascule se rétablit pour former le même piége à tous ceux qui viendront à se présenter.

Pucerons. Quelques plantes sont parfois infectées par une grande quantité de ces petits insectes, que leur couleur empêche d'apercevoir de loin, et que leur nombre rend très fàcheux aux feuilles et aux jeunes tiges qu'ils atta- 
quent. Des injections d'eau de suie et d'eau de savon noir, des fumigations faites avec du tabac ou de la fleur de soufre, sont les remèdes les plus efficaces pour détruire les pucerons.

Trquer, Vers de terré, etc. C'est avec une infusion de substances amères, telles que la suic, le brou de noix, les feuilles du noyer, celles de l'absinthe, celles de la rue, que l'on doit arroser la terre où l'on voit ces insectes nuire aux plantes. Ces arrosemens les chassent et en font même périr un grand nombre.

Nous ne parlerons pas des oiseaux qui font quelquefois une guerre si dévastatrice aux fruits et aux légumes. Le geai, le merle, les moineaux, les mésanges, et une foule d'autres oiseaux, attaquent les cerises, les guignes, les petits-pois, les graines qui commencent à mûrir des salsifis et de plusieurs autres légumes. Quelquefois on se contente de les épouvanter avec de vieux vêtemens de couleur prononcée, avec des animaux empaillés, avec des moulinets bruyans tournant au moindre vent; on en prend aussi avec la glu; on les effraie à conps de fusil. Tous ces moyens sont fort peu efficaces malheureusement. Il faut les employer tous à-la-fois et ne pas se rebuter. Le livre intitulé : les Secrets de la Chasse aux Oiseaux (1) forme un gros

(1) Secrets de la Chasse aux Oiseaux, contenant la manière de fabriquer les Filets, les divers Piéges, Appeaux, ete.; l'Histoire naturelle des Oiseaur qui ge trouventen France; l'art de los élever, de les soigner, de les 


\section{$-79-$}

volume, et c'est le cas d'y chercher les recettes que cet utile ourrage contient.

guérir, et la meilleure méthode de les empailler. Ouvrage orné de huit planches, renfermant plus de quatre-vingts figures; par $\mathbf{M}$. $\mathbf{G}^{* * *}$, amateur. Un volume in-1 2. Prix : 2 fr. 50 cent., et 3 fr. 25 cent. par la poste.

Cet ouvrage de $M . \mathrm{G}^{\star \star \star}$, qui prend le titre modeste d'amateur, est un Traité complet de la chasse aux oise: ux; non-sculement on $y$ apprend tous les secrets de cette chasse, mais on y trouve encore une nomenclature des moyens de conservation que l'ornithologie a imaginés. Veut-on perpétuer le souvenir d'un oiscau chéri que la mort cruelle a ravi à sa maîtresse éplorée? on a recours a chapitre qui traite de l'art d'empailler les oiseaux. S'agit-i de se procurer vivant un des mille habitants des plaines de l'air? il ne faut qu'étudier le chapitre des lacets, des filets, des nappes, etc. Grâce au livre de M. $G^{\star \star *}$, on peut aussi apprendre à guérir la gent ailée des sans nombre qui l'assiègent. 


I'. II.

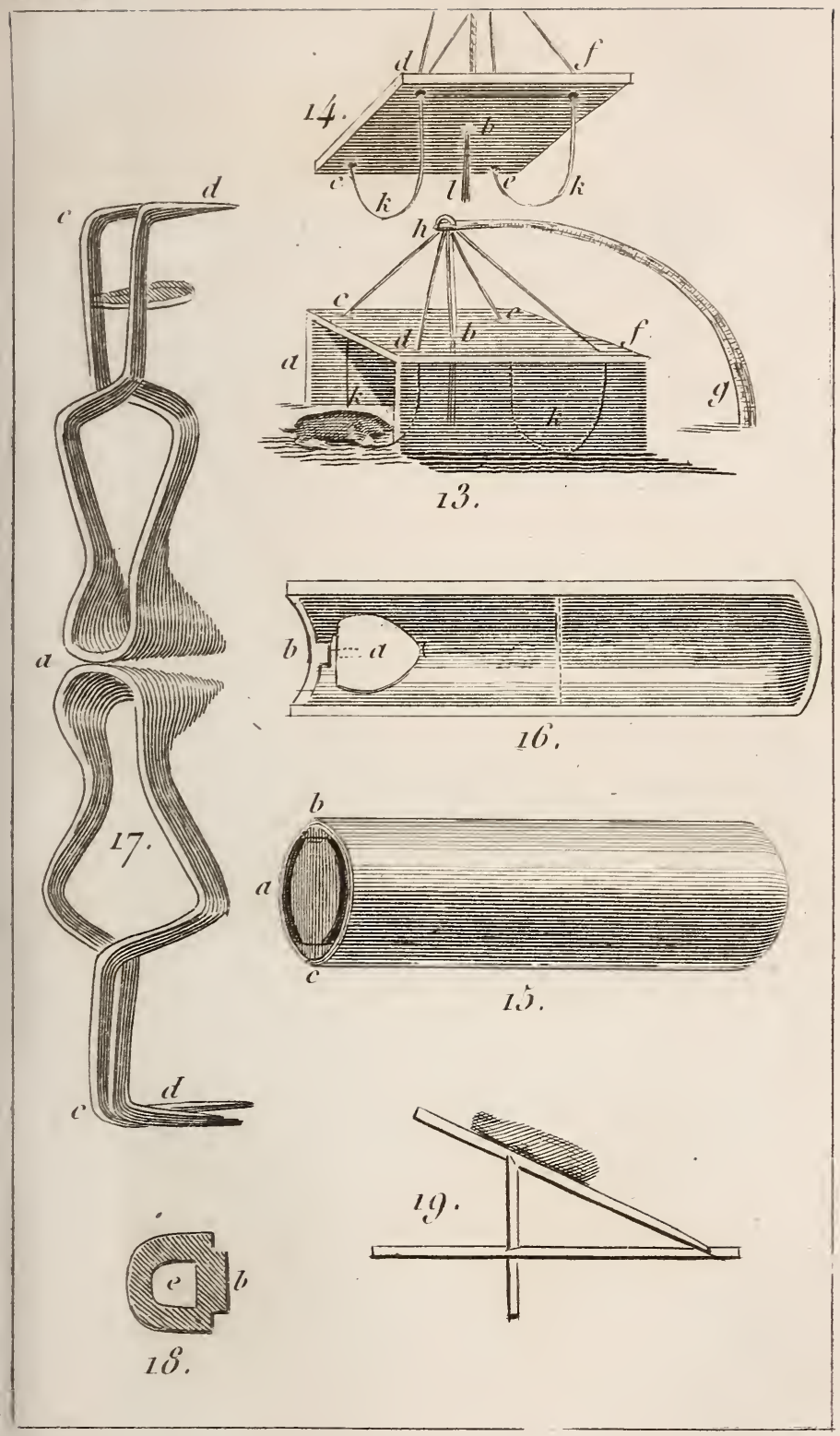

Chasseur taupier. 



\section{TABLE DES NATIERES.}

Pages,

odtuction. . . . . . . . . . . 5

coire naturelle de la Taupe. • . . . . 11

connaissances pour faire arantageusement la hasse aux Taupes. . . . . . . . . 24

divers Moyens de faire la chasse aux Taupes. $\quad 35$

mojens de faire fuir les Taupes du lieu où lles se sont établies. • . . . . . . I I .

substances végétales et minérales que l'on emloie pour faire périr les Taupes, et de l'opinion ue l'on doit avoir des appâts empoisonnés. . hode naturelle de prendre les Taupes. . . 40 rode de prendre les Taupes avec des chiens. armes à feu comme mogen de détruire les ıupes. 
Nanière de prendre les Tals pis arec de petits faisceaux de bois épineux. • . . . . . . 46

Manière de prendre les Taupes arec un pot de terre. Mianière de prendre les Taupes arec des hameçons. 48

Moyen de prendre les Taupes dans quelques con-

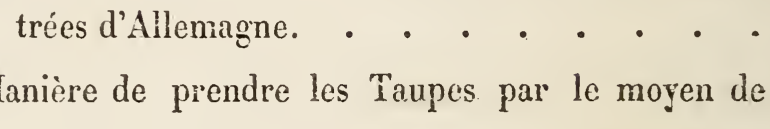
l'eau.

Méthode de prendre les Taupes, par d'Aurignac, publiée par M. Dralet. • • • . • . I Id.

Moyen analogue à celui de d'Aurignac. . . . 54

Piége des Anciens. • . . . . . . . 56

Piége d'Allemagne. • . • . . . . . 57

Piége Dauphinois. • • . . . • . . . I Id.

Assommoir dont on se sert en Flandre et en Hollande.

Piéges Flamands.

58

Taupière ou Piége de Lafaille. . . . . . 59

Taupière simple. • . . . . . . . . 61

Taupière compasce. . . . . . . . . 62

Usage de la taupière. . . . . . . . . 64

Méthode des Taupiers des cnvirons de Paris. . . $\quad 66$

Méthode de Lccent. . . . . . . . . 67

Araignéc, . . . . . . . . . 71

Charancons. . . . . . . . . T 
Chenilles. . . . . . . . . . 72

Courtillière. . . . . . . . . . . 75

Taupe-Grillon. . . . . . . . . $I d$.

Fourmis. . . . . . . . . . . 74

Frelons. . . . . . . . . . . 73

Guèpres. . . . . . . . . . . . $\mathbf{I} d$.

Hannetons. . . . . . . . . $\quad l d$.

Escargots. . . . . . . . . . 76

Limaces. . . . . . . . . Id.

Limaçons. . . . . . . . . . Id .

Hulots. . . . . . . . . ld.

Rats. . . . . . . . . . . Id.

Souris. . . . . .. . . . yd.

Pucerons. . . . . . . . . . . $\pi$

Tiquets. . . . . . . . . . . . 78

Vers de terre. . . . . . . $I d$.

FIN. 


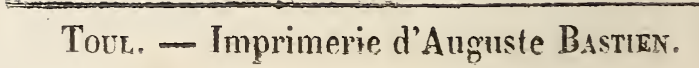




\section{- JANVIER 1859. -}

IN. B. Comme il existe क Paris deux libraires du nom do RORET, l'on sst prié de bien indiquer l'adresse.

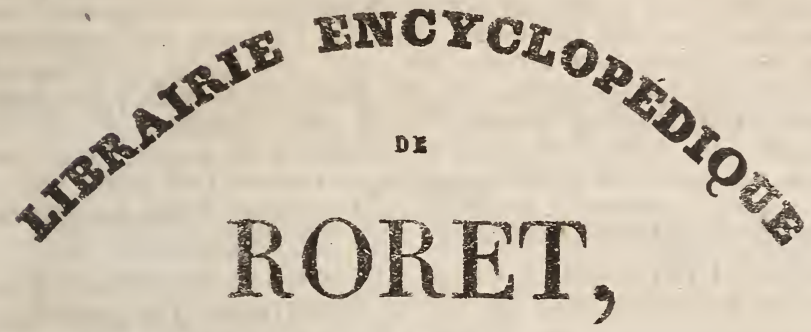

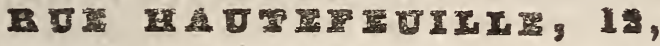

AE COIN DB LA RUR SERPERTE;

\section{A PARIS.}

Celte Librairie, entiẻrement consacrée aux Sciences et à l'Industrie, fournira aux amateurs tous les ouvrages anciens et modernes en ce genre, publiés en France, et fera venir de l'Etranger tous ceur que l'on pourrait désires.

\section{DIVISION DU CATALOGUE}

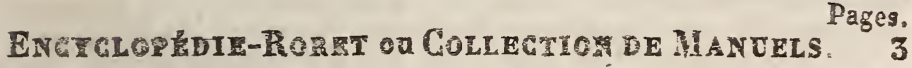
SUITRE A RUFror, format in-8 $8^{\circ} \cdot$. . . . 28 SUITgg 4 RUPFON, format in-18. . . . . . 32

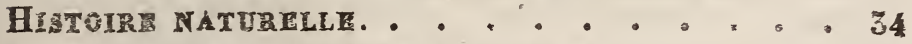

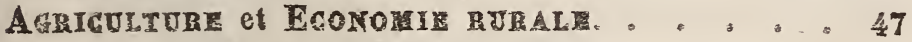

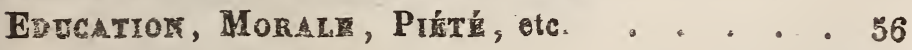
OTVRAGES DIVRRE. . . . . . . , . . . 67

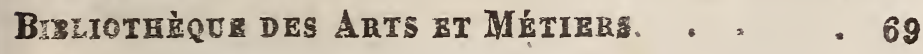




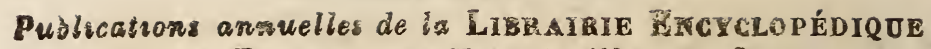
DH RORET, rue Hautefenille, sy 12.

LE TECHNOLOGISTE, ou Archives des Progrès de l'Industrie FRANÇAISE ET ÉTRANGERE, publié par une Société de savants et de praticiens, sous la direction de M. MALEPEYrE. Ouvrage utile ans manufacturiers, aux fabricants, aux chefs d'ateliers, aux ingénieurs, aux mécaniciens, aux artistes, etc., etc., et à toutes les personnes qui s'occupent d'arts industriels $20 \mathrm{e}$ année. Prix : $18 \mathrm{fr}$. par an pour Paris; 21 fr. pour la province, et 24 fr. pour l'Etranger.

Chaque mois il paraît un cahier de 48 pages in -8 , grand format, renfermant des figures en grande quantité, gravées sur bois et sur acier.

Ce recueil a commencé à paraître le 1 er octobre 1859. Le prix des 20 années est de $18 \mathrm{fr}$. chăacune.

L'AGRICULTEUR-PRA TICIEN, REVUE D'A GRICULTURE, DE JARDINAGE ET D'ECONOMIE RURALE ET DOMESTIQUE. Voy, page 47.

ALMAN.ACII ENCYCLOPÉDIQUE RÉCRÉATIF ET POPULAIRE pour 1859 , d'après les travaux de savants et de praticiens célèbres. 1 vol. in-16, grand raisin, orné de jolies gravures. $\quad 50 \mathrm{c}$. Il a paru 20 années de eet Almanach, à $50 \mathrm{c}$. chaque.

BELGIRUE HORTICOLE (LA), par CH. HORREN. Journal paraissant chaque mois depais juillet $18 \% 0$, et renfermant $24 \mathrm{pl}$. col. par an. (70 année.) Prix :

16 fr. 50

BULLETIN DE LA SOCIÉTÉ INDUSTRIELLE DE MULHOUSE. Il a paru 5 séries de ce recueil. Les deux premières, format in-8, et la troisième, format in $\mathbf{8}$ grand papier.

La première a commencé en $\mathbf{1 8 a} 6$ et finit en 1810. Elie comprend les cahiers 1 à 65 , ou vol. 1 à 15 ; pris: $9 \mathrm{fr}$. le vol.

La seconde a commencé en 1840 et finit ell $185 \% 4$. Elle comprend les cehiers 66 à 125 , ou vol. 14 à 25 ; prix : 12 fr. le vol.

La troisième, format in-8 grand papier, a commencé en 1854 et ge continue. L.e prix de la souscription pour Paris est de 15 fr. par vol, composé de 6 cahiers, et du 18 fr. pour la provirice. Les cahiers 126 à 14 sont parus. Chaque numéro des trois séries se vend sćparément 5 fr. BULLETIN DELA SOCIÉTÉ FRA NÇAISE DE PHOTOGRAPHIE.

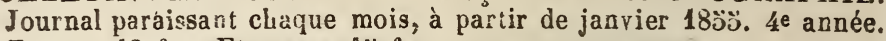
France, 12 fr.; Etranger, 10 fr.

LE GARDE-MHE UBLE, JOURAL D'AMUELEMENT; 5 a plancha

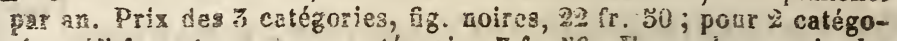

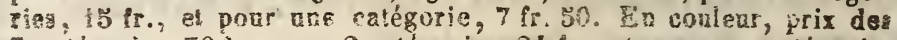
3 cstégories, 36 ir.; pour 2 cztégorien, 21 tr., et pon: une cátégorie.

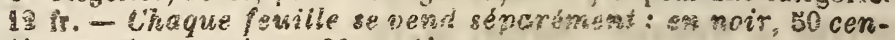
times el en couleur, 80 centimes. 


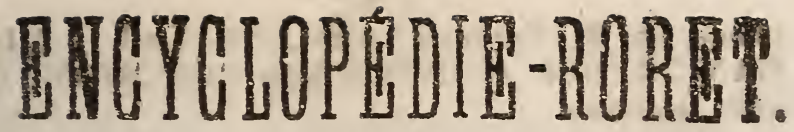

COLLEG'TION

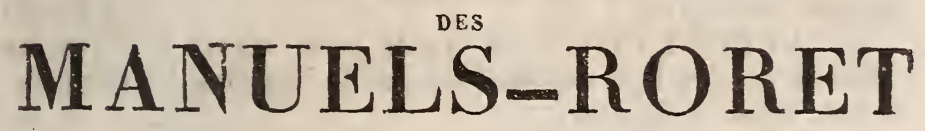

FORMANT

ONE GICYCLOPÉDER DES SCCEICES ET DRS ARMS,

FORMAT IN-18;

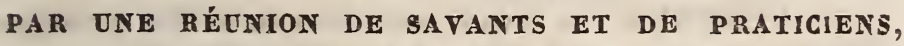
Messieurs

Ahoros, Arsenne, Barthelemy, Bitaille, Benuyalet, de Bayay, Biot, Biret, Biston, Boisdural, Boitard, Bosc, Boutereau, Boyard, Boyer de Fonscolonbe, Cahen, Capron, Chaussier Chevrier, Choson, Constantin, D'Orbigny, DE Gayffier, De lafage, de lépinois, De Montigny, De Pareto, De Siebold, De Saini-Victor, De Valicourt, Paulin Désormeaux, Jules Desportes, Drapier, Dubois, Dujardin, DupuisDelcourt, Francozur, Gallas, Garnier, Gentilhomie, GiQUel, Guilloud, Hamel, Hervé, Huot, JaNvier, Julia-FonTENELle, JULIEN, KNECHT, LACORdAipe, LACroix, LAGARDE, Landrin, Launay, Led'Huy, Séhastien Lenormand, Lesson, LORIOL, MAGNIER, MALEPEYRE, MARGEL DE SERRES, MATTER, Miné, Muller, Nicard, Noel. IImo Pariset, Paulin, Jule Pautet, Pedroni, Ponsin, Rang, Rendu, Richard, Riffault, Roussel, Schmit, Scribe, Spring, Stannius, Tarbé, Terquer,

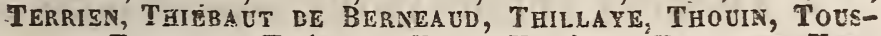
SAINT, TOUSTAIN, TRÉMERY, TRUY, VALÉRIO, YASSEROT, VLUQUELIN, VRBOIER, VERGNAUD, WALKER, WITH, YVART, etce, atc.

Les personnes qui auraient quelque chose à faire parvonir dans l'intérêt des sciences et des arts, sont priées de l'envoyer franc de port à l'adresse do M. lo Directeur do $l$ '璒ncyclopédie-Roreit, ruo Hantefeuille, n. 12, à Paris.

Tous les Traités se rendent séparément. Les ourrages indiqués sous presse paraîtront successivement. Pour receroil chaque volume franc de port, l'on ajoutera 50 c. La plupart des rolumes sont de 3 à 400 pages, renfermant des plece ches parfaitement dessinées et gravées.

MAINUEL POUR GOUVERNER LES ABEILLES en retirer un grand profit, par M. RADOUAN. 2 vol. 6 fr. ACCORDEUR DE PIANOS, mis à la portée de tout 
le monde, par M. Grorgio A RMElliNo. 1 val. 1 fr. 25

MANUEE DES AGIDES GRAS GONCRETS, voyez sougies stb́ariques.

- ACTES SOUE SIGNETURES P而YVES an ma-

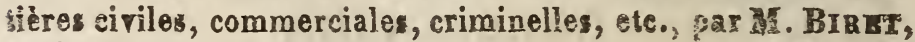
ancien magistrat. 1 rol.

2 fr. 30

- AEROSTATION ou Guide pour servir à l'histoire sinsi qu'à la pratique des Ballons, par M. DUP UIS-DELCOURT. 1 rol. orné de figures.

$3 \mathrm{fr}$.

- AGENTS-7OYERS, voyez Constructeur en général.

- AGRICULTURE ELEMENTAIRE, à l'usago des scoles primaires et des écoles d'agricalturo, par Y. RENDO. ('utorisé par l'Université.)

1 fr. 25

- ALGEBRE, ou Exposition élémentaire des principe ie cette science, par M. TERQOEL. (Ouvrage approuvé par is' iniversite.) 1 gros vol.

$3 \mathrm{fr} .50$

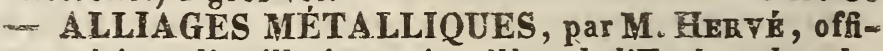
cier supérieur d'artillerio, sncien élève de l'Ecole polytechnique. 1 rol.

$3 \mathrm{fr} .50$

Ouvrageapprouve pa le Comité d'artillerie, qui en a fait prendre un nombre pour les écoles, les forges el les fonderies.

- ALLUMETTES CZIMIQUES, COTON et PAPIER POCDRE, POUDRES et AMORCES FULMINANTES; dangers, accidents et maladies qu'elles produisent; par le docteur Roossel. 1 vol. orné de figures. 1 fr. 50

- AMIDONNIER et VERMICELEIEB; psz M. It decteur Monir. 1 vol. arec figures.

3 fis.

- AMORCES FULMINANTES, voyez Aliumette chimiques.

- ANATOMIE COMPAREE, par MM. de SIEBOLd et GTannios; traduit de l'allemánd par MM. Sprifg et LAzORDAIR , professeurs à l'Université de Liége. 3 vol. ensemble de plus de 1200 pages, prix 10 fr. 50

- ANECDOTIQUE, ou Choix d'Anecdotes anciennes et zodernes, par madame CeLNART. 4 rol.

$7 \mathrm{fr}$.

- ANIMAUX NUISIBLES (Destructeur des) à l'agriculture, au jardinage, etc.'; par M. Yérandr. 1 vol. orn de planches.

$3 \mathrm{fr}$.

- 20 Partie, contenant les HYLOPHTHIRES ER LEURS ENNEMIS, ou Description et Iconographio des Insectes les plus nuisibles aux forêts, arec une méthode pouz apprendre à les détruire et à ménager ceux qui leur font la zwerre, à l'usage des forestiers, des jardiniers, etc.; par 
MM. Batzebure, Da Corberon ol Boisdeval. 1 rol. erné de 8 planches : pris

2 fE. 30

MANUEL DE CA TAILLE DES ARBRES FRUITIERS, contenant les notions indispensables de Physiologi régétale; un Précis raisonné de la multiplication, de la plantation et de la culture; les vrais principes de la taille ot leur application aux formes diverses que reçoivent les arbre fruitiers, par M. L. BE BAVAX. 1 vol. orné de figures. 3 fr.

- ARGHEOLOGIE, par 解. NICARD. 3 volumes aves

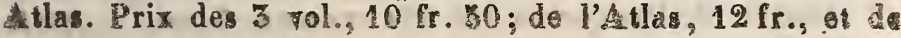
l'ourrage complet:

22 fr. 30

- ARCHITECTE DES JARDINS, on l'Art de les somposer et de les décorer, par M. Borrabi. rol. aros Atlas do 140 planches.

$15 \mathrm{fr}$.

- ARCHITECTE DES PONUMENTS RELI GIEUX, ou Traité d'Archéologio pratique, applicable la Testauration et à la construction des Eglises, par M. ScH gros volume arec Allas contenant 20 planches.

$7 \mathrm{fr}$.

- ARCHITECTURE, ou Traité de l'Art de bâtir, pą M. Tozssaint, architecte. 2 vol. ornés do planchen. 7 ff. -ARITHMÉTIQUE DUMONTRE E, par MM COLLIN ot TREMERY . 1 vol.

2 fr. 30

-ARITHMÉTIQUE COMPLEMENTAIRE, ou Recueil do Problèmes nouveaux, par M. Togeges. 1 vol. $1 \mathrm{fr} .79$ ARMURIER, Fourbisseur et Arquebusier, par M. Paulin DÉSormeadx. 2 rol. avec figures.

$6 \mathrm{fr}$.

- ARPENTAGE, ou Instruction élènentaire sur cet art at zur celui de lever les plans, par M. LAcrorx, de l'Institut. MM. HogARD, géomètre, el VASSEROT, apocat. vol. avec Ggures. (Autorisé par I'Université.) $2 \mathrm{fr} .50$

- ART MILIT ARE, par M. VRRGIADd a vol. aree figures. $3 \mathrm{fr}$.

- ARTIFICIER, Poudrier et Salpêtrier, par M. VBRGKAUD, colonel d'artillerie. 1 vol. orné de planches. $3 \mathrm{fr}, 50$ - ASPIRANTS aux fonctions de Nolaires, Grefiers, Avocats à la Cour de Cassation, Avoués, Huissiers et Commis. saires-Priseurs, par M. Combes. 1 rol.

3 fr. 50

- ASSOLEMENTS, JACHERE ot SUCCESSION DES SULTURES, par M. Victor YYABT, de l'Institut, avec des notes par M. Victor RENDE, inspecteur de l'agricalture. 3 rol.

10 fr. 30

- ASTRONOMIE, ou Traité élémentairo de cetto 
science, de W. Herscheri, par M. VerGmaUd. 1 vol. orné de planches,

3 fr. 30

MANUEL D'ASTRONOMIE AMUSANTE, traduit de l'anglais, par A.D. VergNa Ud. 1 vol. arec figures. \& fr. 50

- BALlONS, voyez Aérostation.

- BANQUIER, Agent de chango et Courtier, par MM. Peuchet ot Tremery. 1 vol.

2 fr. 50

MANUEL OU BARÊME COMPLET DES POIDS EW MESURES, par M. BAGILET. 1 rol.

$3 \mathrm{fr}$.

- BIBLIOGRAPHIE UNIVERSELLE, par MM. F Damis, P. Pinçon at De Martonne. 3 rol. $20 \mathrm{fr}$.

Le même ouvrage, grand in-8 à 5 colonnes, papier collé pour recevoir des noles.

$25 \mathrm{fr}$.

- BIBLIOTHÉCONOMIE, Arrangement, Conservation ê Administration des biblinthèques, par L.-A. CoxSTAXTIR. 1 vol. orné de figures.

3 fr.

- BIJOUTIER, Jnaillier, Orfèvre, Graveur sur métaux et Changeur, par M. JULIA DR FonTEKELLE. 2 rol. $7 \mathrm{fr}$. - BIOGRAPHIE, ou Dictionnaire historique abrégé de grands hommes, par M. NoEL, inspecteur-général des étuder. \& vol.

$6 \mathrm{fP}$

- BLANCHIMENT ET BLANCHISSAGE, Nettoyago et Dégraissage des fil, lin, coton, laine, soie, etc. : par 11. IVLIA et RoUGET DE L'IsLE. 2 vol, ornés de pl. 6 fr.

- BLASON, ou Traité de cet art sous le rapport archéologique et héraldique, par M. Jules PADTET, bibliothécairo de la ville de Beaune. 1 vol. orné de planches. 3 fr. 50

- BLEUS ET CARMINS D'INDIGO (Fabricant de), par M. Félicien CaproN, de Dôle, 1 volume. $1 \mathrm{fr} .50$

- BOIS (Marchands de) et de Charbons, ou Traité de ce commerce en général, par M. MARI DI LISLs. 1 voumae arec figures.

3 fr.

- BOIS (Manuel-Tarif métrique pour la conversion et la éduction des), d'après le système métriquo, par M. LosBARD. 1 rol.

2 fr. 30

- BONNETIER ET FABRICANT DE BAS, pas MM. Leslanc el Preaud-Galtor. 1 rol. arec fig. 3 fr.

- BOTANIQUE, Partie élémentaire, par M. BoITard. 1 rol. arec planches.

5 fr. 50

ATLAS DE BOTANIQUE pour la partie élémentaire, renfermant 36 planches. Prix

$6 \mathrm{fr}$.

- BOTANIQUE, partie, FloRE FRANGAISk, ou Description synop tique des plantes qui croissent naturellement sur 
lo sol français, par M. le ă BoISdUvar. 3 gr. v. 10 fr. 50 AtLas DE zotarigur, composé de 120 planches, reprósantant la plupart des plantes décrites dans l'ouprage cim dessus. Prix : Fig. noires. 18 fr.; figares coloriées. 36 fr.

MANUEL DU BOTTIER ET CORDONNIER, PaT M. MoRIr. I vot. avec figures. 3 fr.

- BOUCHERIE TAXEE, ou Code des Vendeurs et des Acheteurs de Viande, par UN MAgistrat. 1 vol. 1 fr. 50 Tablead re la Boucherie Taxée, in-plano. 75 c. - BOUGIES STEARIQUES, et fabrication des acides gras concrets, otc., etc., par M. MalepeyRe, un vol. orne de planches.

$3 \mathrm{fr}$.

BOULANGER, Négociant en yrains, Meunier st Constructeur de Mouling, par MM. BREOrT et JULIA DR Fontrenelle. 2 vol. arec figures. 7 ir.

- BOURRELIER ET SELLIER, par M. ERBEUR. 1) Tolume orné de figures.

s 1 ir.

- BOURSE ET SES SPÉCULATIONS mises à la porsée de tout le monde, par M. le Président Borard. 1 vol. do s28 pages.

2 fr. 50.

- BOUVIER ET ZOOPHILE, ou l'Arí d'élever et de soi-

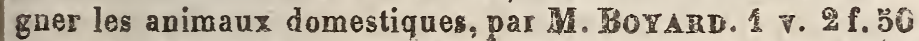

- BRASSEUR, ov l'Art do zaire toutes sorte a Bières, par M. VerGNAUD. 1 rol. 3 fro

- BRODEUR, ou Traité complètdo cet Arí, par madamo Coglinart. 1 vol. avec un Atlas de 40 pl. of fro - CADRES (fabricant de), Passe-Partout, Châssis, Encadrement, etc., par M. BE SAINT-VIGTOR, volume orné de figures.

1 fr. 30

- CALENDRIER (Théorie du) et Collection de tous les calendriers des années passées et futures, par M. TRA cosor, professeur à la Faculté des sciences. 1 vol. $5 \mathrm{fr}$.

- CALLIGRAPHIE, ou l'A ri d'écrire en peu de leçon:, par M. Tremery. 1 vol. ayec Atlas. 3 fro CANOTIER, ou Traité uxirersel et raisonaé de set Art, par UN LOUP D'EAU DOVGE; joli vol. orné de 50 vigneites sur bois.

$1 \mathrm{fr} .73$

- CARTES GÉOGRAPHIQUES (Construction et Des. siza des), par M. Perrot. 1 vol. orné de p1. 2 fr. 50

- CAOUTCHOUC, GUTTA-PERCHA, GOMME FACTICE, Tissus imperméables, Toiles cirées et Cuirs vernis, par M. Padlin-Desormeaux. 1 vol. orné de fig, 3 fr. 50

- CARTONNIER, Cartier et Fabricant de Cartonnage, par M. LEßRUN. 1 vol, orné de figures.

3 fr. 
MANUEL DU GHAMOISEUR, Pelletier-Fonrreur, Maroquinier, Mégissier et Parcheminier, par M. JoliA DE Fontenelle. 1 vol. orné de planches.

$3 \mathrm{fs}$.

- CAANDELIER, Cirier et Fabricant de Cire à cacheter, par M. Lenormand. 1 gros v. orné de pl. $3 \mathrm{fr} .50$

- CHAPEAUX (Fabricant de), par MM. Cuoz, F. et J TLIA DE FONTENELLE. 1 vol. orné de planches. 3 fY.

- CHARCUTIER, ou l'Art de préparer et de conserver los différentes partios du cochon, par M. Bareve. I rol. arec figures.

2 fr. 5

- CKARPENTIER, ou Traité simplifié de cei Art, par MM. Hañs et Biston. 1 vol. orné de $14 \mathrm{pl}$. $3 \mathrm{fr} .50$

- GEARRON ET CARROSSIER, ou l'Art de fabriguer loutes sortes de Voitures, par MM. LEBRUR, LFBoy 3t MALEPEYRE, 2.rol. ornés de 14 planches. $6 \mathrm{fr}$.

- CHASSELAS, sa culture à F ontainebleau, par un vigneron des environs. 1 vol. avec figures. $1 \mathrm{fr} .7 \mathrm{c}$

- CHASSEUR, contenant un Traité sur toute espèce de thasse, par MM. BoYARD et DE MEŔSAR. 1 rol. avec figures et musique.

$3 \mathrm{fr}$.

- CHASSEUR-TAUPIER ou l'Art de prendre le Taupes par des mojens sûr et faciles, par M. RÉ DARÈS, 1 volume orné de figures.

90 cent.

- CHAUDRONNIER, Description complète et détaillé so toutes les opérations de cet Art, tant pour la fabrication les appareils en cuivre que pour ceux en fer, etc.; par MM. JuLliex et VAlenro. 1 pol. arec 16 planches: 3 fr. 50

- CHAUFOURNIER, contenant l'Art de calciner la Eierro à chaux et à plâtre, de composer les Mortiers, les Ci.. sents, etc., par MM.BISTON et MAGNIER. 1 v. avec fig. 3 fr.

- CHEMINS DE FER (Construction de), contenant des Etudes comparatives sur les divers systèmes de la voie et du matériel, le Formulaire des charges et conditions pour l'établissement des Iravaux, etc., par M. E. With. 2 v. arec atlas. 7 f. - CHEVAL (Education et hygiène), par M. le vicomte de Montigny, 1 vol. crné de 6 planches.

CHIMIE AGRICOLE, par MM. DAFY ef VER GNAED. 1 vol. orné de figures. 3 fr. 30

- CAIMIE AMUSANTE, ou Nourelle Récréation chimiques, par M. VRRGNADD. 1 vol, orné de figuree. $3 \mathrm{fs}$.

- CHIMIE INORGANIQUE ET ORGANIQUE dang l'état actuel de la science, par M. PRRgradD. 1 gros volume orné de figures.

3 fr. 50 


\section{$-9-$}

MANUEL DE CHIMIE ANALYTIQUE, contenant des notions sur les manipulations chimiques, les éléments d'analyse inorganique qualifative et quantitative, et des principes de chimie organique, par MM. WILl, F. Voenler, J. Liebig et Malepeyre, 2 rol. ornés de planches et tableaux. $5 \mathrm{fr}$.

- CIDRE et POIRE (Fabricani de), avec les moyens d'imiter, avec le suc de ponme ou de poire, le Viry de raisin, l'Wau-de--Yie el le Viuaigre de vin, par M. DUBIEF. 1 vol. arec figures.

$2 \mathrm{fr} . \mathrm{bo}$

- CISELEUR, contenant la description des procédés de l'art de ciseler el repousser tous les métaux ductiles, bijouterie, orférrerie, armures, bronzes, etc., par MI. Jean GarNIER, ciseleur-sculpteur. 1 vol. orné de fig. $5 \mathrm{fr}$.

- COIFFEUR, précédé de l'Art de se coiffer soi-même, par M. Villaret, 1 joli vol. orné de fig. 2 fr. 50

- COLLES (Fabrication de toutes sortes de), comprenan! celles de matières végétales, animales et composées, par M. Malepeyre. 1 vol, orné de planches. 1 fr. 50

- COLORISTE, contenant le mélange el l'empló des couleurs, ainsi que les différents travaux de l'Enluminure, par MM. Perrot, Blanchard et Thillaye. 1 vol. 2 fr. 50 COMMERCE, BANQUE ET CHANGE, contenan! tout ce qui est relatif aux effets de Commerce, à la tevue des livres, à la comptabilité, à la bourse, aux emprunts, etc., par M. Gallas et M. Pijon. 2 vol. 6 fr. BONNE COMPAGNE, ou Guido ḋo la Politesse ot de la Bienséance, par Mme CrLINART. 1 vol. $1 \mathrm{fr} .75$ COMPTES-FAITS, ou Barême général des poids ể mesures, par M. AcHILle NovHen. (Toir Poids et Mlesures.) - CONSTRUCTEUR en GENERAL et AGENTSFOYERS, ourrage utile aux ingénieurs des ponts et chaussées, aux officiers du génie militairs, aux architectes, auz conducteurs des ponts et chaussées, par M. LAGARDE, in = génieur civil. 1 vol. orné de figures.

3 fr.

- CONSTRUCTION MODERNE (La), ou Traité de l'Art de bâtir avec solidité, économie et durée, comprenant la Construction, l'histoire de l'Architecture et l'Ornementation des édifices, par M. BATAILLE, architecte, professeur à l'école de Mulhouse, un volume avec 44 planches in- $4^{0}$ (sous presse).

- CONSTRUCTIONS RURALE, ou Guide pour les Constructions rurales, par M. Heuzi. 2 vol. $6 \mathrm{fr}$. - CONTRE-POISONS, ou Traitement des Individus 


\section{- $30 \ldots$}

empoisonnés, asphyxiés, noyés ou mordus, par M. H. VHAUSSIER, D.-M. 1 vol.

2 fr. 50

MANUEe DEs CONTRIBUTIONS DIRECTES, Guide des Contribuables et des Comptables de tontes les classes, etc.; Pa) BoYARD. 1 vol.

2 fr. 5 s

- CORDIER, contenant la culture des Plantes textiles, l'extraction de la Filasse, et la fabrication de toutes sortes Ee cordes, par M. BoitaRd. 1 vol. orné de fig.

2 fr. 30

- CORRESPONDANCE COMMERCIALE, contenant (os Termes de commerce, les Miodèles et Formules épistolaires et de comptabilité, etc., par MiM. Rers-Lestienne of TREMERY. 1 vol.

2 fr. 50

- CORPS GRAS CONCRETS. V. Bougies stéariques.

- COTON et PAPIER-POUDRE, voyez Allumettes chimiques

- COULEURS (fabricant de)ET VERNIS, contenant tout ce qui a rapport à ces différents arts, par MM. RifFAULT, VERGNAUE et TOUSSAINT. 1 vol. orné de fig. 3 fr.

- COUPE DES PIERRES, par MM. TOUSSAINT et H-M.M., architectes, 1 vol. a vec Atlas.

$3 \mathrm{fr}$.

- COUTELIER, ou l'Art de faire tous les Ourrages de Geutellerie, par M. LAND RIN, ingénieur civil. 1 vol. $3 \mathrm{fr} .50$ - CRUSTACES (Hist. natur. des j, par MM. Bosc et DES道A R

$6 \mathrm{fr}$.

ÁTLAS POUน LES CRUSTACÉS, 18 planchos. Figures noires. 3 ir.; - figures coloriées.

$6 \mathrm{fr}$.

- CUISINIER ET DE LA CUISINIÈRE, à l'usage de la vilie at de la campagne, par M. CarnosLly. I groe vol. do 46 s pages, orné de figures.

2 fr. 50

- CULTIVATEUR FORESTIER, contenant l'Art de sultiver enforête tous les Arbres indigènes et exotiques, par M. BOITARD. 2 volumes.

5 fr.

- CULTIVATEUR FRANCIAIS, ou l'Art de bienculiver les Terres et d'en retirer un grand profit, par M. THIEBAUT de BERREACD. 2 volumes ornés de figures.

- DAGUERREOTYPIE. Yoyez Pholographie:

- DAMES, on l'Art de l'Élégance, par madame NART. 1 vol.

5 fr.

CEL3 fr. - DANSE, comprenant la théorie, la pratique et l'hiztoire de cet art, par MM. Blasis of VERGNAUd. 1 gros solume orvé de planches.

3 fr. 50

- DECORATEUR-ORNEMENTISTE, du Graveur et du Peintre en Lettres, par M. ScharT, un vol, arec Atlas in-40 de 30 planches.

$7 \mathrm{fr}$. 
MANUEL DES DEMOISELLES, ou A rts et métiers qui leur conviennont, tels que Couture, Broderie, etc., par madame Cerratet. 1 rol. or né de planches.

$3 \mathrm{fr}$.

DESSIN LINÉAIRE, par M. A LLAIN, entrepreneur de travaux publics. 1 v. avec Allas de $20 \mathrm{pl}$.

$5 \mathrm{fr}$.

- DESSINATEUR, ou Traité complet do Dessis, par M. Bottencad. 1 v. avec Allas de $20 \mathrm{pl}$.

3 fr. 30

DISTILLATEUR ET LIQUORISTE, par M. LEBeAu et M. JULIA De FonTENELL. 1 rol. 18491 pages, orné de figures.

3 fr. 50 - DISTILLATION DE L'EAU-DE-VIE DE POMMES DE TERRE ET DE BETTERAYES, par MM. HOURIER et MALePeYRe, 1 rol. a pec fig.

1 fr. 50

- DOMESTIQUES, ou l'Art de former de hons Servin teurs, par madame CELNART. 1 rol.

2 îr. 30

- DORURE ET ARGENTURE par la méthode Electrochimique et par simple immersion, par MM. Malepeyre, Mathey et De Valicount. 1 vol. orué de fig. $1 \mathrm{fr}$. 80 - FABEILANY DE DRAPS, ou Traile de la Fubrication des Draps, par MM. BONNET el MALEPEYRe. 1 7ol. orvé de figures.

3 fr 30

- CCCLES PRIMAIRES, MOYENNES T NORMALES (Ouvrage autorisé par l'Université), par M. MATTER. 1 vol.

2 fr. 50

- ECONOMIE DOMESTIQUE, contenant toutez les raceiles le plus simples of les plus efficacez, par madame Crinart. 1 vol.

2 fr. 30

- ECONOMTE POLTIQUE, par B. J. Patet. 1 wol. Eir. 80

- ELECTRICITE, contenant les instructions pour établir les Baraton. et les Paragrèles, par M. RIE RALT.í v. 2 fr. 50 - ELECTRICITÉ MÉDiCALE ou Elément delectroBiologie, suivi d'un Traité sur la Vision, par M. SMeE, traduit par M. Magnier. 1 joli volume orné de fig. oै fr.

- ENCRES (Fabricat. de toutes sortes d'), soil pour l'éeriture, l'imprimerie, les encres sympathiques, etc., par MM. DE CGAMYOOR et F. MALEPEYR. $1 \mathrm{VOl}$. 1 fr.50

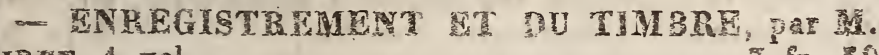
BIRET. I Tol.

$$
\text { sir. } 30
$$

- FNTOMOLOGIE ÉLEMENTAIRE, ou Eairetiens sur les Insectes es général, mis à la portée de soul le monde, par M. BOYeR de Fonscolome. 1 gros vol. $3 \mathrm{f}$. ENTOMOLOGIE, ou Hist. nat. des Insectes et des Myriapodes, pas M. BoiraRd. 3 rol. 10 fr. 30 
ATtas d'Entomologie, composé de 110 planches reprá sontant les Insectes décrits dans l'ouvrage ci-dessus. Figures noires, 17 fr. - Figures coloriées.

3.4 fr.

MANUEL DU STYLE EPISTOLAIRE, par MI BISCARMAT el adamo la comtesse d'HACTPOUL. 1 rol. 2 fr. 30

- ERUITATION, à l'usago de defix sexes, par M. FEnGNAUD. 1 vol, orné de gigures. $3 \mathrm{fr}$.

- ESCALIERS EN BOIS (Construction des), or manipulation et posatge des Escaliers ayant une ou plusieurs rempes, par C. Bovtencar. 1 vol. et Atlas.

3 fr.

- ESCRIME, ou Traité de l'Art de faire de armes, par M. LAFAUGkR, maréchal-des-logis. 1 pol. 3 fr. 30

- ESSAYEUR, par MM. VACQUELIN, GaY-Lossac et D'ARCET, publie par M. VERgRAUD. 1 vol. $3 \mathrm{fr}$.

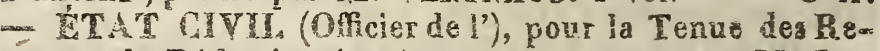

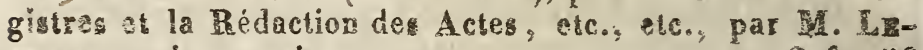
HOLT: ancien magistrat.

ใ fr. 30

- ETOFEES MPPRMES (Fabricantd') st Fabricam de Papiers peints, par MM. SÉB. LRTORMAND et VerGNAUD. 1 vol.

3 fr.

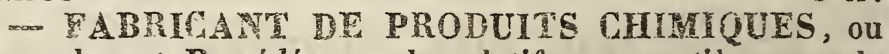
Formules et Procédés usuels relatifs aux matières çu la chimie fournit aux arts industriels et à la médecine, par

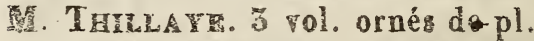

10 fr. 30

- ALSIFICATIONS DES DROCUES simple t compo-

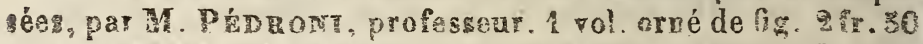

- WER HEANTER ET LAMPISTE, ou l'Art do confactionaer en fer-blanc toug les Ustensilea, par MM. LEpot at Mataperre. 1 vol. orné de fig.

3 fr. 50

- FERMIFR (du), ou l'Agriculture simplifiée el mise à la portée de tout le monde, par M. DE Lífinois. 1 vol. 2 fr. 30

ow FILATEUR, ou Description des Méthodes anciennes et noarelles employées pour filer le Coton, le Lin, le Chanvre, la Laine et la Soie, par MM. C.- E. JuLLIK et E. LOR

3 fr. 30

- FILATURE DE COTON, suivi de Formules pour apprécier la résistance des appareils mécaniques, elc., par M. Drapier, 1 volume arec planches, 2 fr. 50 - FLEULISTE ARTIFICIEL, ou l'Art d'initer, d'aprèz nature, toute espèce de Fleurs, zuivi de l'Art du Plamassier, parmadame CaLNART. 1 rol. orné de fig. 2 fr. 30

- FLEURISTE ARTIFICIEL SIMPLIFIÉ, par Mlle SOCRDON. 1 vol.

1 fr. 50

- FLEURS (des) EMBLEMATIQUES, on leur His. 
toirs, lear Symbole, leur Langage, etc., etc., par madame LENEYRUX. 1 vol. Fig. noires, 3 fr.; fig. coloriéss. $6 \mathrm{fr}$. A ANUEL DU FONDEUR SUL TOUS METAUX, par

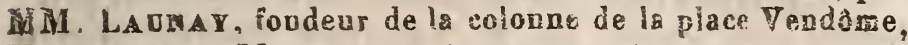

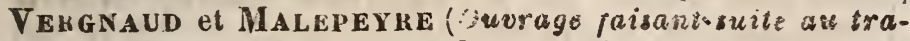
vail des aélauxi). zu vol. ornés d'vn grand nombre de pl. $7 \mathrm{fr}$. - FORGERON, MARECHAL, SERRURIER, TAILLANDIER, etc., renfermant des notions sur le fer, l'acier et les charbons; des modèles de forges, el vouvani servir de manuel complet du fabricant de soufflets et de ma. chines soufflantes, par M. MAPOD, 1 vol. orné de $4 \mathrm{pl}$. $3 \mathrm{fr}$.

- FORGES (Maître de), ou l'Art de travailler le reš, par M. LANDRYis. 2 vol. ornés de planches.

6 er

- FORESTIER PRATICIEN (le) et Guide des Gardes Champêtres, traitant de la Conservation des Semis, de l'Aménagement, de l'Exp!oitation, etc., etc., des Forêts, par MM. CRINon el VASSEROT. 1 vol.

if 25

GAL VANOPLASTIE, ou Traité complez do cet APE, contenant tous les procèdés les plus récents, par MM. SEER, Jacobi, urt Valicourt, etc., etc. 2 vol. orpés de fig. Bft.

- GANTS (Fabricant de) dans ses rapports a a la la gisserio et la Chamoiserio, par VAlLET D'derois, ancien fabricant. 1 rol.

Is. 30

- GARANTIE DES MITIERES DOR ET D'A GEPT, par M. LACHÈze, contrôlear \& Paris. 1 v. 1 fr. 75

- GARUES-CHAMPETRES, FORESTILIS RT GARUES-PECHE, par M. BoYARB, président à la cour d'sppel d'Orléave. ' тol.

2650

- GARDES-MALADES, ot perzonne sui ceulent se spigner ollos-mêmes, ou l'Ami de la santé, par M. lo chocteur WORYR. I VOl.

2. 5.50

GARDES NATIONAUE DE FRANCE, contenant I'Ecole du soldat et de pelotor, les Ordounances, Règlements, etc., etc., par M. Th. L. $33^{6}$ édit. 1 rol. $1 \mathrm{fr}$. 2o - GAZ (Fábrication du) ou Traité do lThelairage a

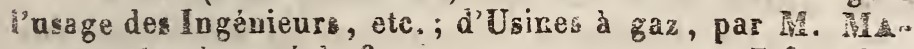
GMIRli. 1 vol orné de higures. $\quad 3 \mathrm{fr}$. 50 c. -GEOGRAPHIE DE LA FRARCR, divise par bassing. par M. LoRrou. (Autorisé par l'Univarsité). 1 rot. \& fr. 50

- GEOGHAPHIE GENERALE, par H. DEVILLIE. 1 gros vol. de plus de 400 pag., orné de 7 jolies cartes. 3 f.s. 0 - GEOGRAPHIE PHYSIQUE, ou Introduction à l'b. tude de la Géologie, par M. HUоT.1 rol.

3 iิ. 
MANUEL DE GÉOLOGIE, ou Traité élémentaire de cetto science, par MM. Heot ol D'CrbigNy. 1 p. orné de pl. 3 fr.

- GEOMÉTRIE, ou Exposition élémentaire de principes de cette science, par M. TKRQUE (Oxvrage autorise par l'Université). 1 gros vol.

3 fr. 50

- GNOMONIQUE, ou l'Art de tracer les cadrans, par M. Bovteread. 1 vol. orné de figures.

$3 \mathrm{fr}$.

- GOURMANDS (des), ou l'Art de faire les honneur: de sa table, par Cardelli. 1 vol.

3 ir.

- GRAVEUR (du), ou Traité complet de l'Árt de la GYavure en tous genres, par MM. BRRROT el MALEPEYRE. 1 vol. orné de planches.

$3 \mathrm{fr}$.

- GRECE (Histoira de la), depuís le premiers siècles jasqu'à l'établissement de la domination romaine, par

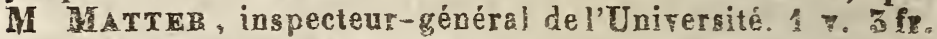

- GREFFES (Monographie des), ou Description des diverses sortes de Greffes employét's pour la multiplication des végétaux, par M. Thourx, de l'Institut, etc. 1 rol. orné de 8 planches.

2 fr. 50

- GUTTA-PERCHA, CAOUTCHOUC, etc. $3 \mathrm{fr} .50$

- GYMNASTLQUE (de la), par le colonel Amoros (Ouvrage couronné par l'Institut, admis par l'Universit', efs.). 2 vol.et Atlas.

10 fr. 30

- RABITANTS DE LA CAMPAGNE at Bonne Fermière, coutenant tous les moyens de faire valoir, de la mznière la plus profitable, les terres, le bétail, les récolter; etc., par madame Celnart. 1 vol.

- HÉraldique. Poyez Blasore.

- HERBoRISTE, Epicier-Droguiste, Grainier-Pépiniériste et Horticulteur, pas MM. TOLLARD et JulIA DE FONTERELE. \& gros Vol.

$7 \mathrm{fr}$.

- HISTOIRE NATURLLE, on Genera comple des A aimaux, des Végétaux el des Minéraux. 2 gros vol. " $\mathbf{f}_{\mathrm{B}}$

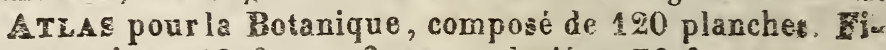
gures noires, $18 \mathrm{fr}$. - figures coloriées, $36 \mathrm{fr}$.

- pour les Mollusques, Eeprésentant le Mollusques nus et les Coquilles, 51 planches, figures noires, of figure coloriées.

14 ir.

- pour les Crustacée 18 planches, figures noires 3 francs - figures coloriées. $6 \mathrm{fr}$.

- Pour les Insectes, 110 planches, figures noires, $17 \mathrm{fr}$; figures coloriées.

34 fr.

- Pour les Mammifères, 80 planches, fig. noiren, 12 fr.; figures coloriées.

$24 \mathrm{fr}$. 
Atlas Pour les Minéraux, 40 planches, 8 gig. noires, 6 fr.; Ggures coloriées.

$12 \mathrm{fr}$.

- Pour les Oiseaux, 299 planches, figures noires, 20 fr.; figures coloriées.

- pour lez Poissons, 155 planchos, fing. noiros, figures coloriéez.

40 I.

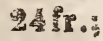

$48 \mathrm{fr}$.

- Pour les Repiles, 5s planchos, fig. noiros, \& fr. Gigures coloriées.

18 is

- Pour les Zoophytes, représentant la plupart dec Ters et des Animaux-Plantes, 85 pl., fgures noires, Ggures coloriées.

$6 \mathrm{fr}$.

MANUEL D'HISTOMRE MATURELE MYDCALE ET DE PRARMACOGRAPHIE, ou Tablean des Produit que la Médecine et les Arts empruntent à l'Histoire maturelle par M. LESSOR, pharmacien en chef de la Marine à Rochefort. 2 vol.

S IT.

- HISTOIRE UNIVERSELE, depuis le commencement du monde jusqu'en 1836, par M. CA do la Bible. 1 rol.

2 ir. 80

- HORLOGER (del'), or firuide des Ou rriers qui z'ocerpent de la construc. des Machines propres à mesurer le temps,

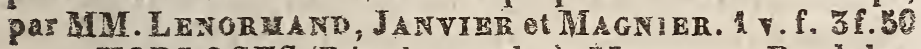

- HORLOGES (Rézulateur des), Montros et Peadules.

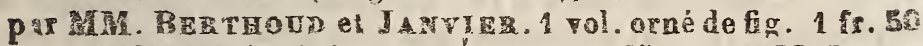

- HULLES (Fabricant et Épurateur d'), par MM. JULIA DE TOKTZNELLE et MALEPEYRE. 1 vol. ornd de fig. 3 ? 50 - HYGIENE, ou l'Art de conserver sa santé, par le docteur MoRIr. 1 rol.

3 is.

- INDIENNES (Fabricant d'), renfermant les Impresesions des Laines, des Chales et des Soies, par M. THILLAYE. 1 rol.

3 fr. 50

- INGENIEUR GIVIL, par JRP. JULLEN, LORETT ot Schmitz, Ingénieurs Civils. 2 gros rolumes arec 1 Allas renfermant beaucoup de planches.

10 fr. 30

- IRRIGATIONS ET ASSAINISSEMENT DES TERRES, ou Traité de l'emploi des Eaux en agriculturo. par M. le marquis DE PARTTo, 1 volumes oraés d'un atlas eomposé de 40 planches.

$18 \mathrm{fr}$.

- JARDINAGE (PRATIQUE SIMPLITIÉ E) al'usago des personnes qui cultivent elles-mêmes un petit domaine, conlonant un Polager, une Pépinière, un Verger, des Espaliers. un Jardin paysager, des Serres, des Orangeries, e! un Parterre, etc., par M. Louls DoßoIs. 1 vol. orné de fig. 2 fr. 50 
HANUEL DU JARDINIER, ou l'Art de cultiver et de composer toutes cortes de Jardins, par M. BAILcy. 2 gros vol. ornés de planches

5 fr.

- JARDINIER DES PRIMEURS, ou l'Art de forcer les Plantes à donner leurs fruits dans toutes les saisons, par MM. NoisetTr et Boitard. 1 vol. orné de figures. $3 \mathrm{fr}$.

- JARDINS (ART DE CULTIVER LES), renferman: an Calendrier indiquant mois par mois tous les travaux à faire en Jardinage, les principes d'Horticulture, etc.. par un Jardinier agronome. 1 gros vol. orné de fig. $3 \mathrm{fr} .50$

- JAUGEAGE ET DEBITANTS DE BOISSONS. 1 volume orné de figures (Voyez Vins). $\quad 3 \mathrm{ft} .50$ - JEUNES GENS, ou Sciences, Arts et Récréations qui leur conviennent, et dont ils peuvent s'occuper avec agrément et utilité, par M. VerGY AUD. 2 vol. ornés de fig. $6 \mathrm{fr}$.

- JEUX DE CALCUL ET DE MASARII, ou novvelle Académie des Jeux, par M. LEbrun. 1 vol.

3 fr.

- JEUX ENSEIGNANT LA SCIENCE, ou Introsuction à l'étude de la Mécanique, de la Physique, etc., par M. RiCHARD. 2 vol.

$6 \mathrm{fr}$.

- JEUX DE SOGIETE, renfermant tous ceur qui con viennent aux deuxsexes, par madame GeLNART. 1 rol. 3 fr.

- IISTICES DE PAIX, ou Traité des Compétences of Attributions tant anciennes que nouvelles, en toutes malières, par M. BrRet, ancien magistrat. 1 rol. $3 \mathrm{fr} .50$

- LAITERIE, ou Traité de toutes les méthodes pou la Laiterie, l'Art de faire le Beurre, de confectionner les Fromages, etc., par THIFBAUD DR BERNEAUD. i vol, orné de figures.

2 fr. 50

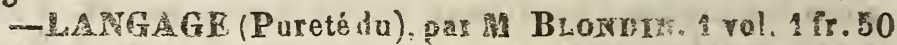

waNGAGR (Pureté du), par MM. BISGareat et BONIYACE 1 Tol.

a f 50

- LA TIN (Classes élémentaires de), ou Thêmes pour les Huitième el Septième, prar M. A médéc SEgIBR, ancien instituteur. 1 rol.

2 fr. 50

- MIMONADIER, Glacier, Chocolatier et Confiseur, par MM. Cardeli, Lionnet-Clémandot of Julia de Fontenelue. 1 gros vol. de plus de 500 pages. $3 \mathrm{fr}$.

- LITHOGRAPHE(Imprimeur), PAв MM. BREGEA चT, KNECHT et Jules DEsPORTES, 1 gros vol. a vec atlas. 5 fr. - LITTERATURE l'usage des deux sexes, par madame D'HAUtPoul.

1 fr. 75

- LUTHIER, contenant la Construction intérieure of 
extérieure des instruments à archets, par M. MAUGIN. 1 70lano.

2 fr. 50

MANUEL du Constructeur de MACINES LOCOMO. TIVES, pa) M. Jullier, Ingénieur civil, etc. 1 gros rol. arec Atlas.

Sร fr.

- MACHINES A VAPEUR appliquées d̀ la Marins. par M. JANrier, officier de marine et ingénieur civil. 1 voame arec figures.

3 fr. 30

- MACHINES 1 VAPEUR appliqué l'Industrò par M. JArvier. 2 rolumes avec figures.

$7 \mathrm{fs}$.

- MACON, PLATRIER, PAVEUR. GAREREIR, GUUVREUR, par M. TOUSSAINT, architecte. 1 70l. 5 fr. MAGIE NATURELLE IT AMUSANTE, par M. TERGAUD. 1 vol. avec figures.

3 if.

MAITRE D'HOTEL, ou Traité complel des menus, mis a la portée de tout le monde, par M. CFavileg. 1 vol. orné de figures.

$3 \mathrm{Ir}$.

MAITRESSE DE MAISON, par mesdames PARISET et CelNART. 1 rol.

2 fr. 50

- MAMMALOGIE, ou Histoire naturelle des Mammifères, pår M. LessoN, corresp. de l'Institut. 1 gros vol. 3 f. 30

Atras je uadualogie, composé de 80 planches Présentant la plupart des animaus décrite dans l'ourrage ci= lessus; figures noires, $12 \mathrm{fr}$; figures coloriées, 24 fr.

MARBRIER, CONSTRUCTEUR ET PROPRIETAIRE DE MAISONS, par MM. B. et M. 1 vol arec un bel atlas renfermant 20 pl. gravées sur acier.

$7 \mathrm{fr}$.

- MARINE, Grément, manceuvre da Ravirs et do l'Átillerie, par M. VERDIER, capitaine de corvette. 2 vol. ornés de figures.

$5 \mathrm{fr}$.

- MATHEMATIQUES (Applications usuelles at amugantes), par M. RICEARD. 1 gros vol. avec figures. 3 fr. - MECANICIEN-FONTAINER, SONDEUR, POMPIER ET PLOMBIER, par MM. JANvIzR, BISTON et MALEPEIRE. 1 vol. orné de plánches.

3 f. 50 - MECANIQUE, ou Exposition élémentaire des lois do 'Lquilibre et du Mourement des Corps solides, par M. THRQUEM, officier de l'Université, professeur aux Ecole royales d'Ártillerie. 1 gros vol. orné de planches. $3 \mathrm{fr}$. 30

- MECANIQUE APPLIQUEE A L'INDUSTRIE. Première partie. STATIQUE et HuDEOSTATIQUE, par M. VERGNAUD, 1 vol. avec figures.

3 fr. 50 - Deuxième partie, HYDRAdLIOJI, par M. JAN7IER. rolume arec figures. 
MANUEL DE MÉCANIQUE PRATIQUE, à l'usage des directeurs ts contre-maitres, par BERNodiLli, trad.par VALÉRIOS vol.

MEDECINE ET CHIRURGIE DOMESTIQUES, par . le docteur MoRiN. 1 vol.

$3 \mathrm{f} .50$

MENUISIER, Ébéniste, Layetier, Marqueteur et Sculpteur sur bois, par M. Nosban 2 rol. arec pl. $7 \mathrm{fr}$.

- MENUISERIE SIMPLIFIÉ, à l'usage des amateurs et des apprentis, par M. BouziQue. 1 vol. arec pl. 1 fr. 30

-METAUX (Travail des), Fer et Acier manufaciurés: par M. VERGNAUD. 2 Vol.

- MÉTREUR ET DU VÉRIFICATEUR EN BATIMENTS ou Traité de l'Art de métrer et de vérifier tous les suvrages en bâtiments, par M. LeBosso, architecte-expert.

Première partie. Terrasse et maçonnerie, 1 vol. 2 fr. 50

Deuxième partie. Menuiserie, peinture, tenture, vitrerie, dorure, charpente, serrurerie, couverture, plomberie, marbrerie, carrelagc, pavage, poêlerie, etc. 1 vol. $2 \mathrm{fr} .50$.

(Toyez Toiseur en batiments.)

- MICROSCOPE (Ouservateur au), par F. DUJARDrR, 1 vel. avec Atlas de 30 planches.

10 fr. 50

- EXPLOITATION DES MINES. Première partio: MOUILL (ou charbon de terre), par J.-D BLAFC. 1 rol. a vec figures.

3 i. 50

-Idem, 2 e partie, Fer, Plobs, Cervar. ETan, ARGBNT, OR, ZIRC, DIARANT, etc. 1 v. arec Âg. $3 \mathrm{f} .30$ - ART MILITAIRE, à l'usage des Militaires de soutes les armes, par M. VERGNAUD. 1 vol. orné de fig. 3 fr. - MiNERALOGIE, ou Tableau des Substances ming. rales, par $\mathrm{H}$. Hбот. 2 vol. ornés de figures.

$6 \mathrm{fr}$.

Ateas de Minéralogie, composé de 50 planches ro présentant la plupart des Minéraur décrite dans l'ourrage ci-dessus; figures noires.

Figures coloriées.

$6 \mathrm{fr}$,

MINIATURE, Gouache, Lavis à la Sépio et Aqua-

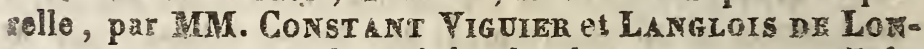
EDEVILLA. 1 gros vol. orné de planches.

3 ?

- MOLUSQUES (Histoiro naturelle des) et de leurs co-

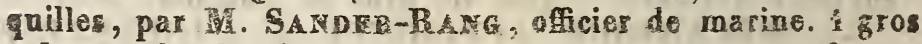
sol. orné de planches.

3 fr. 50

Atlas poUt las Mollusques, représentant le Molimsques nus et les Coquilles. 51 planches, fig. noires. 7 fr. Fig. coloriées.

$14 \mathrm{fr}$.

- MORALE, ou Droits et Deroirs dans la Société, 1 v. $75 \mathrm{c}$. 
MaNUEL DU MORALISTE, ou Pensées et Maximes instructives pour tous les àges de la vie, par M. TrzabiaY. 2 ro'umes.

$3 \mathrm{fr}$.

- MOULEUR, ou l'Art do mouler en plàtro, carton, arton-pierre, carton-cuir, cire, plomb, argile, bois, écaille. sorne, etc., par M. LEBron. 1 vol. orné de fig. 2 fr. 30

- MOULEUR EN MEDAILLES, otc., parM.RoBERT. 1 rol. avec figures.

$1 \mathrm{fr} .5 \mathrm{C}$

- MUNICIPAUX (Officiers), on Nouvean Guido des Maires, Adjoints et Conseillers municipaux, par M. BorABD, président à la Cour d'appel d'Orléans. 1 gros vol. $3 \mathrm{~h}$. 50

- MUSIQUE, ou Grammaire contenant les principes de set art, par M. LED'HUY. 1 v. avec 48 pages de musiçae. 1 f. 3

- MUSIQUE VOCALE ET INSTRUMENTALE, о Incjclopédie musicale, par M. ChoroN, ancien directeur 10 l'Opéra, fondateur du Conservatoire de Musique elassisque et religieuse, et M. DR LAFAGR, professeur de chent et I $^{2}$ composition.

\section{ATISIOR DE E'OUPTBEE \\ IP PARTIB. - RXZCOTIOT.}

LIVRE I. Connaissances élémentaires. Sect. 1. Sons, Notations.

2. Instruments, sxécution.

$\left\{\begin{array}{c}1 \text { rolukes } \\ \text { spes Atsas }\end{array}\right\}$

s?.:

\section{ALTIE.- COMPOSITIOW.}

- 2. De la composition er géréral, et

- 3. Del'Harmonie.

- 5u Contre-Point.

5. Imitation.

- E. Instrumentation.

- F. Union de la sinsiqqu arer la

Parole.

Parol

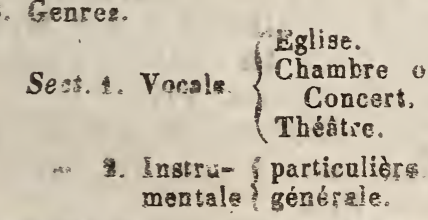

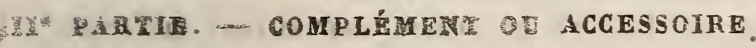

- 9. Théorie physico-mathématigue.

- 10. Institutions.

- 11. Histoire de la musique

- 12. Bibliographie.

ßésumé générø!.

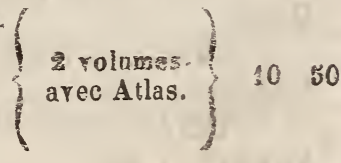


SOr. ĖGEs, MÉTHODE.

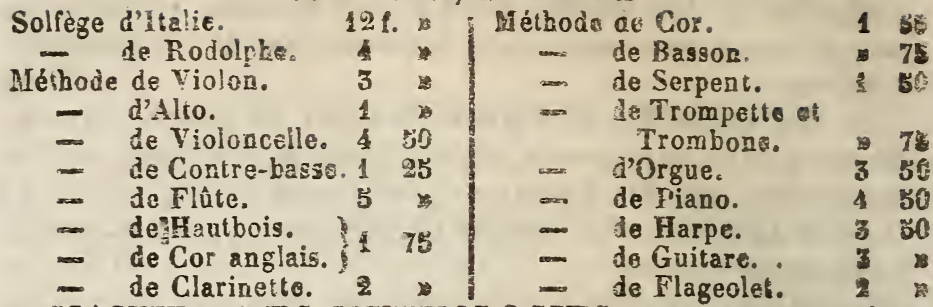

MANUEL DES MYTHOLOGIES grecque, romaino, égyptienne, syrienne, africaine, etc., par M. DuBors. (Ou. rrage autorisé par l'Université.) 1 vol.

2 fr. 30

- NAGEURS, Baigncurs, Fabricants d'eaux minérales et des Pédicures, par M. JULIA DE FONTENELLE. 1 vol. 3 fr.

- NATURALISTE PREPARATEUR, or 'Art d'empailler les animauz, de conserver les Végétaus et les Minézaux, de préparer les pièces d'Anatomie et d'embaumer, par M. BoitaRD. 1 rol. arec figures.

3 fr. 50

- NAVIGATION, contenant lá manière de se servir de l'Octant et du Sextant, de rectifier ces instruments et de s'assurer de leur bonté ; l'exposé des méthodes les plus usuelle d'astronomie nautique, pour déterminer l'instant de la pleine mer, etc., etc., et les tables nécessaires pour effectuer ces différents calculs, par M. GIQUEI, professeur d'hydrographie. 1 rolume orné de figures.

2. fr. 30

- NAYIGATION INTERIEURE, à l'usage des Pilotes, Mariniers ef Agents, ou Instructions relatives aux devoirs des mariniers et agents employés au service de la navigation intérieure, par M. BEA UVALET, inspecteur de la navigation le la Basse-Seine. 1 vol.

2 fr. 50 -NEGOCIANT D'EAI-DE-VIE, Liquoriste, MIarchand de vin et Distillateur, par MM. Rayon et MALEPEYRE, 1 r. $75 \mathrm{c}$.

- NUMISMATIQUE E NCIENNE, par M. BartuÉLEHY, ancien élève de l'Ecole des Chartes. 1 gros rol. orné d'un Atlas reofermant $4 \bar{j}$ figures. Frix

- NUMISMATIQUE MODERNE ET DU MOYEN$\triangle G E$, par M. BARTEÉlEMY. 1 gros vol. orzé d'un Atlas renfermant 12 planches. Prix

5 fr.

- OCTROIS et qutres impositions indirectes, par $\mathbf{M}$. Broze. 1 rol.

3 fr. 50

-OISELEUR (Do l'), ou Secrets anciens et modernes do la Chasse aux Oiseaux, par MI. J. G., 1 vol. orné de fig. 2 fr. 50 - ONANISMIE (Dangers de l'), par M. DodsSIN-DoBREUIL. 1 rol. 
MANOEL DOPTIQUE, on Traité complet de cette acionco, par BREWSTER et VERGRAUD. 2 $v$. avec fig. $6 \mathrm{fr}$

- ORGANISTE-PRA TICIEN, contenant l'histoire de l'orgue, sa description, la manière de le jouer, etc., par M. Georges SchMitr, organiste de St-Sulpice. 1 vol. orné de figures et musique.

2 fr. 50

- ORGANISTE, ou Nouvelle Méthode pout exécuter sur l'orguo tous les of fices de l'année, etc., par 塑. Mrr. organiste à Saint-Roch. 1 rol. oblong.

$3 \mathrm{fr} .3 \mathrm{~s}$

- ORGUES (Facteur d'), contenant le traiail de DoM Bédos, etc., etc., par M. HACEL, juge à Bearvais, 3 Fol. arec un grand allas.

- ORNEMENTISTE. Voyez Décorateur.

- ORNITHOLOGIE, w Description des genreg 8 की principales espèces d'oiseaux, par M. LAESOR, correspondent de l'Institut. 2 grns vol.

7 f.

AtLas D'ORnithologie, composé de 129 planches ro. présentant les oiseanx décrits dans l'ourrage ci-dessus; figu ros noires, $20 \mathrm{fr}$; figures coloriées.

$40 \mathrm{fs}$.

- ORNITHOLOGIE DOMESTIQUE, on Guido do l'Amateur des oiseauy de volière, par M. L ESSON, correspoudant de l'Institut. 1 vol.

$2 \mathrm{fr} .56$

- ORTHOGRAPHISTE, ou Cours théorique ef pratique d'Orthographe, par M. Tre

- PAlÉontologie, ou des lois de l'organisation des êtres viranta comparées à celles qu'ont auivies lss Espèces fossilez et humatiles dans leur apparition successi :par M. MARcel DE SRRres, professeur b la Faculté des Sciences de Miontpellier. 2 vol., avec AlIas.

$7 \mathrm{fr}$.

- PAPETIER ET REGLEUR (Marchand), par MM. JU. LIA DB FonTENeLLget PoISsors. 1 gros y. avec pl. $3 \mathrm{fr} .50$

- PAPIERS (Fabricant de), Carton et Art du Formaire. par M. LENORMAMD. 2 vol. et Atlas., 10 R. BÉ

- PAPIERS DE FANTAISIE (Fabricant de), $\mathbf{P a}_{\mathbf{2}}$ piers marbrés, jaspés, coaroquinés, gaufrés, dorés, esc.; Peau d'âne factice, Papiers métalliques; Cire et Pains à cacheter, Crayons, elc., etc.; par M. FICHTENBerg. 1 rol. orné de modèles de papiers. Prix

$3 \mathrm{fr}$

PAR FUMEUR, par Mme CElrast. 1 rol. 2 fr. 30

- PARIS (Voyageur dans), on Guide dans cetts capitale, par M. Labrun. 1 gros vol. orné de fig. 3 fr. $g$ - BARIS (Voyageur anx onvirong de), par D. DIRAT. 1 vol. arec figures.

5 tr. 
MIFULL DU PATINAGE et Récréations sur la Glace, par M. Paulin Désormeaux. 1 y. orné de $\$ \mathrm{pl}$. $1 \mathrm{fr} .25$

- PATISSIER ET PATISSIERE, ou Traité complet et simplifié de Pâtisserie de ménage, de houtique et d'hôtel, par M. Leblanc. 1 rol,

a fr. 50

PATISSERIE LEGERE, vojez PetrT-Four.

- PECHEUR, ou Truité général de toutes sorter de gêshes, par M. Pesson-Maisonneuvio 1 vol. orné de pl. $3 \mathrm{ir}$.

- PÉCHEUR-PRATICIEN, ou les Secrots of Mystè-. res de la Pêche dévoilés, parM. LABBERT, amateur; suiv de l'Art de Paire des filets. 1 joli vol. orné de fig. 1 fr. 75

- PEINTRE D'HISTOIRE ET SCULPTEUR, OUvrage dans lequel on traite de la philosophie de l'Art et des moyens pratiquee, parM. AnSENre, peinire. 1 rol. 3 fy 50 - PEINTURE a LAQUARELLE (Cours de), pas M. P. D., un vol. orné de planches coloriées. $1 \mathrm{fr}$. 75 - PEINTRE EN BATRMENTS, Vitrier, Doreur, argentaur et Vernisseur, par MM. RIFFAULT, VERGNADD ot ToussaINT. un yol. orné de figures.

$3 \mathrm{fr}$.

- PEINTURE ET FABRICA TION DES COULEURS, ou Traité des diverses Peiniures, à l'usage des deux sexes, par M. Joseph Panien, élève et successeur de M. LaMBERTYE, fabricant de couleurs fines, etc., 1 vol. $1 \mathrm{fr} .50$

- PEINTURE SUR VERRE, SUR PORCELAINE IN SUR SMAIL, contenant la Théorie des émauz, etc., par M. Reboullead. 1 vol. avec figures.

2 fr. 50

- PERSPECTIVE, Dessinateur et Peintre, par M. VERGNAUD, chef d'escádron d'artillerit. numbre de plancher.

3 fs.

- PETIT-FOUR, ou Pâtisserie légère, par $M$. Antoine Gross. 1 vol.

2 fr. 50

- PHARMAGIE POPULAIRE, simplifiée et mise à la portée de toutes les classes de la société, par M. Julia DE Fontenelie. 2 vol.

- PHILOSOPHIE EXPERIMENTALE, à l'usage des collèges et des gens du monde, par M. All ICE, régent dans l'A cadémie de Paris. 1 gros rol.

3 fr. 50

- PHOTOGRAPHIE surMéial, sur Papier et sui Verre, contenant toutes les décourertes les plus récentes dan: la Daguerréotypie par M. DEVALICo onT. 2 v. ornés de fig. $6 \mathrm{f}$.

- PHOTOGRAPHE (GUIEE DU), ou l'Art pratique et théorique de faire des Portraits sur Verre, Papier, Métal, etc., etc., au moyen de l'action de la lumière, par MM. J. Sella et de Valicourt. 1 gros vol.

3 fr. 50 
MANUEL DE PHOTOGRAPHIE (Simplifié) sur verre et sur papier, par M. DE LATREILLE. 1 vol. $1 \mathrm{fr} .50$ - PHOTOGRAPHIE (Repertoire de), par M. DE LATREILLE.

3 fr. 50

- PHYSIOLOGIE VÉGTALE, Physique, Chimio et Minéralogie appliquée à la culture, par M. MoITARD. 1 rol. orné de planches.

$3 \mathrm{fr}$.

- PHYSIONOMISTE ET PHRENOLOGISTE, ou les Caractères dévoilés par les signes extérieurs, d'après Lavater, par MM. H. Guadssief fils et le docteur Momin. \$ rol. arec figures.

$3 \mathrm{ft}$

- PIXSIONOMISTE DES DAMES, d'aprèz Lavater, par un Amateur, 1 vol. arec figure

$3 \mathrm{fr}$. PHYSICIEN-PRÉPARATEUR, or nouvelle Description d'un cabinet de Physique, par MM. Ch. ChervaLIER el le Dr FAc. 2 gros vol. arec un Allas de $88 \mathrm{pl}$. $15 \mathrm{fr}$.

- PHYSIQUE, ou Eléments abrégés de cette Ŝcience mise à la portèe des gens du monde et des étudianals, pas MAILEY, 1 7ol. avec figures,

2 fr. 30

PHYSIQUE APPLIQUEE AUX ARTS ET METIERS, principalement à la construction des Fourneaux, des Cáiorifères, des Machines à vapeur, des Pompes, l'Ar du Fumiste, l'Opticien, Distillateur, Sècheries, Artillerie vapeur, Eciairage, Bélier et Presse hydrauliques, Aréomètres, Lampe à niveau constant, etc., par MM. GuIllocd ef Terrien. 1 volume orné de figures. 3 fr. 50

- PHYSIQUE AMUSANTE, ou Nouvelles Récréation: physiques, par JULU DP FONTERELLE. orvé de pl. $3 \mathrm{fr} .50$

- PLAIN-CHANT ECCLEEIASTIQUE, romain et trançais, par M. Mrvé, organiste à St-Roch. 1 vol. 2 fr. 30

- POELIER-FUMISTE, indiguant les moyens d'empê her les cheminées de fumer, do chaufer économiquemen: of d'aérer les habitations, les ateliers, etc, par MM. AR DEFNI Et JVLIA DE FONTENELLE. 1 gol. 3 ft. $5 \mathrm{C}$

POIDS ET MESURES, Monnaies, Calcul décimal et Vérification, par M. TARBE, conseiller a lo Cour de Cassation; approuvé par le Hinistre du Commerce, l'Université, la Société d'Encouragement, etc. 1 rol.

$3 \mathrm{fr}$.

- POIDS ET MESURES (Fabrication des), contenant en général tout ce qui concerne les Arts du Balancier et du Potier d'étain, et seulement ce qui est relatif à la Fabricatior. des Poids et Mesures dans les Arts du Fondeur, du Ferblantier, du Boisselier, par M. Ravor, vérificałeur au bu* reau central des Poids et Mesures. 1 vol. orné de fig. $3 \mathrm{fr}$. 
Patit Manoel à l'usage des Ouvriers et des Écoles; avec Tables de conversions, par M. TARPÉ.

25 c.

Petit Maneel classique pour l'enseignement élémen-

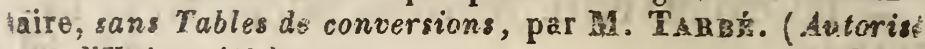
(Ior l'Universite.)

$25 \mathrm{c}$ :

Petrt Mantiel à l'usage des Agents Forestiers, des Propriétaires et Marchands de bois, par M. TARBí. $75 \mathrm{c}$.

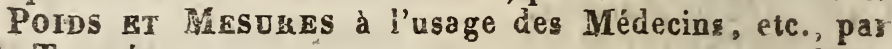
M. TARBí.

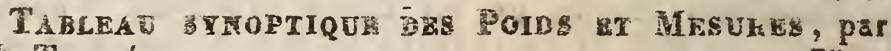
M. TARBí. $75 \mathrm{c}$.

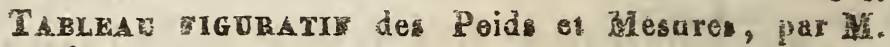
TaBbí.

75.

MANUEL DES POIDS RT MESURES, Eanue fomptos faits, ou Barême général des Poids et Mesures, par M. Acarle NovHen. Ouvrags divise an cing partiee qui se ven dens toutes séparément.

1 Po partio: Mesurez de LOMGUEUR.

is partie, $-m$ de SURFACE.

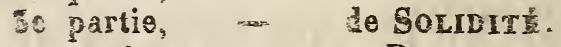

季 partie, - Forns.

: partie, - de CAPACIT扂.

30 . So $60 \leqslant$ 80. 50 ..

- POLICE DE LA FRANCE, par 翟. 管R sire äe police à Paris. 1 vol.

$2 \mathrm{ft} .50$

- PONTE ET CHAUSSEE3 : première partie, RoNTES CHEMINS, par M. DE GAYTFIRE, ingénieur do Pouts et Chaussées. 1 7ol. avec îg.

$3 \mathrm{fr} .5 \mathrm{~s}$

- Seconde partie, contenant les EorTs, 1 rolume avec figutes.

3 fr. 30

- Troisième partie, contenant LES EAUX DANS LEUR APPLICATION COMME FORCE MOTHCE AUX USINES UYdRAULiques, par M. GeNtilhomie, archilecle, 5 fr. 50

-.- PORCELAINIER, Faiencier, Fotier de serte, Briquetior of Tuilier, contenaat des notions pratiques sur la fabrication des Porcelaines, des Faïencen. des Pipes. Pnêles, deg Bricues, Tuiles et Carreaux, par M. Boyen. Nouv. édit. très-augmentée, par M. B... . Q. vol. ornés de pl., 6 fr.

- BRATICIEN, ou Traité de la Science du Droib, mise à la poriée de tout lo maonde, par MM. D.... ô RoNDONThAU. 1 gros vol.

$3 \mathrm{fr} . \mathrm{BS}$ - PRATIQUE SIMPLIFIEE TU JARDINAGE! $\mathrm{V}$. Jarảinage).

- PROPRIÉtAIRE ET LOCATAIRE, ou Sous- 
Locataire, tant des biens de ville que des biens ruraar, par M SERGENT. 1 rol.

$2 \mathrm{fr}, 30$

MANUEL DU RELIETR dans toutes ses parties, contenant les Arts d'assembler, de zetiner, de brocher et de dorer, par W. Seh. Lanor AND et M. R. 1 grosy. orné de pl. 3 fr.

- AMATEUR de ROSES, leur Monographis, loas Fistoire et leur Culture, par M. BortaBD. 1 rol. êg. noires 5 ir. 50 c., - et fig. coloriées.

7 in.

- SAPEUR-POMPIER, on Théorio an l'extinction des Incendies, par M. PAuliN, commandanidos SapeurePompiers de Paris. 1 vol.

1 fr. 30

ATLAS composé de 50 planches, faísani connâtro les machines que l'on emploie dans ce service, la disposition pouz attaquer les feux, les positions des Sapeurs dans toutes los manceny yres. etc.

ir.

- SAPEUR-POMPIER, currage composé par le corps des Officiers formant l'ètat-major, publié par ordre du wistre de la Guerre. 1 joli volumo renfermant une foulo do gravures sur bois imprimées a vec lo texte. Prix. 5 fr.

SAPEURS-POMPIERS (Théorie des), exirail du Manuel du Sapeur-Pompier, imprime par ordre du Ministre ds la Guerre.

$7 \mathrm{~s}$.

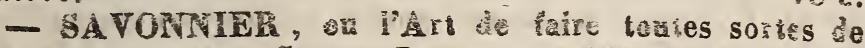

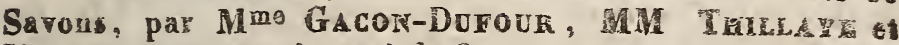
MALEPEYRE. 1 pol. orné de frg.

3 ใิ?

- SERRURIER, ou Traite complet ot zimplifé a coi Art, par MM. B. et G., serruriers, at Paulin-DisonMEAUX. 1 volumo orné de planches.

\% Ir. 30

- SOIERIE, contenans l'Art d'élever les Vers à soie ol do cultiver le Mûriér; l'Histoire, la Gréographio et la Fabrication des Soieries, à Lyon, ainsi que dans les autrea low calités nationales at étrangères, par M. DrYuLITERs. 2 velumes et A tias.

10 fr. 30

- 3OHMELIER, ou la Manière de zoignes les Tins, par M. JeLiefr. 1 vol. avec figures.

- SORGELLERIE ANCIENNE ET MODERNE EXPLIQUEE, ou Cours de Prestidigitation, contenant tous les Tours nouveaux qui ont été exéculés jusqu'à ce jour (1858) sur les theâlres ou ailleurs, et qui n'ont pas encore été publiés, etc., etc., par M. Ponsin, ancien professeur. 1 gros rolume.

$5 \mathrm{fr} 30$

- Supplément a la Sorcellerie expliquée, par M. Ponsin. 1 rol.

$1 \mathrm{fr} .25$ 
MANUEL DES SORCIERS, ou la Magie blanche dévoiléc par les découvertes de la Chimie, de la Physique et de la Mécanique, par MM. Comte et Julia DE FonteNELlE. 1 gros vol. orné de planches. 3 fr.

- SOUFFLEUR A LA LAMPE ET AJ CHALUMEAU, par M. PÉDRoñ, profes $r$ de chimie. 1 vol. orné de fig. 2f. 50 - FABRICANT DE SUCRE ET RAFEINEUR, par MM. BLACHETTE, ZOÉG et JULIA DE FONTENELLE. 1 vol. orné de figures.

3 fr. 50

- STENOGRAPHIE, ou l'Art de suivre la parole en écrivant, par M. M. Prívost. 1 vol.

$1 \mathrm{fr} .75$

- TABAC (Fabricant et Amateur de), contenant zon Histoire, sa Culture ot sa Fabrication, par P. Ge. Jordent. 1 rol.

2 fr. 50

- IMPRINETR EN TAILLE-DOUCE, par MM. RERTEIAUE et BotTaRD. 1 rol. avec fig.

$3 \mathrm{fr}$.

- TAILLEUR D'HABITS, contenant la manièro de tracer, couper et confectionner les Yêtements, par $\mathbf{M}$. VANDAEE, tailleur. 1 vol. orné de pl.

2 fr. 50

- TANNEUR, Corroyeur, Hongroyeur el Boyaudier, par M. JULIA DE FoNTENELLE. 1 vol. avec fig. 3 fr. 50

- TAPISSIER, Décorateur et marchand de Meubles, nar M. GARNIER ADDIGER, ancien vérificateur du GardeMeuble de la Couronne. 1 vol. orné de fig.

2 fr. 30

- TÉLERAPHE-ELECTRIQUE, ou Traité de l'Electricité et du Magnétisme appliqué i la transmission des siggaux, pat MM. WALZER et MAGNIEE, un vol. orne de Qgures.

1 ir. 75

- TEFEUP IDE LIVRES, renfermani an Cours de Ienue de Rivres a partie simple et à partie double, par

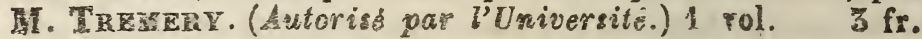

- TEINTURIER, contenant l'Art de Teindre en Isaine, Sole, Coton, Fil, otc., par M. VarGradd. 1 groz rol. avec figures.

$3 \mathrm{fr}$.

- TERRASSIER, par MM. ETIENA el MASSON, un vol. orré de 20 planches.

3 ir. 50

- THEATRAL el du Comédien, contenant les principes sar l'art de la parole, pat M. Aristippe Bernier DR MAPIGNY. 1 rol.

$3 \mathrm{fr} .50$

- TISEERAND, ou description des procédés el masinines employés pour les divers tissages, par MM. LoRENTz " JELIIEN. 1 vol. orné de fig.

3 ir. 50

- TISSSUS (Dessin et Fabrication des) façonnés, tels que Draps, Veloura, Ruban, Gilet, Coulil, Schall, Passe- 
menterie, Gazes, Barrèges, Tulle, Peluche, Damassé, Mousseline, etc., par M. Toustain, 2 vol. avec allas renfermant 26 planches in-4.

$15 \mathrm{fr}$.

MANUEL DU TOISEUR EN BATIMENT; $1^{\text {re partis: }}$ Terrass -et Maçonnerie, par M. LEBosSす, architecte-8Xpert. 1 v. arec fig. Voyez Métreur en batiments. 2 fr. 30

- Deuxieme partie : Menuiserio, Peinture, Tenture, Vitrerie, Dorure, Charpente, Serrurerie, Couverture, Plore. berio, Marbrerio, Carrelagø, Pavage, Poêlerie, Fumi terie, etc., par Mi. LEBosSd. 1 vol.

2 fr. 30

- TONNERIER ET BOISSELIER, zuivi \&e l'Art de faire les Cribles, Tamis, Soufflets, Formes et Sabots, par M. Désorimedux. 1 vol. àvec fig.

- TOURNEUR, ou Traité complet et simplifí de cet Art, d'aprè les renseignements de plusieur: Tourneurs do la capitale, par M. DE VALICOJRT. 2 Vol. arec un atlas in-40 de $25 \mathrm{pl}$.

$12 \mathrm{fr}$.

- TREIGLAGEUR ET MENUISIET DES IARDINS, par M. DÉSORMEA Ux. 1 rol. avec pl.

3 然

TYPOGRAPHIE, IMPRIMERIE, par MM. IREY et BөUCHEZ. 2 vol. avec planches.

6 ir.

- VERRIER ET FABRIGANT DE GLACES, Cristaux, Pierres précieuses factices, Verres coloriés, Yeux artifictels, par M. Julia DR ForTfrello et MALEPEYRE. 2 vol. ornés de planches.

6 f:. - VÉTERINAIRE, contenant la connaiszanco des chevaux, la manière de les élever, les dresser et les conduire; la Description de leurs maladies, les meilleurs modes de Iraitement, etc., par M. LaBEAU ot un ancien professeur d'Alfort. 1 rol. avec planches.

$3 \mathrm{fr}$.

- VINS DE FRUITS (Fabrication des), contenan! l'art de faire le Cidre, le Poiré, les Boissons rafraîchissantes,

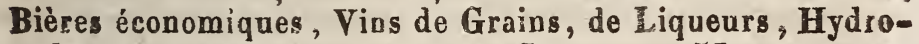
mels, etc., par MM. AccuM, GUIL.. et MALspiris, rol.

1 fr. 80

- VIGNERON FRANGAIS, on l' Vigne, de faire les Vins, les Eaux-de-Vie et Vinaigres, par

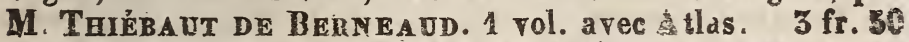

- VINAIGRIER IT MOUTARDIER, par A. JuLA DE Fontenelle. 1 vol. asec planches.

$2 \mathrm{f}$.

- VINS (Marchand de), débilant de Boigsons Jazgeage, par M. LAJDIRR. 1 yol avec planches. $3 \mathrm{f}, \mathbf{3 0}$ - ZOOPHILE, ou l'Art d'élever et do soigner los animaux domestiques (vovex Bourier). 1 vol. 2 fr. 50 


\section{BELLE ÉDITION, FORMAT IN OCTAVO.}

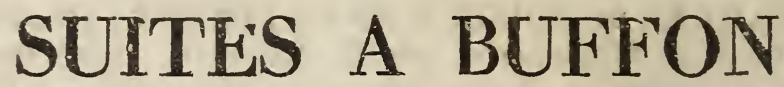

CORMANT,

AVEG LES OEUVRES DE CET AUTEUR,

US COURS COMRLET

D'RIS管OIRE NATURHE

embrassant

LES TROIS RÈGNES DE LA NATURE.

Les possesseurs des OEurres de BUFFON pourront, arec ses suites, compléter toutes les parties qui leur manquent, chaque ouvrage se vendant séparément, et formant, tous réunis, arec les travaux de cet hommo illustre, un ouvrage gẻnéral sur l'hisioire naturelle.

Cette publication scientifique, du plus haut intérêt, préparée en silence depuis plusieurs années, et confiée à ce que I'Institut et le haut enseignement possèdent de plus célèbres naturaiistes et de plus habiles écrivains, est appelée à fairo époque dans les annales du monde savant.

Les noms des Auteurs indiqués ci-caprès, sont, pourle public une garantie ceriaine de la conscience et du talent apportés a ia rédaction des différents traités.

TOOLOGIE GENERALE (Supplément à Buffon), ou עémoires et notices sur la 300 logie, l'anthropologie et l'histoire de la science, par 1. ISIDORE GEOFFROYSaikt-Hilaire. 1 volumoavec Atlas. Prix : fig. noires.

3 fr. 50 Sigures coloriées. 12 fr.50. CÉTACÉS, Baleiaes, DAUPHINS, otc.), ou Recueil et examen des faits dont se compose l'hisioire do ces znimaux, par M. $\mathbf{F}$. CeVIER, membre de l'Institut, professeur au Muséum d'Histoire naturelle, etc. rol. in.-8 avec 22 planches (Ouvrage terminé), figures noires. 12 fr. 50 Fig. eolorièes. $18 \% 50$ REPTILES Sorpout: Lézards, Grenouilles, Tor- 
tues, atc.), par M. DUM: RIL, membre de l'Institut, professeur à la faculté do Médecino et au Muséum d'Histoire naturelle, et $\mathbf{M}$. 蛋IBRON, professeur d'Histoire naturelle, 10 vol. of 10 livraisons de planches, fig. noires.

Tig. coloriẻes.

$95 \mathrm{fr}$.

(Ouvrage termivé.)

POISSONS, par M.

ENTOMOLOGIE (Introduction à l'), comprenant les principes généraux de l'Aanatomie et de la $P$ hysiologio des Insectes, des détails sur leurs mœurs, et un résumé des principaux systèmes de slassification, etc., par M. LACORDAIRE, doyen do la faculté des sciences à Liège (Ouvrage terminé, adoptś st recommandé pas l'Unizorsité pour être placé dans les bibliothéques des Faculbés at des Collèges, et donne en prix aux ślèves). 2 vol. in-8 et 24 planshes, fig. noires.

Fig. coloriées. $19 \mathrm{fr}$.

INSECTES

COLEOPTE-

RES (Cantharides, Charauçons, Hannetons, Gsa-

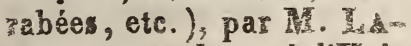
CORDAIRE, dOYen कl'URiversité de Liège.Tomes ${ }^{\circ}$ or, 20, $3^{\theta}$ at $4^{\circ}$.

$26 \mathrm{fr}$.

- ORTHOPTĖRES (Grillons, Criquets, Sauterelles), par M. SER vILLE, eX-pré sident de la Société entome. logique de France. 1 vol. et $14 \mathrm{pl}$. (Ouvrage termini). fig. noires. 9 fr. 50 c., et fig. coloriées. 12 fr. $50 \mathrm{c}$.

MÉMIPTĖRES (Cigales, Punaises, Cochenilles, etc.), par MM. AMYOT et SeRVILLE. 1 vol. et une livraison de pl. (Ouv. terminb.) Fig. noires. 8 fr. $50 \mathrm{c}$. Tit fig.coloriées. 12 fr.50: LEPIDOPTERES (Papillons), par MM. BoISDE-

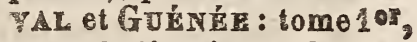
- avec 2 livraisons do ploğ tom. 3 à 10, avec 5 lis do pl. Fig. noire». 66 fr. 50 Fig. coloriéez. 87 ir. 50 NÉVROPTĖRES (Do.moiselles, Éphémères, etc.)s par M. le docteur RA 1 vol. arec une livraison de planches. (Ouvraga termsne). fig. noires $9 \mathrm{fr} .50 \mathrm{c.}$, et fig. coloriées 12 fr. 30 e.

HYMENOPTERS (Abeilles, Guêpes, Fourmis, etc.), par M. le comte IAPELETIEI DE SAIBT TARGEA et M. BRÚlLi ? 4 vol. avec livraisons de planches. (Ouv, terminé.) Fig.noires. Fig. coloriées. 38 fr. 30 tr. DIPTE'RES (Mouches, Cousins, etc.), par M. MAGQDABT, directeri du Muséum đ'Tistoire naturelle de Bille; 2 Tol. of 24 planches. (out. termind.) 
Big. noires.

丕ig. coloriẻes.

APTERES (Araignées, Scorpions, etc.), par M. WALGKGHER et le dosteur GERVAIS; rol. avec 5 cahiers de pl. (Ouv. term.) Fig. noires. $4 \mathrm{f}$. Fig. coloriées. CRUSTACES

$36 \mathrm{fr}$. (Écrevisses, Homards, Crabes, etc.), comprenant l'Anatomie, la Physiologie et la Classifi cation de ces animaux, par M. Milne - EDwards, ambre do l'Institut, etc. (Ouvrage terminé), 3 rol. arec 4 livraisons de pl. fig. noires.

31 fr. 50

Fig. coloriées. $\quad \mathbf{3}$ fr. 50 MOLLUSQUES' (Moules, Iuîtres, Escargots, Limaces, Coquilles, etc.), par M. DE BLainville, membro de l'Institut, professeur aa Muséum d'Histoire natarelle, etc.

AELMINTHES, ou Vers intestinaux, par M. DUJARDIr, do la Faculté des Seiences de Rennes. 1 vol. avec une livraison de pl. (Ouvrage terminé). Prix : fig. noires, $9 \mathrm{fr}$. 50 , t $\mathrm{fg}$. celoriées, 12 fr. 50 . ANNELIDES etc.), par $\mathbf{M}$.

Z00PEYTES CALEP FIS (Physa!e séroé, Angèle, atc.) par séc. LESsoN, correspondant de l'Institut, pharmacien ex chef de la Marine, à Rochefort, 1 rol. avec 1 livraison de pl. (Ouvrage termine.) fig. noires. 9 fr. 50 Tig. coloriées. 12 ir. 50 QCHINODERMS (Oursinø, Palmettes, otc), par M.

COFALlIAIRES ou Po-

LYPES PROPREMENT DITS (Coraux, Gorgones, Eponges, etc.), par MM.MILNEEdwand et J. Haime, tomes 1 et 2 , arec 2 livraisons de planches. Fig noires, 19 fr., et fig. coloriées. $25 \mathrm{fr}$. INFUSOIRES (Animaleules microscopiques), par M. Dujardin, doyen do la Faculté des Sciences, à Rennes, 1 vol. a rec 2 livraisons de pì. (Ouv. termind.) Fig. noires. 12 f 30 Fig. coloriées, 18 f 50 BOTANIQUE (Introduction a l'étude de la), ou Traité élémentaire de cette science, contenant l'Organographie, la Physiologie, etc., par ALPE.DE CANDOLLE, pro. pesseur d'Histoire naturelle Genèvo (Ouvrage termine, autorisé par l'Unieersite pour les collèges roysux et communaux), \& vol. ot $8 \mathrm{pl}$.

VEGETAUX PRANEROGAMES (Organes sexuels apparents, Arbres, Arbrisseaux, Plantes d'agrément, otc.), par M. SpAca, aideaaturalists au Muséum 
d'Alistoire maturelle; 147. et 15 livr. do $\mathrm{pl}$., (ouvrage termind) fig. noire $156 \mathrm{fr}$. Eig. coloriées. $181 \mathrm{fr}$. CRYPTOGAMES, à Organe: sexuels peu apparents ou cachés, Mousses, sougères, Lichens, Champignons, Truffes, etc., par M. Brábisson, de Falaise. GLOLOGIF (Histoire, Formation et Disposition des Matériaux qui composent l'écoreo du Globe terrestre): par M. Fuot, membre do plusieurs Sociétés savantes. 2 rol. ensemble de plus de 1500 pages, avec un atlas de $24 \mathrm{pl}$. (Ouv.terminé.) $13 \mathrm{fr}$. MINERALOGIE (Pierres, Sels, Métaux, etc.) par M. Derafosse, membre de l'Institut, professeur au Muséum d'Fistoire nata. relle et à la Sorbonne.

Tome 1 er arec deux livr. de planches.

$12 \mathrm{fr} .50$

\section{CONDITIONS DE 2.A SOUSCRIPTIOE.}

Lov SUITES à BUFFON formeront cent volares in-8 environ, inprimés avec le plus grand soir ế ge bea papier; ce nombre paraît suifisant pour donner cet ensomble toute l'étendue convenable. Ainsi qu'il a ôte précédomment, chaque auteur s'occupant depuis longtempos Ie la partie qui lui est confiée, l'Editeur sera à même de par blier en pou de temps la totalité de traités dont so cempowera cette utile collection.

En décembre 1858, 65 rolumes sont an qente, avec 66 livraisons de planches.

Los personnes qui roudrent sonscrire pour tonte la Colİetion auront la liberté de prendre par portion justănà ce Fुn'elles soient su courant do tout ce qui paru.

\section{SOUE LES SOUSCRIPTEE}

Prix du texte, change volume (1) d'entiron 500 s p\$ges.

Prix 1 e change litraison d'environ 10 pl. noires. - coloriẻes. \& fr.

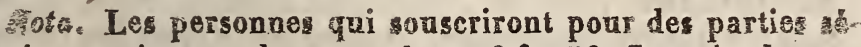
parées, paieront chaque volume $6 \mathrm{fr}$. 50 . Le prix des lumes papier vélin sera double du papier ordinairo.

(1) L'Éditeur ayant à payer poar astt eollection des hoboraire ans auteurs, le prix des volumes ns pert être comparé à celui des réimgrezions d'ourrages appartensnt a domaine public et exempts de

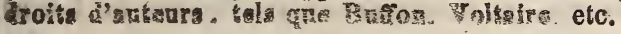




\section{ANCIENNE COLLEGTION}

DES

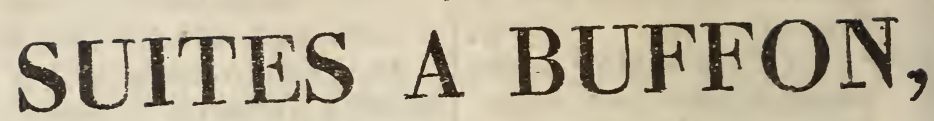

FORMAT IN-18;

Fermant ave les OZutros de cet Lestows

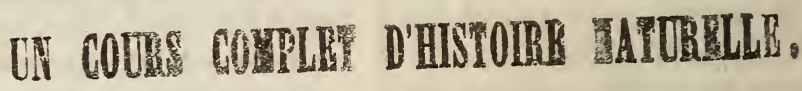

ลONTELKKF

- LES TROIS RËGNES DE LA NATURE;

Par Messieurs

BOSC, BRONGNART, SLOGT, CASTEL, GUERIK, DI LALAXCK, LATREILLE, BE BIRBEL, PATRIN, SONNINI SDE TIGRY;

LA glapsst

Cetśc Collection: primitivement publice par les soims de

- Déierville, et qui est devenue la propriété de M. Roret, ne peut être donnée par d'autres éditeurs, n'étant pas, comme les OEuvres de Buffon, dans le domaine public.

Les personnes qui auraient les suites de Lacépẻde, contenan seuleanent les Poissons el les Reptiles, auront la liberté de ne pas les prendre dans cette collection.

Fette Collection forme š volumes, ornẻs d'environ 600 pian ches, żessinées d'après nature par Desève, et précieusemen termines as burin. Elle se compose des ouvrages suivants

IISTOIRE ATURELLE DES INSERTES, compo. sée d'après Reaumur, Greofiroy, Degeer, Roesel, Linné Fabricius, et les meilleurs ouvrages qui ont paru sur celt partis, rédigé suirant les méthodes d'Olivier, de Latreille arec des notes, plusieur observations nouvelles et des figurez dessinée d'après nature: par .-M.-G. DI TIGrY e BRorgriarT, pour les généralités. Kdition ornéo do beau coup de figures, angmentée et wise au nirean des connais.

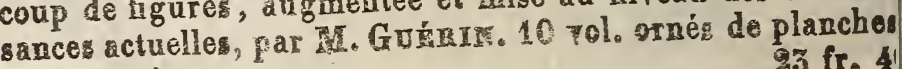
figures noires.

NATURELLY DES VGKTAUR classe par milles. of l'indication de l'usago qu'on peat fairo des planter dan 
Ios arts, lo commerce, l'agriculture, lo jardinage, la médecino, otc.; des figures dessinées d'après nature, et un GrFERA complet, relon le système de Linné, avec des renvois aux familles naturelles de Jussieu ; par J.-B. LAMARcK, membre de l'Institut, professeur au Muséum d'Histoire naturello, et par C.-F.-R. MIRbeL, membre de l'Académie des Sciences, professeur de botanique. Edition ornée de 120 planches représentant plus de 1600 sujetg. 15 volumes orné de planches, figures noires.

$30 \mathrm{fz} 90$

Le même ouvrage, figures coloriées. $\quad 46 \mathrm{fr} .50$

HISTOIRE NATURELLE DES COQUULLE, contenant leur description, leurs mœurs et lours usages, par 3. Bosc, membre de l'Institut. 5 vol. ornéz de planches. figures noires.

Le même ouvrage, figures coloriées.

10 is 65

$16 \mathrm{fr}, \mathrm{se}$

- NATURELLE DES VERS, contanant leur descrip-

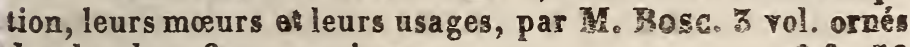
de planches, figures noires.

Lo même ouvrage, figures coloriées.

$5 \mathrm{H}: \mathrm{s} 0$

NATTREI DES CRUSTAC \& contenant

$10 \mathrm{fs} s 0$ description, leurs mours et leurs usages, par M, BoSC. 2 vol. ornés de planches, figures noire.

Lo même ouvrage, figures coloriées. 3 fite

竞 $\mathrm{fr} .75$

NATURELLE DES MINÉRAUZ, par M. B.

Patrif, membre de l'Institut. Ouvrage orné de 40 plan. ches, représentant un grand nombro de zujets dessinés d'a près nature. 5 volumes ornés de pląnches, figures noires

ํํำ

10 f: 50

16 10.

NATURELLE DES POISSONS, arec des figures dsssinées d'après nature, par BLoch. Ourrago classé par ordres, genre et espèces, d'après le système de Linné, avec los caractères génériques, par RÉ RichasD CASTRE. dition orné de 160 plancheø représentant 600 espèces dis poissons, 10 volumes.

Avec figures coloriées.

96 f.

4.

NATURELLE DES REPTILES, arec des igures dessinées d'après nature, par SoNrINI, homme de lettros et naturaliste, et LATREILLE, membre de l'Institut. Fition ornée de 54 planches, représentaut environ 150 espèces différontes do serpents, vipères, couleusres, lézaids, grenouilles, tortues, etc. 4 rol. arec planches, higures

Lo âmo vurrage, figures colorices.

9 ir. 85 $17 \mathrm{fr}$. 
Cett collection de 5̌z volumes a été annoncée en 108 demisolumes; on les enverra brochés de cette manière aux pors. sosne Gieni en feront la demande.

Tous les osvrages ci-dessus soní en venst.

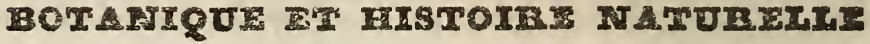

(Poir aussi la Collection «anusis, page 3.)

\section{ARPALES (NOUVELIES) DU MUSEUM D'PIS-} TUIRE NATURELLE, recueil de mémoires do MM. les professeur administrateur de cet établissement, ot autros zaturalistes célébres, sur les branche des sciences naturelles or chimiques qui y sont enseignées. Années 1832 \& 1835, sol. in-4. Prix : 30 fr. chaque volume.

APERCU SUP LES ANIMAUX UTILES ET NUIEIBIES de la Belgique, par Sélys-LoNGCHAMPs. 2 fr.

LES ARBRES ET ARBRISSEAUX de l'Europo of lours insecies, par MACQUART, in-8. $\quad 6 \mathrm{fr}$.

ARCHIVES DE LA FLORE DE FRANGE of DAE SEMAGNE, par ScruLTz. 1842. In-8.

Il paraîtra plusieurs feuille par an. Prix : 30 G par fiexille.

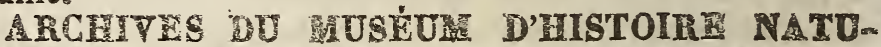

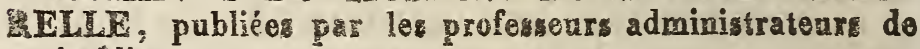
sot établissement.

Cet ourrage fail suito aux Amales, aux Mémoires aux Nouvelles Annales du Muséum.

Il paraît par volume in-4, sur papier grand-raisin, d'enFiron 60 feuilles d'impression, ef orné de 30 aे 40 planches gravéer par les meilleurs artistes, ot dont 15 \ 20 sont cole. ziées avec le plus grand soin.

Il onz paraî́t un volume par an, divisé on 4 livraisons.

Prix de chaque rolume $\left\{\begin{array}{l}\text { Papier ordinaire. } 40 \mathrm{fr} \text {. } \\ \text { Papier vélin. }\end{array}\right.$

BOTANIQUE (la), de J.-J. Rousseau, contenant toui ce fa'il a écrit sur cette äcience, augmentée de l'exposition de Ia séthode de Tournefori et de Linné, suivio d'un Dictionraire de botanique ot de motes historiques; par $\mathrm{K}$. DEVILZE. édit., 1 gros rol. in-12, ornè de 8 planchos. 4 fr.

Figures coloñées.

5 is.

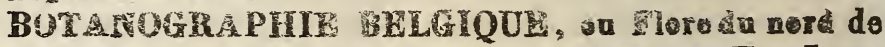
la France el de la Belgique proprement dite, par TH. LESTISOUDOIS. 2 rol. in-s.

$14 \mathrm{fr}$. 
BOTANOGRAPHIE ELEMENTAIRE, on Principe de Botsnique, d'Anatomio at de Phyaiclogio yégélale, par Tr. Listiboddors. in-8.

fr.

BOTANOGRAPHIE UNIVERSELLE, ou Tableau gẻnéral des Végétaux, par TH. LeSTIB जodors. 2 vol. in-8

$10 \mathrm{fr}$.

GALENDRIER DE FLORE, ou Liude de Fleurs d'aw près nature. 3 rol. in-8.

$10 \mathrm{fr}$.

CATALOGUE JES LEPIDOPTERES, ou Papillons do la Belgique. ptécédé da tableau des Libellutines de co pays, par M. DE SÉlys-LONGChALPS. In-8. 2 fr.

CA VERNES (des), de leur origins st te leur modo de formation, par Th. Virlert. In-8. 1 fr.

GEOIX DES PLUS BELLES FLEURS ET DES PLUS BEAUX FRUITS, par M. REDotTÉ. 1 joli vol. in-folio orné de 144 planches coloriées. 36 liqraisons de 4 planches à $6 \mathrm{fr}$. chaque livraison, soil pour l'ouvrage complet, qui est terminé, $216 \mathrm{fr}$.

Toutes les planches de l'ouvrage de M REDouté se vendent séparémenl à raison de 2 francs.

COLLECTIOI ICONOGRAPHIQUE ET HISTORIQUE DES CHENILLES, ou Description et figures de? chenilles d'Europe, arec l'histoire de leurs métamorphoses: et des applications à l'agriculture, par. MM. BoIsDUvAz; RAMBUR et Graslin.

Cotte collection se composera d'en piron 70 livraisons, 10 . mat grand in-8, et chaque lirraison comprendra trois plas ches solorizes et le texte correspondant.

Lo prix do chaqus livraison est de 3 fr. sur papier yolin, et franche de port $5 \mathrm{fr} .25 \mathrm{c}$. - 42 livraisone ont deja paru.

Les dessins des espèces qui habiteníles environs de Paris, comme aussi ceux des cherilles que l'on a envoyées vivanies a l'auteur, ont été zxécutés avec aulant de précision que de talewt. L'on continuera a dessiner toutes celles que l'on pourra se procurer en nature. Quant aux espèces propres à l'Allemagne, la Russie, la Hongrie, etc., elles seront peintes par les artistes les plus distingués de ces pays.

Le lexte est imprimé sans pagination; thaque espèce aura une page séparée, que l'on pourra classer comme on voudra. Au commencement de chaque page se trouvera le même nus méro qu'd la figure qui s'y rapportere, ef en titre le nom de la tribas, comme en tête de la planche.

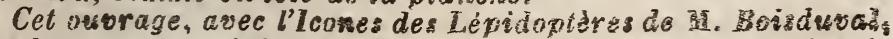
de beaucoup supérieurs d tout ce qui a paru jusqu'a présent formeront un supplément et une suite indispensable auss ouvrages de Hubner, de Godart, etc. Tout ce que nous powons 
dire en faveur de ces deux ouvrages remarquables peut se redwiró de cette expression employée par M. Dejean dans le cinquieme volume de son Species: M. Boisduval est de tous nos entomologistes celui qui connaî́t le miéux les lépidoptères

CONFGRENCES SUR LES APPLICATIONS DE 'ANTOMOLOGIE A L'AGRICULTURE, précédéo d'un discours, par M. Macedart. (Extrait des publications agricoles de la Société des aciences, de l'agriculture et der arts de Lille), br. in-8o.

$73 \mathrm{c}$.

CONSPECTUS SYSTEMATIS Ornithologiæ, in-po, pas 2y. Ie Prince Charles Bonaparte.

$2 \uparrow .50$

$\begin{array}{lll}\text { - Maslologiæ, } & \text { idem. } & \text { 2f.50 } \\ \text { - Herpetologiæ, } & \text { idem. } & \text { if.50 } \\ \text { - Icthyologiæ, } & \text { idem. } & \text { 2f.50 }\end{array}$

COUPE THEORIQUE DES DIVERS TERRAIYS. ROCHES ET MINERAUX qui entrent dans la composjtion du sol du Bassin de Paris, par MM. Covieg et Alexar. DEE ProNGMIART. Une feuille in-fol.

2 fr. 30

COURS D'ENTOMOLOGIE, ou de l'Histoire naturelle des crustacés, des arachnides, des myriapodes el des insactes, à l'usage des élèves de l'Ecole du Muséum d'Histoire asturelle, par M. LATreille, professeur, membre de l'Institut, etc., contenant !e discours d'ouverture du cours. - Tableau de l'histoire de l'entomologie. - Généralités de la classe des crustacés et de celle des arachnides, des myriapodes et des insectes. - Exposition méthodiquo des ordres: les familles, et des zyenres des troiz premières classes. gros rol. in-8, etun Atlas compozé de 24 planches. $15 \mathrm{f}$.

COURS D'HISTOIRE NATURELLE conforme 2u ตอนveau programme de l'Université, par M. FothNEI. 120 par= tie - Digne animal. In-8.

6 fr.

DESCRIPTION DES FOSSILES DES TERRAINS MIOCENES DE L'ITALIE SEPTENTRIONALE, p

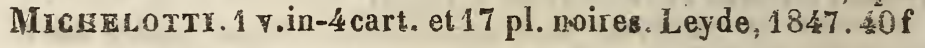

DESCRIPTION ET FIGURES DES, PLANTES AOUVELLES et rares du jardin botanique de Leydds, etc.

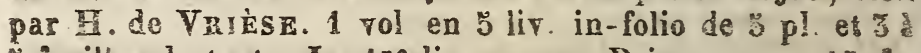
5 íeuilios de texte. La 1 ro liv. a paru. Prix

$15 \mathrm{fH}$.

DESCEIPTION GEOLOGIQUE DE LA PARTIE 
MERIDIONALE DE LA CHAINE DES YOSGES, par M. BozeT, capítaine au corps royal d'état-major. In-8 orné de planches et d'ùne jolie carte.

$10 \mathrm{fr}$.

- DESCRIPTION GÉOLOGIQUE DES ENVIRONS DE PARIS, pay MM. G. Cufier et A. Bronghiart. In-4, figures.

$40 \mathrm{fr}$.

DESCRIPTION DES MOLLUSQUES FLUVIATILES ET TERRESTRES DE LA FRANCE, ot plus paTticulièrement du département de l'Isère, ouvragge orué dè planches représentant plus do 140 espèces, par M. ALIN GrAS. In-8.

$3 \mathrm{ft}$.

-OURSINS FOSSILES (Des), ou Notions sur l'Organisation etla Glossologie da cette classe,p.ALBIN Gras. In-8. $6 \mathrm{fr}$.

DICTIONNAIRE DE BOTANIQUE MEDICALE ET PHARMACEUTIQUE, contenant les principales propriétés des minéraux, des végétaux et des animaux, avec les préparations de pharmacie, internes et externes, les plus usitees en médecine et en chirurgie, etc., par ane Société de médecins, de pharmaciens et de naturalistes. Ourrage utile à toutes les classes de la société, orné de 17 grandes planches représentant 278 figures de plantes gravées avec le plus grand soin, 30 édition, revue, corrigée et augmentée de beaucoup de préparations pharmaceutiques et de recettes

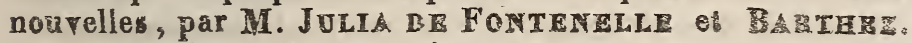
2 gros vol. in-8, figures noires. 18 f Le mếme, figures coloriées d'après naturt. $2 \mathrm{f}^{\mathrm{z}} \mathrm{s}$. Cet ouvrage esi spécialement destiné aux personnez qui sans s'occuper de la médecine, aiment à secourir les malheureus.

* DICTIONNAIRE (nouveau) D'HISTOIRE NATURELLE appliquée aux arts, à l'agriculture, à l'économie rarale ot domestique, à la médecine, etc., par une Société de naturalistes et d'agriculteurs, $36 \mathrm{vol}$. in-8, fig. noires. $120 \mathrm{fr}$.

Idem, figures coloriées.

* DICTIONNAIRE RAISONNE ET UNIVERSEL D'HISTOIRE NATURELLE, contenant l'histoire des an'maux, des végétaux et des minéraux, par VALMONT DH Bo MARE. 15 rolumes in-8.

35 fr.

DILUVIUM $(d u)$. Recherches sur les dépôts suxque's on doit donner co nom et sur la cause qui les a produits, par M. Melleville; in-8.

2 fr. 50 .

DIPTERES DU NORD DE LA FRANCE. Par M.J. MaceUart. 2 volumes in-8.

DIPTERES EXOTIQUES

NOUYRAUX OU PEU 
CONNUS, par M. J. Macedart, membre de plusieurs sociétés avantes; 1 . 1 et 2,5 livraisons in -8 prix, figures noires.

$35 \mathrm{fr}$.

3. nême ouvrage, fig. colorièen $60 \mathrm{fr}$

Les Suppléments 1 à 4 (1846-5̃1), chaque : fig.noires, $7 \mathrm{fr}$., fig. col.

$12 \mathrm{fr}$.

5 (1855), fig. noires, 4 fr ; fig. col. 6 fr.

DISCOURS SUR L'AVENIR PHYSIQUE DE LA FERRE, par Marcil or Serras, professeur de minéralegie et de géologie à la Faculté des Sciences de Montpellier, in-8; pris

2 fr. 50

ELIMENES DES SCIENCES NATURELLES, par A.-M. CONStant-DumériL. $5^{*}$ édition, 1846, 2 vol. in 19, fig.

ENUMCRATION DES ENTOMOLOGISTES VIVAXTS, suivie de notes sur les collections entomologistos des musées d'Europe, etc., avec une table des résidences des ertomologistes. Par Silbermain, in-8.

$3 \mathrm{fr}$,

LSSA I MONOGRAPHIQUE ur lo Campagnols dta environs de Liège, par M. DE ŚlLYS-LONGGHAMPS, in-8, figures.

$3 \mathrm{fr}$.

ESSAI SUR L'HISTOIRE NATURELLE DU BRABANT, par feu M (Mammifères.) $2 \mathrm{fr} .5 n$

(Analyse et Extraits par M. DE SÉLY LONGCHA MPS.)

ESSAI SUI L'HISTOIRE NATURELLE DES SER PENTS de la Suisse, par J. F. WYDER. in-8, fig. 2 fr. 36 ESSAIS DE ZOOLOGIE GÉNERALE, ou Memoires of notices ur la Zoologie générale, l'anthropologie et l'his toire de la science, par M. ISIDORE GEOFFROY SEINT $-\mathbb{Z I}$.

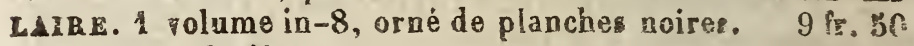

Figures soloriées. 12 iा 50

ETUDE DE MICROMAMMALOGIR, revae des sorex, ot arvicola d'Europe, suivies d'un index métho. dique dés mammifères européens, par M. EDOH. DE SÉEY LONGCBABRS. 1 volume in-8.

3 is

ETUDES PROGRESSIVES DUN NATURALISTE. pendant les années 1834 et 1835 , par M. R. GrofrroY Saint-Hillatre. Paris, 1835, in-4. $13 \mathrm{fr}$. ETUDES SUR L'ANATOMIE et lá Physiologie des Vógétaux, par Tue Lestiboudois. in-8, fig. Â. EUROPEORUM MICROLEPIDOPTERORUM IndeX 
methodicus, sive Spirales, Tortrices, Tinea et Alucitæ Linnæ1. Auct. A. Góñée. Pars prima, in-8.

3 fr. 75

FACULTÉS INTÉRIEURES DES ANIMAUX INVERTÉBRÉS, par M. MACQUART, 1 vol. iu-80. $5 \mathrm{fr}$.

FAUNA JAFONICA, sive Descriptio animalium quaia itinero per Japoniam jussu el auspiciis superiorum, qui summum in India latava imperium tenent, suscepto anai s 1823-1830, collegit, notis, observationibus et adumbra= tionibus illustravit PH. Fr. DE Siebold. Prix de chaque litTrnikon : $26 \mathrm{fr}$. en noir; celles en couleur $32 \mathrm{fr}$.

C.et ouvrage, auquel participent pour sa rédaction ang. Temminok, Schlegel et Dehaan, se continue avec activité. 41 livraisons sont en vente; savoir: Mammalogie, 3 liv.; Reptiles, 3 lit. Crustacés, 7 liv.; Poissons, 16 liv.; Oiseaux, 12 livr.

FAUNE DE L'OCEANIE, par le docteur BoIsDUVAR. Un gros vol. in-S, imprimé sur grand papier vélin. $10 \mathrm{fr}$.

FAUNE ENTOMOLOGIQUE DE MADAGASCAR, BOURBON ET MAURICE. - Lépidoptères, par le do teur Borsduval; avec de notes sur les métamorphoses, par M. Sganzin.

Huit livraisons, renfermant chacure \& pl. coloriées, arec le texte correspondant, sur papier vélin. 32 fr.

FAUNE (sur la) DE LA BELGIQUE, par de SÉlysLONGCBAMPS, br. in-8.

$1 \mathrm{fr}$.

FILLE BICORPS de Prunay (sous Abli), connue dans la science sous le nom de Ischiopage de Prunay, par M. Groffroy Saint-Hilaike. In-4. Figures. 3 fr.

FIORA JAPONICA, sive Plantæ quas in imperio Japonice collegit, descripsit, ex parte in ipsis locis pigendas curavit, D. Ph.-Fr. de Siebold. Prix de chaque livraison 16 fr. coloriée, el 8 fr. noire. Il ex paraît 35 livraisons.

FLORA JAVE nec non insularum adjacentium, auctore

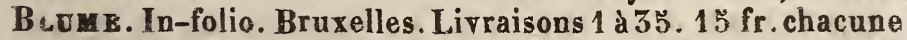

FLORE DU CENTRE DE LA FRANCE et du bassin de la Loire, par M. A. Borgav, directeux du Jardin des Plantes d'Angers, etc. $3^{0}$ édition. 2 rol. in-8; prix : 15 fr.

FLORE DE L'ARRONDISSEMENT D'HAZEBROUCK, par VANDAMME, 2 vol. in-8. $4 \mathrm{fr}$. 50

FLORE DES JARDINS ET DES GRANDES CULTURES, etc., par SERINGE. 3 vol. in-80. 27 f.

FRAGMENTS BIOGRAPHIQUES, précédẻs d'études sur la vie, les ouvrages el les doctrines de Buffon, par M. Geoffhop Saint-Hilaire. In-8. 
GENERA ET INDEX METHODICUS Europæorum Lopidopterorum, pars prima sistens Papiliones sphingos, Bombyces noctuas, auctore BoISDUVAL. 1 vol. in-8. 5 fs.

GERBARII TIMORENSIS DESCRIPTIO, cum tabulis 6 æneis; auctore J. DecaISNe. 1 vol. in-4. $13 \mathrm{fr}$.

HERBIER GÉNÉRAL DES PLANTES DE FRANCE ET D'ALLEMAGNE, par M. ScholTz. In-folio, livraison: 1 à 4 .

$20 \mathrm{fr}$. chacune.

* GISTOIRE ABREGÉ DES INSEGTES, Par M. GEOFFROY. 2 vol. in-4, figures.

$25 \mathrm{fr}$.

HISTOIRE DES MOEURS ET DE L'INSTINCT DES ANIMAUX; distributions naturelies de toutes leurs classes: par J. J. VIREX. 2 vol. in-8.

$12 \mathrm{fr}$

HISTOIRE DES PROGRES DES SCIFNCES NATURELLES, depuis 1789 jusqu'en 1831 , par M. lo baron G. Cuvier. 5 rol. in-8. 22 fr. 30.

Le toms 5 séparément. ifr.

Le Conseil royal de l'Université a décidé quo cet ouvrage serait placédans les bibliothèques des colleges et donné en prix ause élèves.

HISTOIRE NATURELLE DES LÉPIDOPTÉRES, RHOPALOCERES, ou Papillons diurnes des départements des Haut et Bas-Rhin, de la Moselle, de la Meurthe el des Vosges, publiéa par L. P. Gantener. 13 livraisons in-8, fig. col.

$26 \mathrm{fr}$.

HISTOIRE NATURELLE ET MYTHOLOGIQUE DE L'IBIS, par J.-C. SAVIGNY. in-8, a vec $6 \mathrm{pl}$. $4 \mathrm{fr}$.

* HISTOIRE NATURELLE GÉNÉRALE ET PARTICULIERE, par M. le comte de BUFFON; nouvelle édition accompagnée de notes, etc.; rédigée par M. SonNINI. Paris, Dufart, 127 vol. in-8.

$300 \mathrm{fr}$.

HISTOIRE NATURELLE, ou éléments de la Faune française, par MM. Braguier et Madritte. In-12, rahiers 1 à 5 , à 2 francs chaque.

$10 \mathrm{fr}$.

ICONES HISTORIQUES DES LÉPIDOPTĖRES NOUVEAUX OU PEU CONNUS, collection, avec figures coloriées, des papillons d'Europe nou vellement découverts; ourrage formant le complément de tous les auteurs iconographes ; par le docteur BoISDUVAL.

Cet ouvrage se composera d'environ 50 livraisons grand in -8 , comprenant chacune deux planches coloriées et le tex te correspondant; prix, 3 francs la livsaison sur papier vélin, et franche de port,

3 fr. 25. 
Comme il est probable que l'on découvrira encore des ospsces nouvelles dans te contrées de l'Europe qui n'ont pas dis bien explorées, l'on aura soin de plublier, chaque année, une ou deux livraisons pour tenir les souscripleurs au courant des nouvelles découvertes. Ce sera en même temps un moyen trèsa tanlageux et très-prompt pour MM. les entomologistes, qui auront trouvé un lépidoplère nouveau, de pouvoir les publier les premiers. C'est-ä-dire que, si, après avoir subi un examen nécessaire, leur espèce est ґéellement nouvelle, leur description sera impriméetextuellement; ils pourronl même en faire tirer quelques exemplaires à part. - 42 livraisons ont déjà parn.

ICONOGRAPHIA DELLA TAUNA ITALICA; A Ánolo-LucIano BoNAPARTE, 30 livraisons in-folio 21 fr. 60 chaque.

ICONOGRAPHIE ET HISTOIRE DES LEPIDOPTERES ET DES CHENILLES DE L'AMEHIQUE SEPTENTKIONALE, par le docleur BoISDUVAl, el par le major Johr LeconTE, de New-York.

Les livraisons 1 à 26 sons en vente, el les suivantes paraîtront à des intervalles très-rapprochés.

L'ouvrage comprendra environ 50 livraisons. Chaque livrai3 contient 3 planches coloriées, et le texte correspondant. Prix pour les souscripteurs, $3 \mathrm{fr}$. la livraison.

ICONOGRAPHIE ET HISTOIRE NATURELEE JES COLÉOPTERES D'EUROPE, famille des Carabiques, par M. le comte DEJEAin et M. le docteur BoISD tvas. 46 livraisons gr. in-8, fig. col. A 6 fr. la liv. 276 fr.

ILLUSTRATIONES PLANTARUM ORIENTALIUM, ou Choix de Plantes nouvelles ou peu connues de l'Asie occidentalo, par M. le comte JAURER et M. SPACH. Cet ou = zrage forme s vol. arand in-4, composés chacun de 100 plasche et d'environ 30 feuilles de texte; il a paru par ivraisons 10 planches. Le prix de chacune eat do $15 \mathrm{fr}$. L'ourrage complet (50 livraisons à $15 \mathrm{fr}$.)

$750 \mathrm{fr}$.

INSECTA CAFFRARIA, annis 1858-45, a J.V.VAELBerg, collecta descripsit Ganolos H. BoHewan. Pars 1. Fasc. 1. Coleotrina (Carabici, Iydrocanthari, Gyrivi el Staphylinii). 1 vol. in-80:

$8 \mathrm{fr}$.

Fasc. 2. Coleoptera (Buprestides Clatérides, Cébrionites, Rhipicériỏes, Cyphonidez, Lycides, Lampyrides, etc. In. 80

$10 \mathrm{fr}$.

Pars 2. Coleoptera (Scarabæides), in-80

$10 \mathrm{fr}$.

INSECI A SUECICA, descript a Leonardo GYLLEHAL. Scaris, 1808 à 1827. 4 rol, in-8.

$48 \mathrm{fr}$. 
INTRODUCTION A L'ÉTUDE DE LA BOTANIQUE, par Pailibert. 3 vol. in-80; fig. col.

$18 \mathrm{fr}$.

MÉMOIRES DE L'ACADÉMIE DES SCIENCES WT LETTRES DE MONTPELLIER. - Mémoire do la section des sciences, 1847-1848. 2 forts vol. in $-4^{\circ}$ aree Eg. Chaque.

$6 \mathrm{fr}$.

MÉMOIRE S SUR LA FAMILE DES COMBRETA . GEE, par M. DB GANDOLLE. In-40; fig. ifr.

MÉMOIRE SUR LES TERMITES observé à Rochofort et dans divers autre lieux du département de la Cha. rente-Inférieure, par M. BовE-Moread. In-80. 3 fr

MÉMOIRES DE LA SOCIÉTÉ DE PHYSIQUE DE GENEVE, in-40. - Divers Mémoires séparés sur les Selaginées, les Lythraires, les Dypsacées, le Mont-Somma, ote.

- DE LA SOCIÉTÉ D'HISTOIRE NATURELLE DE PARIS. 5 vol. in $-4^{0}$ arec planches. Prix : $20 \mathrm{fr}$. chaque rolume. Prix total. $100 \mathrm{fr}$.

- DE LA SOCIETE ROYALE DES SCIENCES DE LIĖGE. Tome 1 1843, in-80.

- Tome 2, 1845.

$8 \mathrm{fr}$.

- Tome 3, 1845 (contenant la Monog. des Coléoptères abpentamères-phytophages., par LACORDAIRR, t. 1). 12 fr.

- Tome 4, 1847-49, (contenant la monographie des Proảuctus, par M. de KonincK). 2 vol. in 8 et un atlas. La $1^{\text {ro }}$ partie, 1 vol. et 1 atl. $10 \mathrm{fr}$. La $2^{\mathrm{e}}$ partie, 1 vol. $5 \mathrm{fr}$.

- Tome 5, 1848. Monog, des Coléoptères subpentamèrezphytophages, par M. Lacordaire, tome 2.

- Tome 6, 1849. Monog. des Odonales.1 rol. 10 fr.

- Tome 7, 185̃1. Exposé élémentaire de la Théorio des Iotégrales définies, par MeYer. 1 vol. in-80.

$10 \mathrm{fr}$.

- Tome 8, 1853. renfermant le catalogue des larves des Coléoptères connues jusqu'à ce jour, a vec la description de plusieurs espèces nouvelles, par MAM. Chapuis et de CaNDËZE. $12 \mathrm{fr}$.

- Tore 9,1854, contenant la Monographie des Caloptérygines, par M. DE SÉlys-LoNgGHAMPS. 1 roi. in-8. 12 fr.

- Tome 10, 1856. Cours élémentaire sur la Fabrication des bouches à feu en fonte et en bronze, par Coquilhat. $1^{\text {re }}$ partie. $\mathrm{n}-8$.

$12 \mathrm{fr}$.

- Tome 11, 1858. Fabrication des bouches à feu, par Coloillat. $2^{\ominus}$ parlie. - Calcul des rarialions, par $\mathbf{A}$. 
MeYBr. - Monographie des Gomphines, par De SÉlysLONGCHAMPS. 1 vol. in- ${ }^{\circ}$.

$18 \mathrm{fr}$.

- Tome 12, 1857. Monographie des Élatérides, par E. de Candèze. in -8 , prix

8 fr. 50

- Tome 15, 1858 Fabricalion des bouches à feu, par Copoilhat. $3^{\text {e }}$ partie. - Etudes sur un mémoire de Jacobi, relatif aux inlégrales définies, par N. - C. SchмIтT. - Notice géologique, par J. van BinkHorst. 1 vol. in $-8^{\circ}$. $12 \mathrm{fr}$.

* MÉMOLRES pour servir à l'Histoire des Inzectes, par DB RÉAOMUR. 6 vol. in-40.

$50 \mathrm{fr}$.

MÉMOIRES SUR LES ANIMAUX SANS VERTE: BRES, par J.-C. SAVIGNY. Paris, 1816, $1^{\text {re }}$ partie, promier fascicule, a vec $12 \mathrm{pl}$.

$6 \mathrm{fr}$.

- 20 partie, premier fascicule, avec 24 pl. col. $28 \mathrm{fr}$. MÉMOIRES SUR LES METTAMORPHOSES DES COLEOPTĖRES, par DE HAAN. In-40; fig.

$10 \mathrm{fr}$.

MONITEUR ( $\mathrm{l} \theta$ ) DES INDES orientales et occidentales, Recueil de Mémoires et de Notices scientifiques ot industrielles, etc.; publié par F. DE SIÉBOLD et P. MELTILL DE CARNBÉ E. 1846, no\$ 1, 2, כ, un cahier in-4.

MONOGRAPHIE DES EROTYLIENS, famille de l'or. dro des Coléoptères, par M. Th. LACordaIrk. In-8. 9 f

- DES LIBELLULIDÉES D'EUROPE, par Edm. DE SÉLys-Longchamps. 1 vol. gr. in-8, avec quatre planches roprésentant 44 figures. Prix :

$5 \mathrm{fr}$.

MONOGRAPHIA CASSIDIDARUM auctore CAROlo H. Boheman. Tomi I, II, III, cum tab. VII. Holmia $(1850 \cdot 55) 3$ vol, in 8 , chacun $14 \mathrm{fr}$.

MONOGRAPHIA TRYPHONIDUM SUECIE, auctore AUg. EMIL. Holm Gren, in-40

$13 \mathrm{fr}$.

NOTICE SUR LES DIFFÉRENGES SEXUELLF des Diptères du genre Dolichopus, tirées des nervures des ailes; par M. MACQUART. 1844 , in-8.

1 is.

INOTICE SUR h'HISTOIRE, les Mour et l'Organissetion de la Girafe, par M. JoLY. In-8.

$1 \mathrm{fr}$.

NOTICES SUR LES LIBELLULIDEES, oxtraite des Bulletins de l'Académie de Bruxelles, par Edm. DE Stúlys= LONGCHAMPS. In-8, fig.

2 fr:

OBSERVATIONS BOTANIQUES, par-B.C. DUEOR: TIER. In -8.

$4 \mathrm{fr}$

- OISEAUX AMERICAINS (Sur les) admis dans la Fauno européenne, par M. SÉLYS-LoNGGHAMPS, 1 volume in-80.

1 fr. 23 
OBSERVATIONS SUR LES PHÉNOMÉNES PÉBIODIQUES DU REGNE ANIMAL, et particulièrement - ar les migrations des oiseaux en Belgique de 1841 a 1846 , résumées par E. DE SélyS-LoNGGHAMPs. Brochure ir-40, prix :

3 fr. 30

ORNITHOLOGIE EUROPÉENNE ou Cátalogue anaIytique et raisonné des oiseaux observés en Europe, par M. Degland. 2 vol. in $-8^{0}$.

$18 \mathrm{fr}$.

FAPILLONS D'EUROPE peints d'après nature, par ErNst. 8 tomes en 4 vol. in-4, a vec $342 \mathrm{pl}$. col. $200 \mathrm{fr}$.

"PAPILLONS EXOTIQUES DES TRUIS PARTIES U MONDE, l'Asie, l'A frique et l'A mérique, par F. CraMERS. 4 vol.in-4, rel, avec 400 planches coloriées. $400 \mathrm{fr}$.

PLANTES (les), Poème, par R. R. Castel; nouvelle édition, ornée de š figures en taille douce. In-18, $3 \mathrm{fr}$.

PLANTES KARES DU JARDIN DE GENEVE, pas A. P. DE CANDolle; livraisons 1 à 4 , in-4, fig. col., à Es fr. la livraison. Prix total:

$60 \mathrm{fr}$.

PLANTES HERBACÉSS D'EUROPE ET LEURS INSECTES, par M. MACQUART, in- $\delta^{0} .1^{\text {re }}$ parlie, 3 fr. 50 ; $2^{\circ}$ partie, $5 \mathrm{fr}$; $5^{e}$ partie,

$4 \mathrm{fr}$.

REGA PITULATION DES HYBRIDES OBSERVÉS DANS LA FAMILLE DES ANATIDEES, par E. DE SÉlYS-LONGCHA y $P$, brochure in-80.

$1 \mathrm{fr} .25$

Addition a la Récapitulation, br. in-8. $1 \mathrm{fr}^{\circ}$

RÉGNE ANIMAL, d’après M. DE BLAINviLLz, disposé on séries, en procédant de l'homme jusqu'à l'épongo, et divisé en troir sous-règnes; tableau supérieurement grapé. Prix:

3 fr. 50

Collé gur toile, arec gorge et roulean.

8 fo.

REVUE ENTOMOLOGIQUE, publiée par G. SILEE = MiNN. Strasbourg, 1833 a 1837 ; 5 vol. in- 8.36 fre par an. ( 2 rol.)

*RUMPHIUS (G. Evr.); Cabinet des raretés de l'île d'Am boine (en hollandais). Amsterdam, 1705 ; in-folio, fig. 50 f.

* RUMPHII (G. Ev.) Herbarium Amboinense, Belgice ot Lat., curs et studio I. BurranNI. Amstelod, $1750 ; 7 \mathrm{rol}$. in-folio

$200 \mathrm{fr}$.

RUMPAIs, sive Commentationes botanicæ imprimis de plantis Inuias Orientalis, tum penitus incognitis, tum quæ in libris Rheedii, Rumphii, Roxburghii, Gallichii, aliorum recen 
sentar, auctore C.-L. BLJa R, cognomine RU mpHIO. Lo priz dechaque livraison est fixé, pour les souscripteurs, $15 \mathrm{fr}$. L'ouvrage complet, 40 livraisons,

$600 \mathrm{fr}$.

SERRES CHAUDES DU MUSÉUM D'HISTOIRE NATURELLE, ou Notice sur les Constructions du Jardin des Plantes, par M. RoHadLT, architecte. in-folio. $30 \mathrm{fr}$.

SINGULORUM GENERUM CURCULIONIDUM unare alteramve speciem, additis Iconibus a David LABRARE, illustravit L. I - SPECIES GENERAL DES COLÉOPTERES, अं M. DRJEAN, arec les Hydrocanthares de M. AUBk. T rol. in $-8^{\circ}$.

$100 \mathrm{fr}$.

L'on vend séparément le tome $\nabla$ en deux parties (co rolume a étẻ détruit dans un incendie). $\quad 35 \mathrm{fr}$.

SYNONYMIA INSECTORUM.-GENE⿱RA YT SPE. CIES CURCULIONIDUM (ourrage comprenant la synonymio et la description de tous les Curculionides connus), par

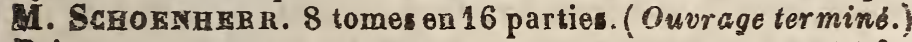
Prix :

$144 \mathrm{fr}$.

GURCULIONIDUM DISPOSITIO metbodica cum genesem characteribus, descriptionibus atque observationibus variis, seu Prodromus ad Synonymiæ insectorum partem IV, anetore C.-J. SCHOENHERR. 1 vol. in-8. Lipsia, 1826

ติ

L'éditeur vient de recevoir do Suède et de mettre en vente le petit nombre d'exemplaires restant de la Synonymia insectorum du même auleur. Chaque volume qui compose ce dernier ouorage est accompagné de planches coloriées, dans lesquelles l'auteur a fait représenter des espèces nouvelles.

SYNONYMIA INSECTORUM. Oder Versuch, te. SCHORNHERR. Skara et Upsaliæ, 1817. 4 vol. in-8. $50 \mathrm{fr}$.

SYNOPSIS DE LA FLORE DU JURA septentrional et du Sundgau, par Frighe-Joset et Montandon. 1 vol. in 12.

$5 \mathrm{fr}$.

- SPECTACLE (le) DE LA NATURE, ou Eutretien. sur l'Histoire naturelle, suivi de l'Histoire du Ciel, pai PLECHE. 11 vol.in-12. $20 \%$.

STATISTIQUE GÉOLOGIQUE ET MINERALOGTQUE du Département de l'Aube, par A. LeTMrRis. Troyes, 1846,1 vol. in -8 et Atlas in-4. Prix

$15 \mathrm{fr}$. 
TABLEAU DE LA DISTRIBUTION MÉTHODIQUF DES ESPĖCES MINÉRALES, suivie dans lo cours do minéralogio fait au Muséum d'Histoire naturelie en 1833, par M. Alexandre Brongriart, professeur. Brochure in-8.

$2 \mathrm{fr}$.

TABLEAU DU RÈGNE VÉGÉTAL, d'après la méthodo de A.-L. De Jussiev, modifiée par M. A. Richard, comprenant toutes les families naturelles; par R. Ch. D'ORBIGEY. $2^{6}$ édition; 1 feuille et quart in-plano.

idem, coloriés.

$2 \mathrm{fr}$.

$3 \mathrm{fr}$.

TAILLE DU POIRIER ET DU POMMIER en fusean. par Choppir. 1 vol. in-8 ${ }^{\circ}$, fig. $2^{\text {me éd. }}$

THÉORIE LEMENTAIRE DE LA BOTANIQUE, ou Exposition des Principes de la Classification naturello é de l'Art de décrire el d'étudier les végétaux, pas M. DE Camboler. $3^{\circ}$ édition; 1 vol. in-8.

$8 \mathrm{fr}$,

+ TRAITÉ ANATOMIQUE de la Chenille qui ronge lo bois de saule, par LionNeT. In-4. figures.

36 ir.

- ELÉMENTAIRE DE MINÉRALOGIE, par F.-8. Brodant, de l'Académie royale des Sciences, nouvelle éditio nconsidérablement augmentée. $2 \mathrm{vol}$. in-8, accompagnés de 24 planches.

21 fr.

ZEITSCHRITT FUR DIE ENTOMOLOGIE herausgegeben von Ernst Friedrich Gramar. Leipzig, 1839 i 1844. 5 rol. in-8.

$52 \mathrm{fr}^{\circ}$

ZOOLOGIE CLASSIQUE, on Histoire naturelle du hègne animal, par M. F.A. Poochet, professeur de zoologie an Muséum d'Histoire naturelle de Rouen, etc.: sesonde édition, considérablement augmentée. 2 vol. in-8, zontenant ensemble plus de 1,300 pages, et accompagnés d'un Atlas de 44 planches et de 5 grands tableaux gravés sur acier. Prix des 2 rol.

Prix de l'A tlas, figures noires. $16 \mathrm{fr}$. figures coloriées $10 \mathrm{fr}$. 30 f..

Mota. Le Conseil de l'Oniversité a décide que cet ouvrago serait placé dans les biblintheques des colleges. 


\section{AGRICULTURE,}

\section{ÉCONOMIE RURALE ET JARDINAGE.}

( Posir aussi la Collection do Nanuels, page 3.)

ABREGE DE LART VETERINAIRE, NU Descrit tion raisonnée des Maladies du Cheval et de leur Traitemens, saivi do l'anatomie et de la physiologie ảu pied et des principes do ferrure, avec des observations sur le régime ot l'exercice du cheval, etc., par WuITE; traduit de l'anglai ot annoté gar M. Y. DELAGEETTE, vétérinairo. $2^{\text {Médition }}$ in-12.

$3 \mathrm{fr} .50$

AGRICULTEUR-PRATICIEN (I'), REVUR B'AGR -

CULTURE, DE JARDINAGE, IT D'ECNOHE DE-

RALR ET DOSESTIQUR, sous la direction de MM. BOSGY

MALEPEYRE, f. Heozé, etc., in-8, grand furmat, ren.

fermant des gravures sur bois intercalées dan li texte.

Il a paru 14 années de ce Recueil, qui a commencé le 1 el cctobre 1839. Prix de chaque année, 5 fr. au lieu de $6 \mathrm{fr}$.

AGKICULTURE FRANCAISE, par MM. Ies Inspeeteurs do l'agriculture, publiéo d'après les ordres de M. le Winistre de l'Agriculture et dv Commerce, contenant la lescription géographique, le sol, le climat, lo populatios los exploitations rurales; instruments aratoires, engrais, as solements, otc., de chaque département. 6 vol., accompagnés thacun d'une belle carte, sont en vente, savoir :

2épartemeut de l'Isère. 1 vol. in-8.

5 fr.

- du Nord. In-8.

-- Les Hautes-Pyrénées. In-8 5

le la Haute-Garonne. In-8.

des Côtes-du-Nord. Tn-8. 5

$-d u$ Tarn.

5
5
5
5
5

AGRICULTURE DES ANCIENS, paY Hexser: tra duit de l'anglais. 2 7ol. in -8.

$10 \mathrm{fr}$

PRATIQUE des différentes parties de "ugleterro par MAESCHAL. S vol. in-8 et Atlas. $20 \mathrm{fr}$.

ALIMENTAIRES (des Conserves), nouveau procédé, par M. Willadiez. In-12. 2 fr. 23

AMATEUR DES FRUITS (1'), on l'Art de les choisir. de les conserver, de les smployer, principalement pour faire 
les compotes, gelées, marmelades, cunfitures, etc., par M. L. Durors. in-12.

$2 \mathrm{fr} 30$

AMÉLIORATION (De l') DE LA SOLOGNE, par

- M. R. Pareto. In 8.

2 fr. 50

AMPELOGRAPHIE RHÉNANE, par STOLTz, 1 rol. gr. in $\rightarrow$, fig. noires.

Le meme ouvrage, fig. col.

$17 \mathrm{fr}$.

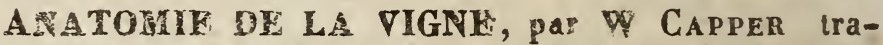
duit de l'anglais par $\nabla$. DE Molrox. In-8.

3.

ANHMAUX (les) C:LLEBRES, necdote bizteriques sar les traits d'intelligence, d'adresse, de courage, de bonté, d'attachement, de reconnaissance, etc., des animaux de toute espèce, orués de grav., par $\mathbf{A}$. ANtorn. 2 v. in-12 $2^{3}$ édition. 5 fr.

ANNALES AGRICOLES DE ROVILLE, ou Mélanges d'A griculture, d'Economie rurale et de Législation agricole par M. C.-J.-A. Mategieu DE Dombasle. 9 rol. in-8, figures.

$61 \mathrm{rr} .36$

Les volumes se vendent séparément, saroir :

Les tomes 1, 2, 3, 4, chacun

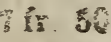

I $5,6,8$ et supplément, chacur

6 i.

ANNUAIRE DU BON JARDINIE GRONOME, renfermant la description et la culture de toutes les plantes utiles ou d'agrément qui ont parv pors la première fois.

Les années $1826,27,28$, chucun

Les années 1829 et 1830 , idem

Les années 1831 z 1842 , idem

1 1 ir. 30

$3 \mathrm{fr}$.

3 fr. 30

APPLICATION (DEl') DE LA NOUVELLI LOI SUR LA POEICE DE LA CHASSE, en ce qui regarde l'agricalture et la reproduction des animaux; par 2.-L. GADEBLAD. In- 8.

3 fr. 50

APPLICATION (De l') DE LA VAPEUR A L'AGRI. CULTURE, deson Influence sur les Mours, sur la Prospórifé des Nation et l'Amélioration du Sol par GIRARD。 Grand in-8.

ART (1') DE COMPOSER ZT DECOEER LES JALIVIS, par M. PBolTako; ourrage entièrement neuf, orné de 140 planches gravéo zur aciez. Priz de l'ourrage complet, texte et planches.

$151 \mathrm{f}$.

Celte publication n's rien de commus avec les autres nos. vrages du même genre, portant mêrne le nom de l'auteur. Le

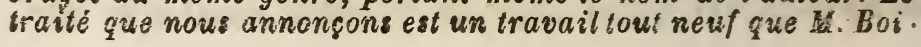


tard vient de terminer après des travaux immenses; il est très-complet et à très-bas prix, quoiqu'il soit orné de $14 \dot{s}$ plan. ches gravées sur acier. L'auteur é l'éditeur ont donc renau un grand service aux amateurs de jardins en les mettant à même de tirer de leurs mropriélés le meilleur parti possible.

ART (l') DE CREER LES JARDiNS, contenant les préceptes généraux de cet art, leur application développé pardes rues perspectives, coupe é élévations, pazdes exemples choisi dan les jardins les plus célèbres de Franco et d'Angleterre; et lo tracé pratique de toutes espèces do jar-

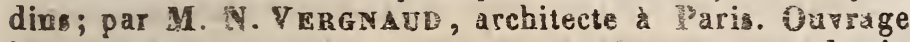
imprimé sur format in-fol., et orné de lithographios dessinées par nos meilleurs artistes.

$\begin{array}{ll}\text { Prix : rel. sur papier blane. } & 43 \text { in } \\ \text { - sur papier chine. } & 36 \\ \text { - colorie. } & 80\end{array}$

ART DE CULTIVEX LES JARDINS, ou Annuairodu bon Jardinier et de l'Agronome, renfermant un calendrier indiquant, mois par mois, tous les travaux à fairo tant en jardinage qu'en agriculture: les principez générauz du jardinage; la culture el la description de toutes les espèces o: variétés de plantes polagères, ainsi que toutes les sspèces et variétés de plautes utiles ou d'agrément; par un Jardinier agronome, 1 gros vol. in-18.1843. Orné de figures. 3 fr. 30 ART (l') DE FAIRE LES VINS DE FRUITS, précédé d'nne Esquisse bistorique de l'Art de faire le Vin de Raisin . de la manière de soigner une cave; suivi de ''Art de fairo le Cidre, le Poiré, les Aromes, le Sirop et le Sucre de Pommes de terre, etc ; trajuit de l'anglais, de Accou, par MM. $G^{k \star}$ of $\mathrm{OL}^{* * *}$. UL $\mathrm{vol}$. in $S$ avec planches.

ASSOLEMINTS, IACHERES ET SUCCESSION DES CULTURES, par feu V. YVART, annoté par M. V Rerwo, inspecteur de lagriculture. 3 vol. in-18. $\$ 0$ fr. 0 Ideris. Edition en 1 rol. in-4. $12 \mathrm{fs}$. Quvrage contenant les méthodes usitées en Lungleterre, en Allemagne, en Italie, en Suisse et en France.

BOžrTER (le nouveau), su Traité des Maladies des Bestiaux, Description raisonnée de leurs maladies el de lou straitement, par MI. DeLAGUETT, médecin-vétér. In-12. J Ir. 30 BOCCHERIE TAXEE, ou Tableau figuratif de toutes les catégoriss.

$75 \mathrm{c}$.

CALENDRIER DU BOE CULTIVATEUE, ou Manuel de l'Agriculteur-Praticien, par C.-J.-A. Mf ATyIE Doy 4 fr. $3 \mathrm{C}$ 
CHASSEUR-TAUPIER (le), ou l'Art de prendre les taupes par des moyens sùrs et facles, précédé de leur histoire naturelle, par M. RÉDAREs. in-18, fig. 90 cent.

CODE EORESTIER, conféré et mis en rapport avec la législation qui régit les différents propriétaires of usagers dans les bois, par Mu Cusson. 2 vol. in-8. 19 fr.

CORRESPONDANCE RURALE, contenant des observations critiqques et utiles, par DR kA BreTONNaRIR. 3 vol. in-12.

7 ir. 50

CORDON BLEU (le), nourelié Cuisinièrobourgeoise, ródigée et mise par ordre alphabétique, par Mlle Marguruitr, $12^{\circ}$ édition, considérablement augmentée. In-18. $1 \mathrm{fr}$.

COURS SLEMENTAIRE D'AGKICULTURE, par M. RISLER. In-12. 2 fr.

COURS COMPLET D'AGRICULTURE (noureau), du 19 siècle, contenant la grande ot la petite culture, l'économie rurale domestique, la médecine véiérinaire, etca, par lss Membres de la section d'Agriculture de l'Institut de France, etc. Nouvelle éditiun revue, corrigée et augmentée. Paris, Deterville. 16 vol. in-8, de près de 600 pages chacun, ornés de planches en taille-douce. $56 \mathrm{fr}$.

- D'AGRICUL TURE (petit), ou Encyciopédie agricole, par M. MaUNy de Moráay, contenant les livres du Cultivateur, du Jardinier, du Forestier, du Vigneron, de l'Econo. mie et Administration rurales, du Proprietaire et de 'Eleveur d'animaux domestiques. 7 vol. grand in-18, a vec fig. $1.5 \mathrm{f} .50$

COURS CONPLET D'AGRICULTURE PRATIQUE, par BURger, PFEIL, ROHLWES et RUFFINY; trad. de l'sll. par N. Nonnot; suivi d'un Traité sur les Vers à Soie et la Culture du Murier, par M. Borafods, etc. In-4. $10 \mathrm{fr}$.

- SIMPLIFIÉ B'AGRICULTURE, par L. DURoIs (Voyez Encyciopédie du Cultirateur). 9 vol. in-12. 20 fr.

* CULTIVATEUR (le) ANGLAIS, ou OEurres choisies d'Agriculture et d'Economie rurale et politique, par ARTRUR YoUkG. 18 vol. in-8.

DICTIONNAIRE D'AGRICULTURE RATIQUE contenant la grande et la petite culture, par M. lo comte Frangors de Neufchatead. 2 vol. in-3.

$12 \mathrm{f}$.

"DICTIONNAIRE NES JARDINIERS, ourrggo traduit de l'anglais de MILLER. $10 \mathrm{vol}$. in-4.

50 fr.

"DICTIONNAIRE RURAL ET RAISONNÉ des plantes préservatives et curalives des Maladies des Bestiaus, par Mme GaCoN-Defour. 2 vol. in-8. $6 \mathrm{fr}$. ÉCOLE DU JARDIN POTAGER, suivio du Traité de 
la Culture des fêchers, par M. DE CoMBLEs, $6^{\circ}$ édition, revue par M. Lovis Duzors. 3 vol. in-12.

1. fr. 70

CCUSSON-GREFFE, ou nouvelle manière d'écussonner les ligneux, par VergNaUd RomagivésI. 1830. in-12.1 fr.

ELOGE HISTORIQUE de l'A bbé FrAIrcoIs ROZIER, restaurateur de 1 Agriculture française, par $\mathbf{A}$. TuiźBADT DE BERNEAUD. in-8.

1 fr. 80

零 ENCYCLOPÉDIE DU CULTIVATEUR, ou Cours complet et simplifié d'agriculture, d'économie rurale et domes tique, par M. Joors DuboIs. $2^{\circ}$ édition, 9 vol. in-12 ornés de gravures.

Le vol. I se verid sẻparément $20 \mathrm{fr}$.

Cet ouvrage, très-simplifí, ssi indispensablo ause personnes qui ne vouuraient pas acquérir le grand ouvrage intitule : Cours d'agriculture au $\mathbf{X I X}^{\circ}$ siècle.

FABRICATION DU FROMAGE, par le Dr Fe Ga, traduit de l'italien par V. RENDU. in-8, fig. (Couronmé par la Société royale et centrale d'agriculture.)

gis.

GREFFES (Des) ET DES BOUTURES FORCÉS pour la rapide Multiplication des Roses rares et nouvelles, par M. Lolseleur Deslongchamps. in-8. (Extrait de 'Agriculteur praticien.)

30 c.

HISTOIRE DU PÊGHER, par M. DUVAL, in-8. 1 fr. 30 HISTOIRE DU POIRIER (Pyrus sylvestris), par DUVAL. Br. in-8 $8^{\circ}$ (extrait de l'A griculteur praticien).

1 fr. 50

GISTOIRE DU POMMIER, par.M. DUVAL.In-8. 1 fr. 30 INSTRUCTION SUR LE CHOU MARIN, par ROUSSRLOT, in-8.

LA TOMATE, 50 \&. $23 \mathrm{c}$.

INSTRUCTION PRATIQUE SUR LA PLA TTATION DES ASPERGES, par Bossin. Br. in-80.

$25 \mathrm{c}$.

SOURNAL W'ARICULTURE, d'Economio ruralo et

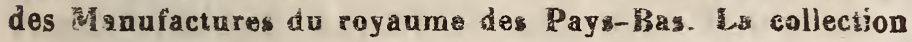
complète, jusqu'à la fin do $\$ 825$, se compose de 16 rol. in-8. Prix, Paris.

73 th.

JOURYAL DE MÉDECINE VETERINAIR E théorique et pratique, et Analyse raisonnée de tous les ouvrages français et étrangers qui on! du rapport avec la médecine des animaux aomestiques; recueil publié par MH. BRACYClark, Grípin, Gruzel, Delaguette, Dupuy, Godine 
jeune, Lebas, Princr, Rodet, médecins vétérinaires. 6 vol. in -8 . (1830) के 1835 .

Chaque année séparée.

$12 \mathrm{fr}$.

AISON DES CAAMPS (la) ou Manuel du Cnltivateur. par M. D. Pfluguer. 4 vol. in- 8 .

$15 \mathrm{fr}$.

"MAISON RUSTIQUE (la nouvelle), ou Économie ruralepratique des biens de campagne. 3 vol. in-4. fig. $24 \mathrm{fr}$.

MANUEL POPULAIRE D'AGRICULTURE, d'après l'ètat actuel des progrès dans la culture des champs, des prairies, de la vigne, des arbres fruitiers; dans l'édunation du gros bétail, etc., par J. A. SchuIPF; trad. de l'All. par NAPOLÉON NICELÈS. 1844. In-8.

$4 \mathrm{fr}$.

MANUEL DES INSTRUMENTS D'AGRICULTURE PT DE JARDINAGE les plus modernes, contenunt la graTure et la description détaillée des Instruments nouvelltement inventés ou perfectionnés, la plupart dessinés dans les meilleurs Ateliers de la capitale. Ourrage orné de 121 planches et de gravures sur bois intercalées dans le texte, par $\mathbf{M}$. BorTARD. 1 rol. grand in $-8^{\circ}$.

$12 \mathrm{fr}$.

MANUEL COMPLET DU JARDINIER, Maraîcher, Pépiniériste, Botaniste, Fleuriste et Paysagiste, par M. NoISETTE. 2A édition. 5 vol. in-8. $30 \mathrm{fr}$.

MANUEL DU FABRICANT D'ENGRAIS, ou de l'In. fluence du noir animal sur la végétation, par M. BERTIK. 1 rol. in-18.

2 fr. 30

MANUEL DU PLANTEUR. Du Reboisemet, de sa nécessité et des méthodes pour l'opérer, par DE BAzEzAlrE. In-12.

1 fr. 25

MELON (Du) ET DE SA CULTURE, par M. DUVAL. Brochure in-8. (Extrait de l'A griculteur praticien.) $75 \mathrm{c}$. MEMOIRE SUR LALTERNANCE DES ESSENCES FORESTIERES, par GUSTAVE GAND. In-8. 1 fr. 50 MÉTHODE ABREGE DU DRESSAGE DES CHEYUX DIFFICILES, et particulièrement des Cíhevaux d'armes. In-8.

2 fr.

MEMOIRE SUR LES DAHLIAS, leur culture, leurs propriétés économiques et leurs usages comme plantes d'or-

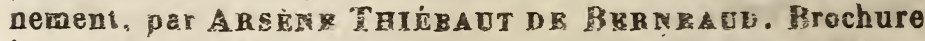
in- $\varepsilon$, g, ǵduicn.

$75 \mathrm{c}$.

METHODE DE LA CULTURE DT MELON en pleine terre, par M. J.-F. NoGET. in-8.

1 fr. 25

MONOGRAPHIE DU MELON, contenant la Culture, la Description et le Classement de toutes les variétés de 
celte espèce, etc., par M. JACQUIN aîné, 1 volume in-80 arec planches : Figures coloriées,

Figures noires,

$15 \mathrm{fr}$.

7 fr. 50

NOTICE SUR LA PLEUROPNEUMONIE ÉPIZOOTIQUE DE L'ESPÈCE BOVINE, régnant dans lo département du Nord, par A. B. LoIset, 1 vol. in-8 . 2 fr:

ORDONNANCE DE LOUIS XIV, roi de France el de Navarre, indispensable à tous les marchands de bois flottés, de charbon, à tous autres marchands et à ious les propriétaires de biens situés près des rivières navigables.in-18. 2 fr'

PARFAIT CONSERVATEUR DES GRAINS ET FARINES, par PERRET. Br. in-8.

$1 \mathrm{fr}$.

PATHOLOGIE GANINE, on Traité des Maladies des Ghiens, conterant aussi une dissertation très-détaillés sur la rage, la manière d'élever et de soigner les chiens; par M. Dela bére-Blainz, traduit de l'anglais et annoté par M. V. Delaguette, vétérinaire. A vec 2 planche représentant 18 espèces de chiens. 1 vol. in-8.

$6 \mathrm{fr}$.

PHARMACOPÉE VÉTÉRINAIRE, ou Nonvelie Phasmacie hippiatrique, contenant une classification des médicaments, les moyens de les préparer et l'indication de leur em: ploi, etc., par M. Bracy-Crark. 1 vol. in-12, plauches. 2 f.

PRATICIEN DE LA VILLE ET DE LA CAMPAGNE, par L. Hoste. 1 vol. in-12.

2 fr. 30.

PRATIQUE DU JARDINAGE, par ROGER SGHABOL.

2 rol. in-12, fig.

7 fr. 30

PRATIQUE RAISONNÉE de la taille du pêcher èn espalier carré, par LEPÈRE. in-8. Figures.

4 is.

PRATIQUE SIMPLIFIÉE DU JARDINAGE, à I'usage des personnes qui cultivent elies-mêmes un petit domaine, contenant un potager, une pépinière, un verger, des espaliers, un jardin paysager, des serres. des orangeries et un parterre, etc.; 60 édition; par M. L. Desols. 1 yol. in-18, orné do planches.

2 fr. 30

PREMIERES NOTIONS DE VITICULTURE, par STOLTZ. 1 vol. in-18.

$90 \mathrm{c}$.

PRINCIPES D'AGRICULTURE et d'Hygiène-Vétérinaire, par MAGNB. 1 rol. in-8.

$10 \mathrm{fz}$

BECUEIL DE MEMOIRES, notices et procédéz choisis sur l'agriculture,l'industrie, l'économie domestique, le mû rier multicaule, atc. (ou l'Omnibus journal, nnée 1834. 1 \% in-8.3ffr.

SEGRETS DE LA GHASSE AUX OISEAUX, cOn- 
tenant la manière do fabriquer les filets, les divers pièges, appeaux, etc.; l'art de les élever, de les soigner, de les guérir, etc., par M. G..., amateur. 1 vol. in-18 avec figures. 2 fr. 50

SERRES CHAUDES, Galerie de Minéralogie et de Géolozie, ou Notice sur les constructions du Muséum d'Histoire Naturelle, par M. Rogadlt (architecte). In-folio, $30 \mathrm{fr}$.

'SYSTEM OF AGRICULTURE, trom the Encyclopedia britannica, seventh edition, by JAMES CLEGHORR. Edimburgh, 1831, in-4, fig.

13 fr. 30

TABLEAUX DE LA PIE RURALE, ou l'Agriculture enseignée d'une manière dramatique, par M. DeSOJ EAUX. 3 rol. in-8.

$18 \mathrm{fr}$.

* THÉ ATRE D'AGRICULTURE ei ménage des champs; d'GLIVIER DE SERRES, nouv. édition. 2 vol. in-4. 25 fr TRAITE DES ARBRES ET ARBUSTES que I'on cultive en pleine terre en Europe et particulièrement en Irance, par Duhamel du Monceau, rédigé par MM. VeilGard, Jaume Saint.Hilaire, Mirbel, Poiret, et continuẻ par M. Loiseleur-Deslonchamps; ouvrage enrichi de 500 planches gravées par les plus habiles artistes, d'après les lessins de Ricdouté et Bessa, peintres du muséum d'bistoire gaturelle; $7 \mathrm{vol}$. in-fol., papier jésus vélin, figures coloriées. A u lieu de 3,300 francs,

$750 \mathrm{fs}$.

- Le même, papier carré vélin, figures coloriées. Au lieu de 2,100 francs,

- Le même, papier carrè fin, figures coloriées. $350 \mathrm{fr}$.

- Le même, figures noires. Au lieu de $775 \mathrm{fr} .200 \mathrm{fr}$.

\section{On a extrait de cet ouvrage le suivant :}

ROUVEAD TRAITE-DES ARRRES FRUITIERS, par DUHAME, nouvelle édition, très-augmentée par MM. Villlari, De Mrrbel, Poiret et Loiseleuk-DrslonCBAMPS, 2 rol. in-folio, ornés de 145 placeches. Prix:

Fig. noires $50 \mathrm{fr} . ;-$ fig. coloriées, papier fin. $100 \mathrm{fr}$.

Fig. coloriées, papier vélin.

$125 \mathrm{fr}$.

Fig. coloriées, format jésus vélin. . . $150 \mathrm{fr}$.

TRAITE DE CULTURE THÉORIQUE ET PRATIQUE, par HJBERT CaRRÉ. In-12.

2 fr.

TRAITE DE CULTURE FORESTIERE, par HERRI CotTA, traduit de l'dlemand par GUSTAYE GAJE, gardo général des forêts. 1 rol. in-8.

$7 \mathrm{fr}$. 
TRAITÉ D'INSTRUMENTS ARATOIREE, paz Meysen. Br. in-8.

- THAITE PAREAIT DES MOULINS, ou Recherches oxactes de toutes sortes de moulins connus jusqu'à présent, par L.-V. Natiros, J. Polly et C.-V. VonRRR. Am: stordam, 1734 (en hollandais), grand in-folio, fig. $75 \mathrm{fr}$.

TRAITE DE LA COMPTABILITE AGRICOLF, par "application du système complet des écritures en parties doubles, par MM. PERrade DE Jotedps père et fils. 4 cahiers in-folic.

12 fr.

TRA ITÉ COMPLET SUR LES ABEILLES, par l'abbé Della Rocca. $3 \mathrm{gr}$. vol, in-so.

TRATTE DE LAMENAGEMENT DES FORETS,

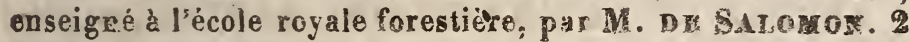
$\mathrm{val}$. in -8 et Allas in-4.

TRAITE DES MALADIES DES BESTIAUX, OC Description raisonnée de leurs maladies et deleurtraitement; suivi d'un apercu sur les moyens do tirer des'bestiaux les produits les plus avantageux, par M. V. DELAg URTTE, vétérinaire. In-12.

$3 \mathrm{fr} .30$

TRAITE SUR LES CHA MPIGNONS DOMESTIQUES, par Persoon 1 vol. in-go.

$12 \mathrm{fr}$.

TRAITE DU CHANVRE DU PIEMONT, DE LA GRANDE ESPECE, sa culture, son rouissago et ses produits, par ReY, in-12.

1 Ir. 30

TRAITÉ SUR LA DISTILLATION DES POMMES DE TERRE, par Evarista Hourier. In-18. $1 \mathrm{fr} .50$ TRAITÉ RAISONNE SUR L'EDUCATION DU CHAT DOMESTIQUE, et du Traitement de Maladies, par M. $R^{\star \star \star} \cdot \ln -12$.

TRAITÉ DE LA CULTURE DES ARBRES FRUITIERS, par Poulet. Broch. in $-8^{\circ}$.

$75 \mathrm{c}$.

TRAI'T DE LA TAILLE DES ARBRES FRUITIFRS, contenant les Notions indispensables de Physiologio régéta!e; an précis raisonné de la multiplication, de la plantation et do la culture; les vrais principes de la taille, et leur application aux formes diverses que reçoivent les arbres fruitiers, avee les planches pour l'intelligence du texte, par M. DE BAVAY, le Vilvorde, 1 vol. in-8.

3 fr. 50

TRAITE THEORIQUE ET PRATIQUE sur Ia Culare des Grains, suivi de l'Art de faire le pain, par PAR= YHTIER, etc. 2 vol. in-8, fig.

$19 \mathrm{fr}$. 


\section{education, Morale, piété.}

ABREGE CHRONOLOGIQUE DE L'HISTOIRE DE RANCE, depuis les temps les plus anciens jusqu' nos jours, par H. EngelHARD, in-18, broché.

Ideme, cartonné.

$90 \mathrm{c}$.

ABREGE DE LA TABLE on do l'Bistoire poétique, par le P. JoUVENGY, in-18.

$1 \mathrm{fr} .80$

ABREGE DE LA GRAMMAIRE ALLEMANDE pour les élèves des cinquième et quatrièmo classes des collèges de France, par M. Marcos. In-12, broché. 1 fr, $3 ?$

ABRÉGE DE LA GRAMMAIRE LATINE (ou Méthode brévidoctive do prompt enseignement), par If. JUz. LIEN. 1841, in-12.

ABREG DE LA GRAMMAIRE DE FAILLE in 12.

$75 \mathrm{c}$

ABREGE DE L'HISTOIRE SAINTE, avec des prew. ves de la religion, par demandes et par réponses, in-12. 60

ABRÉGE D'HISTOIRE UNIVERSELLE; premièn partie, comprenant l'histoire de Juifs, des Assyriens, de Perses, des Egyptiens et des Grecs, jusqu'à la mort d'Alexan dre--le-Grand, avec des tableaux de synchronismes, par $M$ Bodrgor, professeur de l'Académie de Besançon. 2e édi tion. In-12.

- Deuxieme partie, comprenant l'histoire des Romain dopuis la fondation do Rome, et celle de tous les peupl principaux, depuis la mort d'Alexandre-le-Grand jusqu l'avènement d'Auguste à l'empire, par M. BovBgor, et In-12.

- Troisième partie, comprenant UR A sRÉG DE l'HI: TOIR RE L'EMPIRE RONAIN, depuis sá fondation jusqu la prise de Constantinople, par M.BovrGON. In-12. 2 fr.?

Quairième partie, comprenant l'kistoire des Gaulois, I Gallo-Romains, lez Francs et les Français jusqu'à nos jous a vec des tableaux de synchronismes, par M. J.-J. BourGO 2 vol. in -12.

ABREGE DU COURS DE LITTERATURE do I

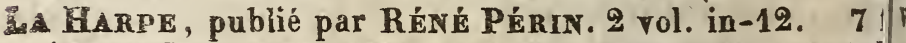

ANACISE DES TRADITIONS RELIGIEUSES pouples undigènes de l'Amérique, in-3.

ANNEE AFFECTIVE $\left(1^{\prime}\right)$, ou Sontiment: sur l'amo dle 
He Dieu, tirés du Cantique des Cantiques, pour chaque jour le l'année, par le Père A vrillon, in-12.

$2 \mathrm{fr} .50$

ARITHMÉTIQUE DES DEMOISELIES, ou Cours élóment. d'arithm. en 12 leç., par M. Yantenac.In-12. I fr.s0 Cahier de questions pour le même ourrage. $50 \mathrm{c}$. ARITHMÉTIQUE DES ÉCOLES PRIMAIRES, en 22 leçons, par L.-J. GEORGE, In-8.

I. I.

ARITHMETIOUE ELEMENTAIRE, théorique et pralique, par M. JuUANro, In-8.

$3 \mathrm{fr} .50$

ART DE BRODER, ou Recueil de modèles coloriés, analogues aux différentes parties de cet art, à l'usage des demoiselles, par UGDSTIN LEGRAND. 1 vol. oblong. $7 \mathrm{fr}$.

AS'TRONOMIE DES DEMOISELLES, or Entretiens, enire un frère ot sa sceur, sur la Mécanique céleste, démone trée et rendue sensible sans le secours des mathématiques, suivie de problèmes dont la solution est aisée, par JAMES FERGSSON etM. QUÉTRIN. 1 vol. in-12.

5 fr. 30

L'ASTRONOMIE ILLUSTRÉE, par ASA SMITH, revoe par Wagner; Wost at SARRUs. In-4 cartonné. 6 fr.

ATLAS (NOUVEL) NATIONAL DE LA RANCE, por départements, divisés en arrondissements et cantons, a ree le tracé des routes royales et départementales, des canaux, rivières, cours d'eau navigables, des chemins de fer construits et projelés, etc., dressé à l'échelle de 11,350,000, par

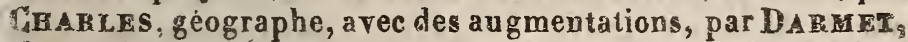
chargé des travaux topographiques au ministère des affaires étrangères. In-folio, grand-raisin de Vosges

Le Nouvel Atlas rational se compose de 80 planches (à cause de l'uniformité des échelles; sept feuillos contiennent deux départements).

Chaque carte séparée, en noir.

ace.

dem, coloriée.

$60 \mathrm{c}$.

AVENTURES DE ROBINSON CRUSOR, PaF MANIEL DE Fó́, édition mignone, $4 \mathrm{vol}$. in-32.

5 fro

AVIS AUX HARENTS nur la nourelle méthode de l'enseignement mutuel, par G. C. Herpin. In-12. $2 \mathrm{fr} .50$

BEAUX TRAITS DU JEUNR AGF, par A,-Fo- IREVILLE. Iń-12.

3 f.

CARIERS DE CFIMIE, l'usage de scoles st des Gen du monde, par M. Bubnotr. Prix, l'ouvrage comdlet, 4 cahiers in-12. 
CATECHSME du diocèse do Toul, qui doit ètro enseí gné danz toutes les écoles. in-12.

1 fr. 2 :

HISTORIQUE, par FLEURY . 1822, in-18. 50 HISTORIQUE (Petit), contenant, en abrégé, l'His toire saidele, par M. FueUry, in-18. Au Mans, 1838. $50 \mathrm{c}$ -so ou Abrégé do la Foi. in-18.

$30 \mathrm{c}$

CHOIX (Nouveau) D'ANECDOTES ANCIENNES ET MODERNES, tirées des meilleurs auteurs, contenant les faits les plus intéressants de l'histoire en général; les exploit: des héros, traits d'esprit, saillies ingénieuses, bon mots etc., otc. so édition, par Mme Celnart. 4 vol. in-18, ornés de julies rignettes. (Même ouvrage que lo Manuel aneodotique.)

$7 \mathrm{ft}$.

CHOIX DRECTURES ALLEMANDES, par STOEBER: In-8, In-8, 2a parlie.

$1 \mathrm{fr} .50$

$1 \mathrm{fr} .7 \mathrm{~s}$

CICERONIS (M. T.) ORATOR. Nova oditio, ad usum scholarum Tulli-Leucorum, 1823 ; in-18.

COMPOSitions MathématiQues, ou Problèmes géométriques et trigonométriques, à l'usage des écoles, In-8, par EscouBis.

2 fr. 25

COURS COMPLET, THEORIQUE ET PRATIQUE, D'ARITHMETIQUE, par RIVAIL. $3^{\odot}$ éd., in-12. 2 fr. 25

- Solutious. In-12.

$\$ 0 \mathrm{c}$.

COURS D'ARITHMÉTIQUE PRATIQUE, a l'usage des écoles primaires des deux sexes et des pères de famille, par \$. Molzert. In-18. 19r cahier, Connaissance des ohiffres.

20 cahier,

$3^{\theta}$ cahier, racions, Nombres, etc. $40 \mathrm{c.}$

Livrei des solutions.

NOUVEAU COURS RAISONNE DE DESSIN INDUSTRIEL appliqué principalement á la mécanique es à l'architecture, etc., par ARMERGAUd aîné, ARMENGAUn jeune et AMOURodx. 1 vol. grand in-80 et un atlas de 45 planches in -folio.

$25 \mathrm{fr}$. - DE THEMES, pour l'enseignement de la traduction du français en allemand dans les collèges de France, renfermarri un Guide de conversation, un Guide de correspondance, ei des Thêmes pour les élèves des classes élémentaires supérieures. 1 vol. in-12 broché.

COURS DE THÈMES pour les sixième, cinquième, quatrième, troisième et deuxième classes, à l'usage des col- 


\section{$-59-$}

bges, par M. Planche, professeur de rhétorique au cole bge royal de Bourbon, et M. Carpentier. Ouvragerecominandé pour les collèges par le Conseil de l'Université. $2 \mathrm{e}$ éd., ntièrement refondue et augmentée. 5 vol. in- $12.10 \mathrm{fr}$

A rec les corrigés à l'usage des maîtres. 10 vol. 20 \{r. 50

\section{On verd séparément:}

Lours de sixièmo à l'usage des élèves.

Lo corrigé à l'usage des maîtres:

2 fr.

Cours de $5^{\circ}$ à l'usage des élèves. \& fr. Le corrigé. 2 f 1 . 50

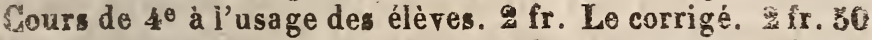

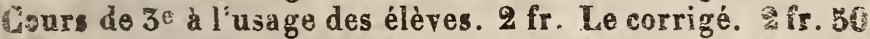
Cours de ze à l'usage des élèvez. \& fr. Le corrigé. \& fr. 50 DEVOTION PRATIQUE aux sept principau mystères louloureux de la très-sainte Vierge, mère de Dieu, In-12. 2 fr.

DIALOGUES ANGLAIs, ou Flements de la Converation anglaise, par PERRiN. In-12.

$1 \mathrm{fr} .9 \mathrm{~g}$

DIALOGUES MORAUX, instructifs et amusants, 'a l'uage de la jeunesse chrétienne. In-18. 1 fr.

DICTIONNAIRE D'ÉDUCATION MORALE, DE CIENCE ET DE LITTÉRATURE, par P. CAPELle. vol. in $8^{\circ}$ reliés.

$12 \mathrm{fr}$.

DICTIONNAIRE (Nouveau) DE POCHE français-2n. ;lais et anglais-français, par NJGENT ; revu par I. - F. FAIR : vol. in-12 carré.

是 $\mathrm{fr}$.

TDUCATION (Do I') DES JEUNRS PERSONNES, ou ndication de quelques améliorations importantes a introdnire lans les pensionnats, par Mlle FAURE. In-12.

$1 \mathrm{fr} .50$

ELÉMENTS (Premiers) D'ARITHMETIQUE, suivis l'exemples raisonnés en forme d'anecdotes, à l'usage de la ouresse, par un membre de l'Université. Ini-12. 1 î. 50

ELEMENTS DE LA GRAMMAIRE TRANCAISE, 3. LHomond. Ed. ref., p. L. GILBERT; $2^{\circ}$ éd.in-12. 75 . - (Nouveaux) DE LA GRAMMAIRE FRANGAIST, Jar M. FELLens. 1 vol. in-12.

1 f 25

ELEMENTS DE GRAMMAIRE HEBRAIQUE, PAT GYMAF, in 8. Cé. (Edition allemande).

6 fr. 50

Le même, in-8. Cé. (Edition française).

4 fr 50

ENSEIGNEMENT (1'), par MM. BERTADD-JULZTER: locteur ès-lettres, licencié ès-sciences, et C. PIPIDAD; locteur ès-lettres, bachelier ès-sciences. 1 gros vol. in-\$ do ¡00 pages.

\&

Cet ouvrage est indispensable à tous ceux qui veulent s'oc- 
cuper ayec intelligenco des questions d'éducation, traiter i fond les points les plus difficiles ot les moins connus de cettr. science difficile.

EPITRES RT EVANGILES des dimanchos ot fêtes do l'année. In-12.

\pm fr. $5 \mathrm{C}$

ESSAIS DE GEOMETRIE APPLIQUEK . par P. LEPELLETIER. In-8.

$4 \mathrm{fr}$.

ESSAI D'UNITE LINGUISTYQUE, pa: Jos. BoUZERAN. In-8.

$1 \mathrm{fr} .50$

ESSAI SUR LA GRAMMAIRE du langage natureí des sigfses, à l'usage des instiluteurs de sourds-muets, arec planches et figures, par RÉMI-VALADE, in-80.

2 fr.

ETRENNES DE L'ENEANCE, petites lectures illustrées, à l'usàge des Ecoles de Sourds-Mueis et des Salles d'Asile, par M. Valade Gabel. 1 rol.

1 fr. 80.

CTUDES ANALYTIQUES SUR LES DIVERSFS AC. CEPTIONS DES MOTE FRANGAIS, par MU FAJR. 1 тol. in-12.

2 ir. 3 ?

EXERCICES DE GRAMMAIRE ALLEMANDE, (thèmes et rersions), par SToEBER, in-12. Cé.

$73 \mathrm{c}$.

EXERCICES SUR ¿'ORTHOGRAPHE $I$ LA SINTAXE, calqués sur toutes les règles de la gramudire classique, par VILLEOY. In-12.

1 fr. 25

EXPLICATION DES EVANGILES DES DIMANCEES, par DE LA LUZRRNE. In-12, 5ै vo!.

$6 \mathrm{fr}$.

EXPOSE ELEMENTAIRE DE L.A TPEORIE DES INTEGRAIES DEFINIES, par A. MEYER, professeur à l'Universilé de Liége. 1 vol. in-80.

$10 \mathrm{fr}$.

FABLES JE FFNLON. Nour. édit. Llermont, 1838 , in -18

$30 \mathrm{c}$.

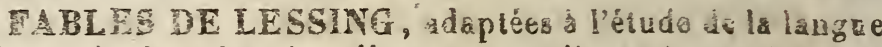
allomande daos les cinquième ê quatrième classes des collèges de France, mojennant un Vocabulaire ailemand-français, ure liste des formes irrégulières, l'indication de la cor. struction, ol les règles principales do la succession des mots, par Macus.

2 ir. 50

FLFGMIER. Horceaux choisis. In-18, avec portrait. 1 f. 80

FLEURE. Morceaux choisis. In-18, 2vec portrait. 1 f. 80

GEOSRAPHIE CLASSIQUE, suivio d'un Dictionnaire explicatif des lieux principaux do la géographio anciennc. par YiLE

1 fr. 25.

- DES ECOLES, par M. HOOT, continuateur do la 
Géographie de Malte-Brun el Guibal, ancien élèvo de l'Ecole polytechnique. 1 rol.

Átlas de la Géugraphie des Écoles.

1 fr. 38

2 fr. $3 c$

GÉOMÉTRIE PERSPECTIVE, avec ses applications a la recherche des ombres, par G.-H. DurodR, colonel du gé nio. In-8., arec un Allas de 22 planches in-4.

$\frac{1}{\mathrm{fr} .}$

GRADUS AD PARNASSUM, ou Nictionnaire tique latin-frânçais. In-8.

poé

GRAMMAIRE DE L'ENFANCE Clermont-Ferrand. 1839, in-12, cart.

GRAMMAIRE, on TRAITE COMPLET DE LA LANGUE ANGLAISE, par GiDOLPH. In-8. 5 ir

- CLASSIQUE, ou Cours complet et simplifí é de langz e française, par M. VileroY. In-12. 1 Ir. 25 Idem, Exercices.

- COMPLETE DE LA LANGUT ALLRMANDE, pour les élèves des classes supérieures des collèges de France, renfermant, de plus que les autres grammaires, un Traité complet de la succession des mots; un autre sur l'influence qu'elle exercée sur l'emploi de l'indicatif, du subjonctił. de l'infinitif et des participes; un Vocabulaire français-allemand des conjonctions et de locutions conjonctives; $p s$ s. MarCrs. 1 vol. in-12 broché

3 fr 50

GRAMMAIRE FRANCAISE à l'usage des pensionnuts de demoiselles, par Mme RovlleadX. In-12.

$80 \mathrm{c}$.

GRAMMAIRE (Nouvelle) ITALIENNE, réthudiq̨" et raisonnée, par le comte DE Frascolivi. In- 8 . $7 \mathrm{fr} .50$

GRAMMAIRE POLYGLOTTE, ou tableaux synop:iques comparés des langues française, a lemande, ang!aıse, italienne, espagnole et hébraïque, par Jost 1 v. in-8. $5 \mathrm{fr}$

GUIDE (Noureau) DES MËRES DE FAMLLLE, ов Education physique, morale et intellectuelle de l'wansac

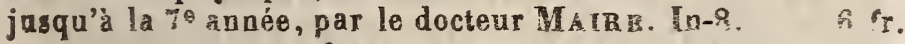

MISTOIRE ABRÉGE DU MOYEN-AGE, saivio d'u Tableau chronologique et ethnographique, pat Lenri LN GELEA EDT. In-8.

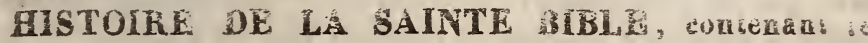
Vieux et le Nouveau Testament, par D RORACHONT. AU Mans, 1834; in -12 .

1 is

BISTOIRE DES FETES CIVILES ET RELIGIEUSES DE LA BELGIQUE MERIDIONALE, Jä M

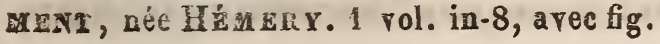

i 
HISTOIRE DES VARIATIONS DES ÉGLISES PROTESTANTES, par BOSSUET. 4 vol. in-8.

$18 \mathrm{fr}$.

IMITATION DE JÉSUS-CHRIST, avec une Pratiquo et uno Prière à la fin de chaque chapitre; traduite par le P. GONNELIEU. In-18.

1 fr. 73

INSTRUCTIONS POUR LA CONFIRMATION, à l' $\mathrm{Q}$ sage des jeunes gens qui se disposent à recevoir ce acromut, par l'abbé ReGNAULT. Toul, 1816, in-18. 75 .

IARDIN (le) DES RACINES GRECQUES, recreillies par LATCELOT, et mis en vers par LF MAISTRB DE SACY, par C. Bobet. In-8.

$5 \mathrm{fr}$.

JEUX DE CARTES HISTORIQUES, par H. JOUY; su nombre de 15, sur la Mythologie, la Géographie, la Chrow gologie, l'Astronomie, l'Histoire Sainte, l'Histoire Romaine, :Histoire de France, d'Angleterre, etc. - A 2 fr. chaque - La Géographie seule d̀ 2 fr. 50.

JUSTINI GISTORIARUM, ox Troge Pompeic, libri XLIV. Accedunt excerptiones chronologic ad usum scho= frgum. Tulli-Leucorum. 1823, in-18.

1 fr. 50

LECONS ELEMENTAIRES do Philosophie, destinǵes auz élèves do l'Université de France qui aspirent au grado de bachelier-è-lettres, par J.-S. FLOTTE. కo édition. 3 ₹. in -12 .

$7 \mathrm{fr} .50$

LEVES(des) A VOE, et du Dessin d'après nature, pa M Leglanc. In-18, figures.

MANUEL DE L'HISTOIRE DE FRANCE, par ACHMet D'Héricourt. 2 vol. in-8.

$13 \mathrm{fr}$.

MANUEL DES INSTITUTEURS ET DES INSPECTEURS D'ECOLES PRIMAIRES, par ${ }^{* * *}$. In-12. 1 fr.

MANUEL DE LECTURE, ou Méthode simplifiée pour apprendre à lire, par Pelletier. In-12 cart. $50 \mathrm{c}$.

MAPPEMONDE (la) de l'Atlas, de LESAG. $2 \mathrm{f}$.

METHODE COMPLETE DE CARSTAIRS, ditE AMER.GAINE, ou l'Art d'écrire en peu de leçons par des moyens prompts et faciles; traduit de l'anglais, sur la dernière édihas, par M. TRE HeRY, professeur.1 vol. oblong, accompagné d'un grand nombre de modèles mis en français. 's f. MÉTHODE NOUVELLE POUR LE CALCUL DES INTÉRÊTS à tous les Taux, par PIJon. In-18. i fr. 50 MÉTHODE POUR ENSEIGNER AUX SOURDSMUETS la langue française sans l'intermédiaire du langage 
des signes, à la portée des instituteurs, par M. VALADRGABEL. 1 vol, grand in-8.

$6 \mathrm{fr}$.

MODFLES DE L'ENFANCE, par l'abbí Th. Perrin. In -32 .

$30 \mathrm{c}$.

MORALE DE L'ENFANCE, ou Quatrain: moraux, a la portée des Enfants, et rangés par ordre méthodique, par M. lo vicomte de MoreL-VINDÉ, pair de France et membre de l'Institut de France. 1 vol. in-16. (Adopté par la Société élémentaire, la Société des méthodes, etc.)

$1 \mathrm{fr}$.

- Le mêms, tout latin, traduction faite par M. Vicror LECLERC.

- Le même, latin-français en regard.

$1 \mathrm{fr}$.

2 . fr.

MORALE (la) EN ACTION, ou Choix de faits mémora bles et Anecdotes instructives. In-12.

$2 \mathrm{fr}$.

PARAFARAGARAMUS, or Croquignole ot sa famille. In $=18$.

1 fr. 23

PÉLERINAGE (le) DE DEUX SOEURS, COLOM BELLE ET VOLONTAIRETTE, vers Jérusalem. In-4. fig I fr. 75

PENSEES ET MAXIMES DE FENELON. $2 \mathrm{vol.}$ in-18, portrait.

: fr.

- DE J.-J. ROUSSEAU. 2 rol. in-18, portrait. 3 fr.

- DE VOLTAIRE. 2 vol.in-18, portrait.

PETITS PROVERBES DRAMATIQUES, l'usage des jeunes gens, par Victor Geolet. In-12. 2 fr, 50 PRASÉOLOGIE FRANÇAISE-ALLEMANDE, par Fries. 1 vol. in-12.

2 fr.

PHRÉNOLOGIE DES GENS DU MONDE. Leçons $p u$ bliques données à Mulhouse, par le dr A. Pḱnot. In-8. 7 fr. 50 PREMIERES PAGES DE L'HISTOIRE DU MONDR. Lioçons publiques, données à Mulhouse, par A. Pḱños. In-8.

7 fr. 30

PRINCIPES DE LITTÉRATURE, mis on harmonio avec la morale chrétienne, par J.-R. Pér inNes. In-8. 8 fr.

PRINCIPES DE PONCTUATION, fondés sur la naturo du langage écrit, par M. FREY. (Ouvrags approwod par l'Uxiversité.) 1 vol. in-12.

1 fr. 50

PRINCIPES GENERAUX ET RAISONNÉS DE LA GRAMMAIRE FRANÇAISE, par DE RESTAUT In12.

2 fr. 50

PROGRAMME D'UN COURS ELEMENTAIRE DE

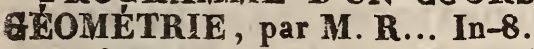

1 fr. 50

RECHERCHES SUR LA CONFESSION AURICD. LAIRE, par M. l'abbé GUILlois. In-12.

1 fr. 73 
RÉCRÉATIONS (Nourelles) PHYSIQUES ET MATHEMATIQUES, par GUYor. 3 vol. in- $8^{\circ}$ rel. $18 \mathrm{fr}$. RESUME DES PRINCIPES DE RHÉTORIQUE, par DE Blocka USEN. In-18.

$75 \mathrm{c}$.

RHÉTORIQUE FRANÇAISE, composéo pour l'instruction de la jeunesse, par M. Domainon. In-12. $3 \mathrm{fr}$.

RUUIMENTS DE L'HISTOIRE, en trois parties scolastiques, par M. Domairon, 3 vol. in-12. 9 fr.

RUDIMENTS DE LA LANGUE ALLEMANDE n par Eries. 1 rol. in-80.

$2 \mathrm{fr}$.

SAINTE (la) BIBLE. Paris, 1819, 7 rol. in-18., sur papier coquille.

$25 \mathrm{fr}$.

SAINTE BIBLE on Latiu of en Français, contenant l'Ancien et le Nouveau Testament, par DB GARRIikres. 10 rol. in--8.

$45 \mathrm{fr}$.

SCIENCE (ia)ENSEIGNÉE PAR LES JEUX, ou Théorie scientifique des jeux les plus usuels, accompagnée do recherche historiques sur leur origine, servant d'Introduction a l'étudo de la mécanique, de la physique, etc.; imitéo de l'anglais, par M. RichaRD, professeur de mathématiques. Ouvrage orné d'un grand nombre de vignettes gravées sur bois par M. GODARD. 2 jolis vol. in-18. (Même ourrage que le Hanuel des Jeux enseignant la science.)

$6 \mathrm{fr}$.

SELECT EF NOVO TESTAMENTO HISTORIAS ox Erasino desumpta. Tulli-Leucorum, 1823, in-18. 1f. 40 SERMONS DU PERE LENFANT, Prédicateur du roi Louis XV1. 8 gros vol. in-12, ornés de son portrait. 20 édition.

20 fr.

SIX (les) PREMIERS LIVRES DES FABLES DE LA FONTAINE, par VANDEREST. In-18.

$1 \mathrm{fr}$.

SYNONYMES ( Nouveaux) FRANCAIS à l'usage des demoiselies, par mademoiselle FauRE. 1 vol. in-12. 3 fr.

TABLEAU DE LA MISERICORDE DIVINE, tirée de l'Ecriture-Sainte, par l'abbé Bergier. In-12. 1 fr.

Id. Edition in-8, papier fin. $3 \mathrm{fr}$.

TABLEAUX (35) DE GRAMMAIRE FRANCCAISE, applicables à tous les modes d'enseignement, par M.J.-F. WALERT. In-folio.

3 fr. 50

TABLE DES VERBES IRREGULIERS de la langue allemande. Tours, in-8.

1 fr. 50

TABLES SYNCFRONISTIQUES DE L'HISTOIRB aniperselle, ancienne et moderne, par LAMP el ENGRLAARD. 1 rol. in-4 cartonæé.

5 fr. 
THE ELEMENTS OF ENGLISH GONVERSATION. by J. Perrin, in-12.

$1 \mathrm{fr} .73$

THE KEY, ou la traduction des thèmes de la grammaire anglaise de GIDOLPH. In-8.

$1 \mathrm{fr} .50$

TRAITÉ D'ARITHMÉTIQUE ET D'ALGĖBRE, par A. Bívilus. in-8.

$3 \mathrm{fr}$.

TRAITE DE L'ORTHOGRAPHE des Verbes réguliers, irréguliers et défectueux, par V.-A. Bodlenger. Paris, 1851 , in-18.

$30 \mathrm{c.}$

TRAITE DES PARTIGIPES, par Z. SHITS. In-12.30 c.

USAGE DE LA R IGLE LOGARITHMIQUE, ou Regle-calcul. In-18.

$25 \mathrm{c}$.

VERITABLE PERFECTION DU TRICOTAGE, br. in-12 par GAZYBOWSKA.

fr.

VOCABULAIRE USUEL DE LA LANGUE FRANCAAISE, par A. PeTER. In-12.

2. fr. 50

POYAGES DE GULLIVER. 401 . in-18, fig. 6 fr.

\section{OUVRAGES DE MIT̃. NOEL, GHAPSAE, PLANCHE PTELENS}

GAMMAIRT TATINE (nouvello) sur un plan trèsméthodique, par M. NoEL, inspecteur-général à l'Université, et $M$. FELLENs Ouvrage adopté par l'Université $1 \mathrm{fr} .80$ IXXKCICES (latins-français. 1 ir. 80 THEMES pour $7^{3}$ ot $80^{\circ}$. 1 fि 50 CORRIGES. ABREGE DE LA GRAMMAIRE TRANCAISE, par MM. NoEc of CHAPSAL. 1 rol. in-12.

EXERCICES íLEMENTAIRES, adaptẻs à l'abrégế de la Grammáire française de MM. Nort et Chapsat. i fr. GRAMMAIRE FRANCAISE (nouvelle) sur un plan très-méthodique, par MM. NoEz et CHAPSAL. 3 vol. in-12 qui se rendent séparément ${ }_{3}$ savoir:

- Le Gra

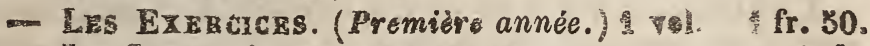

- L CORBIRE DES EXRECICES. 2 fr.

GXRCICES FRANCAIS SUPPLEMENTAIRES, sUs le difficultés qu'offre la syntaze, par M. Chapsal. Seconde annèe.) 1 vol. in-12. 
CORRIGÉ DES EXERGICES SUPPLÉMENTAIRES.

1 rol. in 12.

2 fr.

LECONS D'ANALYSE GRAMMATICALE, par MM. Nore et Chapsal. 1 rol. in-12.

$1 \mathrm{fr}, 80$.

IECOONS D'ANALYSE LOGIQUE, par MM. NOER et Gíapsal. 1 vol. in-12.

$1 \mathrm{fr} .80$.

TRAITÉ (nouveau) DES PARTICIPES, suivi de dic. tées progressives, par MM. Noel et Chapsat. 3 vol. in-12 qui se vendent séparément, savoir :

- Thérir des Partictres. 1 rul.

$2 \mathrm{fr}$.

- Exercices sdr les Participes. 1 rol.

$2 \mathrm{fr}$.

1 vol.

- CORRIGE DES EXRCICES SUR RE PARZICIPES.

SYNTAXE FRANĢAISE, par M. GHAFAL, \& l'usage des classes supérieures. 1 rol. in 12.

2 fr. 7 ?

COURS DE MYTHOLOGIK. 1 Fol. IE-1. $2 \mathrm{fr}$.

DICTIONNAIRE (noureau) DE LA LANGUR WRAN. CAISE. édition. rol. in-8, grand papier. Br. $8 \mathrm{fr}$. Cartonné, 8 fr. 75 c. Relié, basane.

9 fr. 25 c.

METHODE POUR L'APPLICATION IZS PRINCIPES DE LA GRAM hAIRE, par M. Chapsal. 1 vol. in-12.

$1 \mathrm{fr} .50$.

\section{OUVRAGES DL M. MORIN}

GEOGRAPHIE ELEMENTAIRE ancienne et moderno prócédée d'un Ábrégé d'astronomie. In-12, cart. $1 \mathrm{fr} .80$

OEUVRES DE VIRGILE, traduction nouvelle, avec le texte en regard ot des remarques. 3 vol. in-12. 7 fi 30. BUCOLIQUES ET GEORGIQUES. 1 rol. in-12. $2 \mathrm{fr} .30$. PRINCIPES RAISONNES DE LA LANGUE FRANCAISE, à l'usage des collèges. Nouv. éd. In-12. 1 fr. 20 - DE LA LANGUE LATINE, uirant la méthodo de Port-Royal, à l'usage des collèges. 1 rol. in-12. $1 \mathrm{fr} .23$. NOUVEAU SYLLABAIRE, ou Principes de lecture. Ouvrage adopté par l'Université, l'usage de écoles primaires.

60 .

TABLEAUX DE LECTURE destinés à l'enseignement mutuel ot simultané, 30 feuilles.

$\mathrm{s} \mathbf{f r}$.

ABREGÉ CHRONOZOGIQUE DES CONCILES GÉEERAUX, par GAOTIER. In-8

3 fr. $: 0$ 


\section{$-67-$ \\ OUVRAGES DIVRRS.}

ABUS (des) EN MATIEIR ECLLSIASTIQUE, par M. BOYARD. 1 vol. in-8.

ALBUM PHOTOGRAPHIQUE publié par livraisons, a 6 fr. chacune, par BLANQUART-EVRARD. V. page 84 .

ALPHABET DU TRAIT, Appliqué a la Menuiserie (Móthode élómentaire à l'aide de laquelie on peut apprendre le trait sans maîire), par J.-R.-R. DELAUNAX. 1 vol. grand in-8 ai 20 planches.

$10 \mathrm{fs}$.

ANIMAUX (les) PARLANTS, poèmo épique en 26 chants, de CAstr, traduit de l'italien par Man EGAAL. \& vole in-8.

$6 \mathrm{fr}$.

ANNALES DE L'INDUSTRIE NATIONALE ET ETRANGERE, par MM. LENORAND et DE MOÉER. 1820 à 1826. 24 vol. in-8, demi-rel.

$90 \mathrm{fr}$.

- Becdil. INDESTRIEL, Manufacturier, Agricole el Commercial, pas M. DE Molíor 1827 1831. 20 rol. in-8, cartonnés

$80 \mathrm{fr}$.

* ANNALES UES ARTS ET MANUFACTURES, par MM. OREILLY ol BARBIER-VERARS. 56 vol. in-8. $112 \mathrm{fr}$. ANNER FRANCAISE, on Mémorial des science", des Arts et des Lettres. 1825, $1^{\text {Is }}$ année. 1 vol. in $=8$. $7 \mathrm{fr}$. $-1826,28$ année. 2. vol. in-8.

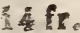

ALMANACH ENCYCLOPEDIQUE Récréatif ot Populairo, pour 18591 vol. in-16, grand-raisia, orné de jolies gravures.

$30 \mathrm{c}$.

L. os annéer 1840 * 1859 sendent chacrnis

$60 \mathrm{c}$.

ANTIGONE, par BALLANCHE. 1 vol. in-8 orné de ses gravures, d'après les dessins de Bovillon.

AQUARELLE - MINIATURR PERFCTIONNEE reflets métalliques et chatoyants , et peinture a l'huile sur velours, par M. SAIBT-VICTOR. 1 vol. grand in-8, orné de 15 planches, dont 7 peintes à la main.

$12 \mathrm{fr}$.

AQUARELLE (l'), ou les Fleurs peintes d'après la méthode de M. Redouté, par M. PASCAL, contenant des notions de botanique à l'usage des personnes qui peignent les fleurs, lo dessin et la peinturé d'après les madèles et la nature. In-40 orné de plánches noires et coloriées.

4 fr. 50

ARCHIVES DES DECOUVERTES 2 T DES INPENTIONS NOUVELLES faites dans les Sciences, les 
Arts et les Manufactures, on France et à l'Etranger. Paris, 1808 à 1838.50 vol. in-8, rel.

$210 \mathrm{fr}$.

ARCHIVES (nouvelles) HISTORIQUES DES PAYS\$AS, ou Recueil pour la Géographie, la Statistique, l'Histoire, etc., par le baron DE REIFFENBERg. Juillet 1829 á mai 1851.9 numéros in-8.

$18 \mathrm{fr}$.

ART DE LAA GUERRE (I'), par principes et par règles, par De Puységur. 2 vol. in-40.

ART DU PEINTRE, DOREUR ET VERNISSEUR, par WATIN; $11^{\circ}$ édition entièrement refondue, par $\mathbf{M}$. BOURáols, architecle des Tuileries. 1 vol. in-8. 4 fr. 50

ART (l') DE CONSERVER ET D'AUGMENTER LA BEAUTE, corriger et déguiser les imperfections do la nature, par LAMI. 2 jolis vol. in--18, ornés de gravures. 6 fr.

- DE LEVER LES PLANS, et nouveau Traité d'ATpentage et de Nivellement, par Mastaikg. 1 vol.in-12. Nourelle édition.

$4 \mathrm{fr}$.

ART DE TRICOTER développé dans toute son étendue, ou Instruction complète et raisonnée sur loutes sortes de Tricotages simples et compliquéz, par MM. NetTo et LEHMANN. In-folio oblong.

$18 \mathrm{fr}$.

ART DU TYPOGRAPHE, par VINGARD. 1 vol. in-8, Q édition.

$6 \mathrm{fr}$.

ARTISTE (1') EN BATIMENTS. Ordres d'architecture, sonsoles, cartouches, décors et attributs, etc.; par L. Bra -

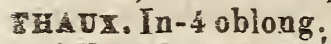

ATLAS TI MEMORIAL OR SAINTE-BWLEN ह

负 1 .

ATTENDS-MOI AD MONT-SAINT-MICHCL, par AFre Bradlès. Paris, $1840,2 \circ$ édition, in-8. $75 \mathrm{C}$.

BAREME A L'USAGE DES MARCHANDS DE CAFÉ. In-S.

BAREME DU LAYETIER, contenant le toisé par ro= figes de toutes les mesures do caisses, depuis 12-6-6, jusqu'à 72-72 72, etc., par BIEN-AIMÉ. 1 vol. in-12. i fr. 25

BESANCON : DESCRIPTION HISTORIQUE de MOnuments et Etablissements publics de cette ville, par A. GUÉNARd. In-18.

BIBLIOGRAPBIE ACADEMIOUE BELGE, ou Ropertoire systématique et asdalytiquje des mémoires, dissertasions, etc., publiés jusqu'à ce jour par l'ancienne of la nourelle Académie de Bruxelles, par P. NAmur. 1 rol. la-8.

3 fr. 
BIBLIOTHE்QUE CHOISIE DES PĖRES DE L'EGLISE grecque et latine, ou Cours d'Eloquence sacréo, par Mf.-N.-S. Gulllon. Paris, 1824 à 1828. 26 vol. in-3. dewi-rel.

80 in

\section{BIBLIOTHÈQUE DES ARTS ET MÉTIERS,}

Format in-18, grand papier:

GIVRE do l'ARPENTEUR-GEOMETRE, paE MAF. Place el Foucare, 1 vol.

If

- du RRASSEUR, par M. Drleschazer Pr:50

- de la COMPTABILITE DU PATMENT, par M. Digeon. 1 rol. $2 \mathrm{fr}$. - du CULTIVATEUT, pas M. Madr DE MORNAT. rol. 2 fr. 30 - de l'ÉCONOMIE et de l'ADMINISTRATION RUBALE, par M. DE MORNAY. 1 vol. 2 fr. 50 - du EORESTIER, par M. DE MonNay. 1 vol. 2 fr. - du JARDINIER, par M. DE Morvay. 2 vol. fí. - des LOGEURS el TRAITEURS. 1 vol. $1 \mathrm{fr} .30$ da MEUNIER; par M. DE MORNAT. 1 vol. 2 fr. 30 - du PROPRIÉTAIRE et de l'ELEVEUR D'ANI MAUX DOMESTIOUES, par RH. MORNAY. $2 \mathrm{fr} .50$ - מU FABRICANT DE SUCRE et du RAFINEUR, Jar M. DE MORNAY. 1 pol. - du TAILLEUR, par M. Augustir Caneva. 1 fr. 30 - du TOISEUR-VERIFICATEUR, par M. DgeON. rol.

2 fr.

- du VIGNERON et du FABRICANT DE CIDRP; Jar M. DE MonNay. 1 vol. $2 \mathrm{fr}$. Cette collection, publiée par les soin ie lik. itant devenue la propriété de M. Ronkt, c'est à ce dernier [ae MM. les libraires dépositaires de ces ouprage devront - endre compte des sxemplaires envoyé en commission par \$. Bagnerre.

BILAN EN PERSPECTIVE DES CAEMINS DE PIP en France; Envahissement du travail national per le nécanisme, par DAGREAU-SYMONSEN. In-8 2 fr. 25 BULLETIN DE LA SOCIÉTÉ D'ENCOURAGEUENT pour l'industrie nationale, publié avec l'approbation lu Ministre de l'Intérieur. An XI à 1858. 54 vol. in-4, ivec beaucoup de gravures. Prix de la collection. $729 \mathrm{fr}$. 
On rend séparément le année 1 à 28,9 fr.; 290 à 55 15 fr.; $56^{\circ}$ et $57^{\mathrm{e}}, 56 \mathrm{fr}$; table, 6 fr.; notice, 2 fr.

BULLETIN DE LA SOCIÉTE FRANGAISE D PEOTOGRAPHIE, Journal mensuel. $3^{s}$ année. Franc $12 \mathrm{fr}$ : Etranger.

15 i

CALCUL DES ESSIEUX pour les Chemins de Fe COUP-D'OEIL SUR LES ROUES DE WAGONS de ch mins de fer. Br. in-8.

CARACTERES POÉTIQUES, par ALLET. In-8. $6 \mathrm{f}$

CARTE TOPOGRAPHIQUE DE L'ILE SAINTE HELENE, dressée pour lo Mémerial de Sainte-Hélène. Ir plano.

CAUSES (des) DE LA DECADENCE DE LA PO LOGNE, paT D'HERBELOT. In-8.

$1 \mathrm{f}$

CHARTE (de la) D'UN PEUPLE LIBRE et digno $c$ la liberté, par A.-D. VkrgNadv. In-\$.

1 fr. 8

CHRIST, ou l'Affranchissement des Esclaves, Drame hy manitaire en cinq actes, par M. H. Cavel. In-8. 3 fr. CHIMIE APPLIQUEE AUX ARTS, par CHAPTAI membre de l'Institut. Nouvelle édition avec les additions d M. GUILLERY. 3 livraisons formąnt un gros volume in-? grand papier.

$20 \mathrm{fi}$

CHINE (la), L'OPIUM ET LES ANGLAIS, cont nant des documents hisioriques sur le commerce do Grande-Bretagne en Chine, etc., par M. SAURIN. I fi

CHOLERA (le) A MARSEILLB, en 1834-1835. In-8 Marseille, 1835.

CODE DES MAITRES DE PGSTE, des Entrepreneu de Diligences et de Roulage, e! des Voitures en général pa serre et par eau, ou Recueil général des Arrêts du Consei Arrêts de règlement, Lois, Décrets, Arrêtés, Ordonnances d roi ol autres actes de l'autorité publique atc., par M. LArrol a vocat à la Cour Impériale de Paris. 2 vol in-8. 12 fi

CODE DE LA PROPRIÉTE, par M. ToUsSAINT 2 vol. in- 8.

$15 \mathrm{fs}$

COLLECTION DE MANOELS.-RORET, formant wn Encyclopédie des Sciences et des Arts. 400 vol. in-18, are un grand nombre de planches gravées. (Voir le détail p. 3

COMPTES-FAITS des intérêts à 6 du cent par an, etc. par DUPONT aîné. In-12.

1 fr. 2

COMPTES-RENDUS HEBDOMADAIRES des séan ces de l'Académie des Sciences, par MM. les Secrétaire perpétuels. Paris, 1835 à 1858.47 rol. in-4 $4^{\circ}$ à $10 \mathrm{fr} .470$ fi 
CONCORDANCE DE L'ÉCRITURE-SAINTE, avec is traditions de l'Inde; par Ad. KARSTNER. In-8. 3 fr. CONDUITE (la) DE St-IGNAGE DE LOYOLA menant ae àme à la perfection, par le P.A. VAtier in-12. $1 \mathrm{fr} .75$ CONGRes SCIENTIFIQUE de France. Premièro Seson, tenue à Caen, en juillet 1853. Iu-8. 4 fr. 50 CONSIDÉRATIONS SUR LA PERSPECTIVE, par ENOIT DUPORTAIL. Br. in-8.

$1 \mathrm{fr} .25$.

CONSTRUCTION DES BOULONS, Ecrous, Harpons, lefs, Rondelles, Goupilles, Clavettes, Rivets et Equerres, iiv. de la construction des Vis d'Archimède, par A. C. ENOIT-DUPORTAIL. Br. in-8.

$3 \mathrm{fr}$. CONSTRUCTION ET EMPLOI DU MICROSCOPE, ar Hannover, traduit par Ch. Chevallier, in $-8^{\circ} .5 \mathrm{fr}$. CONSTRUCTION (do la) DES ENGRENAGES, el de meilleure forme à donner à leur denture, par S. HAINDz. a-12. Fig.

$4 \mathrm{fr}, 30$

DE LA CONTREFACON des curres artistiques, de Lodèles es des dessins de fabrique (législation et jurispruence). par lialmels, in-8.

$25 \mathrm{c}$. COUP-D'OEIL SUR LE THEATRE DE IA GUERRE l'ORIENT, trad. de l'allemand, de Wussow, par J. IARMIER. In-8.

$2 \mathrm{fr}$. LOUP-D'UELL GEERAL ET STETSTIQOE a la Iétallurgie considéréo dans ses rapports avec l'Industrio et 1 richesso des peuples, etc., par TH. VIRLET. In-8. 3 fr. COUR DE CASSATION, Loi thèglements, par M。 ABBi. 1 vol, in-8, grand format.

COURS ELAMENTAIRE DE DESSIN INDUS 'RIEL, la l'usage des écoles primaires, par ARMENGAUD înó, ARMENGAUD jeune, et LAMOUROUX. in-4 oblong. $8 \mathrm{f}$. COURS DE FILATURE DE COTON, par M. DRAPIER. a-8, avec appendice.

$\mathrm{s} \mathrm{fr}$.

COURS GRATUIT DE CHALEUR, appliqué aux Aris adustriels, 6 leçons ou cahiers, in-8, par BuREL. 2 fr. 40 COURS DE PEINTURE A L'AUUARELL, conteant des Notions générales sur le Dessin, les Couleurs, ete.; ar DUMÉNIL. In-18. $1 \mathrm{fr} .73$

COUTUME DU BAILLAGE DE TROYES, qrec les iommentaires de M. Lovis-LIs-Grard. Paris, 175\%, inolio. Relié.

$30 \mathrm{fr}$.

LE CURE INSTRUIT PAR L'EXPÉRIENCE, ow Tingt Ans de ministère dans une Paroisse de campagne, ar l'abbé AgUetTAND. 2 vol. in-12. $\quad 5 \mathrm{fr}$. 
CULTE (du) MOSAIQUE a zix siècle, par P.-I In.12.

DERNIERS MOMENTS DE LA RÉVOLUTION D. POLOGNE, en 1831, par M. JANOWSKI. In-8. j fï DESCRIPTION D'UN APPAREIL DESTINE A EVI TER LES DANGERS D'EMPOISONNEMENT dans I Fabrication du Fulminate de mercure, par G.-V.-P. Casas: DELON. In-8.

$40 \mathrm{c}$

* DESCRIPTION DES MACHINES et procédés spécifié dans les BREVETS D'INYENTION, de perfectionnement \& d'importation, dont la durée est expirée, publiée d'après le ordres du Ministre de l'Intérieur, par MM. Moland Ciristian, etc. 88 rol. in-4, a vec un grand nombre d planches gravées. Paris, 1912 à 1847. Les 88 vol. 12 fs 7 !

Chaque rolume se vend séparément: 1 or à 5 e 13 fr., 6 à 200 à 12 fr.; $21^{\circ}$ a $88^{\circ}$ à 15 fr.

- Table générale des matières contenues dane les pre mists rolumes. In-4.

DESCRIPTION DES MACHINES ET PROCÉDEE pour lesquels des brevets d'invention ont été pris sous It régime de la loi du 5 juillet 184 (nouvelle série). Tome 1 à 29. Chaque voluare

DESGRIPTION GENERALE DE LA CIINE, pEI l'abbś Grosier. 2 rol. in-8.

DETAILS SUR LA NAVIGATION AUX COTES DE SAINT-DOMINGUE et dans les débarquements. In-\$. $4 \mathrm{fr}$.

DICTIONNAIRE DES ARTS ET MANUF ACTURES, de l'agriculture, des mines, etc. Dascription des procédés de l'industrie française et étrangère Publié par B. LABodlaYa, 4 rol. in $8^{\circ}$, ou 2 très-forts in- $8^{\circ}$ grand raisin.

$60 \mathrm{fr}$.

- DICTIONYAIRE DES DÉCOUVERTES, Inventinn Innovations, Perfectionnements, etc., en France, dans les Seiences, la Littérature et les Arts, de 1789 à 1820. 17 roi. in-8. Demi-rei.

DICTIONNAIRE ÉCONOMIQUE, contenant dirers moyens d'augmenter sou bien et de conserver sa santé, par M. Noël CHomel. 4 vol. in-fol. reliés.

$50 \mathrm{fr}$.

DICTIONNAIRE DES GIROUETTES ou nos Contemporains peints par eux-mêmes. Paris, 1815 , in-8. 5 fr:

- DICTIONNAIRE TECHNOLOGIQUE, ou Noureau Dictionnaire universel des Arts et Métiers, et de l'économio industrielle et commerciale, par une Société de savants et d'artistes. Paris, 1822. 22 vol. in-8, et Allas in-4. $150 \mathrm{fr}$ 
DICTIONNAIRE UNIVERSEL géographique, statistique, historique et politique de la France. 5 vol. in $8.40 \mathrm{fr}$.

DICTIONNAIRE UNIVERSEL de la Géographie commerçante, par J. PEUcheT. 5 vol. in -4 reliés.

$40 \mathrm{fr}$.

DROITS DES PECHEURS à la ligne, par Moricead, br. in. 18.

$25 \mathrm{c}$.

DZIELA KRASICKIEGO, dziesiec Tomow W Jednym. Barbezata, in-18. (OEavres poétiques de Krasicki.) $25 \mathrm{fr}$.

ÉLETIONS (des) SELON LA CHARTE et les lois du royaume, par $M$. BoYARD. in -8 .

ELEMENTS OF ANATOMY GENERAL, special and comparative, by DAvid Craigie. Edimburg, 1831; in-4. figures.

$15 \mathrm{fr}$.

ELEONORE DE FIORETTI, ou Malheurs d'une jeune Romaine sous le pontificat de $\mathrm{d}^{\star \star \star} .2 \mathrm{vol}$. in 12.

ÉLOGE DE CHORON. Br. in. 8.

$3 \mathrm{fr}$.

2 fr. 59

DE LA FOLIE, par ERASME, traduction nouvelle, par C. B. de Panalbe. in-8.

$6 \mathrm{fr}$.

EMMELINE eT MARIE, suivies des Mémoires sur Madame Brunton; traduit de l'auglais. 4 vol. in-12. 6 fr.

EMPRISONNEMENT (de l') pour dettes. Considéra tions sur son origine, ses rapports avec la morale publique et les intérêts du commerce, des familles, de la société, suivies de la statistique générale de la contrainte par corps en France et en Angleterre, et de la statistique détaillée des prisons pour deltes de Paris et de Lyon, et de plusieurs autres grandes villes de France, par J.-B. BAYLE-ModiLLARD. Ouv. couronné en 1835 par l'Institut, in-8. $7 \mathrm{fr} .50$

ENCYCLOPEDIA BRITANNICA, or a Dictionnary of Arts, Sciences, and miscellaneous Literature. Edimhurgh, 20 vol. in-4, fig.

$300 \mathrm{fr}$.

ENTREE DE CHARLES-QUINT A ORLEANS, par VERGNAUD. In-8.

EPILEPSIE (de l') EN GENERAL, et particulièroment de celle qui est déterminé par M. Doussin-Dubredre. 1 vol. in-12, 20 édition. $3 \mathrm{fr}$ :

EPITAPIIE DES PARTIS; celui dit just -milieu, son avenir; par H. CAVEL. in-8.

$1 \mathrm{fr} .50$

ESPAGNE (de l') ET DE SES RELATIONS COMTERCIALES, par .-A. DE CH. in-8.

2 fr. 50

ESPRIT DE LA COMPTABILITE COMMERCIALE, ou Résumé des Principes généraux de Complabilité, paz VALENTIN MEYER-KOECHLIN. In-8.

2 fr. 50 
ESPRIT DES LOIS, par Montesquieu. 4 vol. in-12. $12 \mathrm{fr}$. ESQUISSE D'UN TABLEAU HISTORIQUE des progrès de l'esprit humain, par CoNDorCex. In-18. 3 fr.

ESSAI HISTORIQUE ET CRITIQUE SUR LES JOURNAUX BELGES, par A. WARzéE. $1^{\text {ro }}$ partie, Journaux politiques, in-8.

$3 \mathrm{fr}$.

ESSAI SUR L'ADMINISTRATION, par le Sous-Préfot de Béthune. In-8.

$3 \mathrm{fr}$.

ESSAI SUR L'AIR ATMOSPHÉRIQUE, par BRAINE, in-8.

$75 \mathrm{c}$.

ESSAI SUR LE COMMERCE et les intérêts de l'Espagne et de ses colonies, par F.-A. DE Christophoro D'A Valos. In-8.

2 fr. 50

ESSAI SUR LES ARTS et les Manufactures de l'empire d'Autriche, par MARCEL DE Serres. 5 vol. in 8. $12 \mathrm{fr}$.

ESSAI SUR LANALOGIE DES LANGUES, par HENNEQUIN. In-S.

$5 \mathrm{fr} .50$

ESSAI SUR L'HISTOIRE GENERALE DES MA'THÉMATIQUES, par Ch. BosSUT. 2 vol. in-8. $15 \mathrm{fr}$.

ETUDES SUR QUELQUES PRODUITS NATURELS applicables à la teinture, par ARrAudon. Br. in- $8^{\circ}$. 1 fr. 25

EVENEMENTS DE BRUXELLES ET DES AUTRES VILLES DU ROYAUME DES PAYS-BAS, dopuis le 25 août 1850 , précédés du Catéchisme du citojen belge et de chants patriotiques. 1 vol. in -18 .

1 fr. 25

EXAMEN CRITIQUE DES NOTATIONS MUSICALES, par RAYMONDI. In-12.

2 fr. 50

EXAMEN DU SALON DE 1827, arec cette epigrapho: Rien s'est beau que le vrai. 2 brochures in-8.

- Idem. de 183 ', par VergNaUd.

EXAMEN HISTORIQUE ÜE LA REVOLUTION ESFAGNOLE, suivi d'Observations sur l'esprit public, la soligion, etc., par ED. BLAQUiérE ; traduit de l'anglais par J.-C. $P^{* * *} \cdot 2$ vol. in-8.

$10 \mathrm{fr}$.

EXPEDITIONS DE CONSTANTINE, accompagnées de réflexions sur nos possessions d'Afrique, par Y. Devorsirs. In-8, fig.

2 fr. 50

EXPLIGATIONS DU MARÉCHAL CLAUZEL. In-8. 1857.

$3 \mathrm{fr}$.

EXTRAIT D'UN DISCOURS sur l'Origine, les Pro. grès et la Décadence du Pouroir temporel du Clergé, par 5. E. Mgr l'ancien Archevêque de T.., In-8. 2 fr. FXTRAITS TIRES D'UN JCURNAL ALLEMAND des- 
tiné à rendre compte de la législation et du droit, dans toute les contrées civilisées, par M.J.-J.DE SELLoN. In-8. $1 \mathrm{fr} .50$

FAMILLE DURANDEL (la), par DE BLOCRBAUSEN. 1 vol. in-12 cartonné, couv. dorée.

1 fr. 50

FASTES DE LA FRANCE, ou Tableaux chronologiques, synchroniques et géographiques de l'Histoire do France, par C. Mollié. 1841, in-fol.

FÉCONDATION ARTIFICIELLE ET ÉCLOSION DES OEUFS DE POISSONS, suivie de réflexions sur l'Icthyogénie, par le dr Haxo.

2 fr. 50

FER PUR (DU) et de ses dissolutions ou alliages, par JULLIEN, br. in-8.

FÊTE DE JEANNE D'ARC A ORLÉANS (1885), br. in-8, par VERGNAUD-ROMAGNÉSIE.

1 fr. 50

FILLE (la) D'UNE FEMME DE GENIE, traduit do l'anglais de madame Hofcard: 2 vol. in-12.

$4 \mathrm{fr}$.

FLEURS DE BRUYĖRE, par Mlle M. F. SḱGoIN, dédiées à M. A. De Lamartine. in-8.

FLEURS DE L'ARRIERE-SAISON (Poésies). In-8. Genève, 1840.

2 fr. 50

FRANCE (1a) CONSTITUTIONNELLE, ou la 'Libert6 reconquise; poème national, par M. BoYARD. In-8. 6 fr.

FRANCE (la) MOURANTE, consultation historique trois personnages. 1829. In-8.

GEOGRAPHIE ANCIENNE DES ÉTATS BARBARESQUES, d'après l'allemand de MANNERT, par MM. Marcus et DuesberG. In-8.

$10 \mathrm{fr}$.

GLAIRES (des), DE LEURS CAUSES, de leurs effets, et des indications à remplir pour les combattre. $8^{\circ}$ édition, par Doussin-Dubreuil. Paris. in-8.

$4 \mathrm{fr}$.

GR AISSINET (M.), ou Qu'est-il donc? Histoire comique satirique et véridique, publiée par DUVAL. $4 \mathrm{v}$. in-12. $10 \mathrm{fr}$.

Ce roman, écrit dans le genre de ceux de Pigault, est un dos plus amusants que nous ayons.

GRAVEUR D'ARGENTERIE (le) de table, par MERCADIER. In-8.

$6 \mathrm{fr}$.

GUIDE DES ARCHITECTES, Vérificateurs, Entrepreneurs et de toutes les personnes qui font bâtir, par L. LEJUSTE. 1 vol. in-40.

$12 \mathrm{fr}$.

GUIDE DE L'INVENTEUR dans les principaux États de l'Europe, ou Précis des lois sur les brevets d'invention, par Ch. Armengaud jeune. In-8. Nour. édit.

$5 \mathrm{fr}$.

GUIDE DES MAIRES (nouveau), ou Manuel des Offi- 
ciers municipaux, dans leurs rapports avec l'ordre administratif ef l'ordre judiciaire, les collèges électoraux, la garde nationale, l'armée, l'administration forestière, l'instruction publique el to clergé; par M. Boy ARD, président à la Cour d'appel d'Or éans, etc. 1 gros vol. in-18 de 612 p. 3 fr 50

GUIDE DU MÉCANICIEN, ou Principes fondamentaux de mécanique expérimentale et théorique, appliqués à la composition et à l'usage des machines, par M. Suzanre, ancien professeur. 2 e édition. 1 vol. in-8 orné d'un grand nombre de planches.

$12 \mathrm{fr}$. in -8.

GUIDE DU PHOTOGRaPHE, par Ce. Chevalier,

GUIDE GÉNÉRAL EN AFFAIRES, ou Recueil des modèles de tous les actes, par J.-B. Noellat. $4^{e}$ édition. rol. in-12.

$4 \mathrm{fr}$.

GUIDE DU PROPRIÉTAIRE ET DE L'ARTISAN, par HANRIOT. in -8.

5 fr.

HARPE HELVÉTIQUE, par CH.-M. Didier. In-8. 1 tr. 50

HISTOIRE AUTHENTIQUE du prisonnier d'Etat connu sous le nom du Masque-de-Fer, extraite des documents trouvés aux archives des affaires étrangères du Royaume; trad.del'anglais de George Agar Ellis. In -8. Э fr.

HISTOIRE D'ANGLETERRE; de DAvid Humz. 20 rol. in-12.

- Plantagenet. 6 rol.

- Tudor. 6 vol.

$18 \mathrm{fr}$.

- Stuart. 8 vol.

$18 \mathrm{fr}$.

HISTOIRE GÉNÉRALE DE LA MUSIQUE ET DE LA DANSE, par Adrien DE LAfage. 2 vol. in-8 et 2 atlas. $1^{\text {re liv. }} 13 \mathrm{fr} .2^{\mathrm{e}}$ liv. $12 \mathrm{fr}$.

$27 \mathrm{fr}$.

HISTOIRE DE LA NATURE ou Synthèse de la création et du perfectionnement des êtres, de Duran, par LAURRIÈRE, in-8.

$1 \mathrm{fr}$.

HISTOIRE DE BAR-SUR-SEINE, par CotTANT. $1^{\text {re }}$ partie, in-8.

HISTOIRE DE LA VILLE DE MULHOUSE, par De la Sablì̀e. 1 vol in-12.

5 fr. 50

HISTOIRE DE LA VILLE D'ORLEANS, de ses édifices, monuments, etc., par VERGNAUD-ROMAGNÉSI. 2 vol. in-12.

HISTOIRE DE LA VILLE DE TOUL et de ses évê. 
ques, suivie d'une Notice sur la cathédrale, ornée de 16 lithographies, par A.-D. ThiÉRY. 2 vol. in-8.

$10 \mathrm{fr}$.

HISTOIRE DES BIBLIOTHEQQUES publique de la Belgique, par NAMUR. 3 vol. in -8 .

$\begin{array}{cr}\text { Tome } 1^{\text {er Bibl. de Bruxelles. }} & 9 \mathrm{fr} . \\ -2^{\circ} \text { Bibl. de Louvain. } & 6 \mathrm{fr} .50 \\ -\quad 5^{\circ} \text { Bibl. de Liëge. } & 6 \mathrm{fr} .50\end{array}$

- DES CAMPAGNES de 1814 et de 1815, par A. DE BEAUCHAMP. 2 vol. in-8.

$12 \mathrm{fr}$.

- DES DOUZE CÉSARS, trad. du latin de Suétone, paI DE LAHARPE. 3 , vol. in-32.

$6 \mathrm{fr} .50$

HISTOIRE DES LÉGIONS POLONAISES EN ITALIE, sous le commandt du général Dombrowski, par LéoNard CHODZKo. 2 vol. in-8.

$17 \mathrm{fr}$.

- DES VANDALES, depuis leur première apparition sur la scène historique jusqu'à la destruction de leur empiro en Afrique; accompagnée de recherches sur le commerce que les Etats barbaresques firent avec l'Etranger dans les six premiers siècles de l'ère chrétienne. $2^{\theta}$ éd. in-8.

7 fr. 50

HISTOIRF، GÉNÉRALE DE POLOGNE, d'après les historiens polonais Naruszewiez, Albertrandy, Czacki, Lelewel, Bandtkie, Niemcewiez, Zielinskis, Kollontay, Ogiaski, Chodzko, Podzeszynski, Mochnacki, et autres écrivains nationaux. 2 vol. iu-8.

$7 \mathrm{ft}$.

HISTOIRE IMPARTIALE DE LA VACGINE, par C.=A. BARREY. In-8.

3 fr. 50

HOMME (l') AUX PORTIONS, ou Conversations philosophiques et politiques, publiées par J.-J. FAzY. 1 vol. in-12.

$3 \mathrm{fr}$.

I BACI DI GIOVANI SECONDO volgarizzati da Cesars L. Bixio. Parigi, 1834, in-12.

$1 \mathrm{fr} .50$

INAUGURATION DU CANAL du duc d'Angoulême : Amiens, le 31 août 1825. In-folio.

1 fr. 50

INFLUENCE (de l') DES ÉRUPTIONS ARTIFICIELLES DANS CERTAINES MALADIES, par JENNER auteur de la découverte de la vaccine. Brochurein-8. 2 fr. 50

INVASION DES ARMEES ETRANGERES dans lo département de l'Aube, en 1814 et 1815, par F.-E. PonGIAT. In-8.

$6 \mathrm{fr}$.

JEANNE HACHETTE, ou le Siège de Beaurais, poème, par madame Fanky Denoix. In-8.

1 fr.

JOURNAL DU PALAIS, présentant la Jurisprudence de la Cour de Cassation et des Cours royales. Nouvelle édition, 
par M. Bourjols. (1791 à 1828.) Paris, 1825 à 1828.42 rol. in-8.

$100 \mathrm{fr}$.

DES VOYAGES, Découvertes e! Navigations modernes, novembre 1818 à déc. 1829.44 vol. in -8 , cart. $176 \mathrm{fr}$.

JOURNALISME (du), ou Il est temps d'en tinir arec le mauraise presse, par D.-J. 1832. In-12.

$50 \mathrm{c}$.

LEÇONS D'ARCHITECTURE, par DURAND. 2 vol. in -4 .

$40 \mathrm{fr}$.

- La partie graphique, ou tome $\tilde{z}^{\theta}$ du mêne ouv. 20 fr. LECONS DE DROIT DE LA NATURE ET DES GENS, par DE FÉLICE. 4 vol. in-12.

$6 \mathrm{fr}$.

LETTERA INTORNO ALL'INTRODUZIONE DEL METODO-WILHEM, nelle Scuole di torino indirizzata, al signor maestro Luici-Felice Rossi, Aal-maestro Adriano DE LAFAGE. In-8.

LETTRES DE JEAN DE MULLER à ses amis MM. De Fonstetten et Gleim. In-8.

$6 \mathrm{fr}$.

- DE MÁDEMOISELLE AISSÉ. In-12. 2 fr. 30 - DE MESDAMES DE VILLARS, DE LA = FAYETTE et DE TENCIN. In-12. 2 fr. 50 - INEDITES de Buffon, J.-J. Rousseau, Voltaire, Piron, de Lalande, Larcher, etc., avec fac simile, publiées par C.-X. Girault. In-8.

- Idem, in-12.

$3 \mathrm{fr}$. in-12, fig.

- SUR LA VALACHIE. 1 vol. in-12.

$3 \mathrm{fr}$.

LIBERTÉS, (des) GARANTIES PAR LA CHARTE, ou do la Magistroture dans ses rapports avec la liberté des cultes, de la presse, etc., par M. Boy ARD. In-8. $6 \mathrm{fr}$.

LOI DU 3 MAI 1841 sur l'Expropriation pour cause d'Utilité publique. Br. in-18.

$30 \mathrm{c}$.

LOIS D'HOWEL-DDA mab Cadeil, Brenin Cymra (fils de Cadell, chef du pays des Kimris), par M. A. Duchatel.s LIER. In-8.

$2 \mathrm{fr}$.

"MACHINES ET INVENTIONS approurées par l'Aca. démie des Scien., par Gallon. 7 vol. in-4. $80 \mathrm{fr}$.

MAGISTRATURE-(de la) dans ses rapports avec la liberté des cultes, par M. Boyand. In-s.

$6 \mathrm{fr}$.

MANIPULATIONS HYDROPLASTIQUES, ou Guide des Doreurs, par M. Roseleur. in 8. $15 \mathrm{fr}$.

MANUEL AIDE-MEMIOIRE DU CONSTRUCTEOR DE 
TRAVAUX PUBLICS ET DE MACHINeS, par EMILE With, 1 vol. in-12, avec fig.

$5 \mathrm{fr}$.

MANUEL (Noureau) COMPLET DES EXPERTS. Iraité des matières civiles, commerciales et administratives donnant lieu à des expertises, $7^{\circ}$ édit., par CH. VASSEROT, avocat à la Cour Impériale de $\mathbf{P a r i s .}$

MANUEL (noureau) COMPLET DES MAIRES, Adjoints, Conseils municipaux, des Préfets, Conseils de préfecture et Conseils-généraux, Juges de paix, Commis.* saires de police, Prêtres, Instituteurs, et des Pères de famille, etc., par M. BoYARD, président à la Cour d'appel d'Orléans. $3^{\mathrm{e}}$ édition, 2 vol. in-8.

$12 \mathrm{fr}$.

MANUEL DE L'ECARTE, contenani des notions gécírales sur teo $2^{e}$ édition. Bordeaux in-18.

î.

MANUEL DE L'OCULISTE, ou Dictionnaire ophthalmologique, par DE WENzEL. 2 vol. in-8, 24 planches. 12 fr.

- DES ARBITRES, ou Traité des principales connaissances nécessaires pour instruire et juger les affaires soumises aux décisions arbitrales, soit en matières civiles ou commerciales; contenant les principes, les lois nouvelles, les décisions intervenues depuis la publication de nos Codes, et les formules qui concernent l'arbitrage, etc., par M. CH., ancien jurisconsulte. Nouvelle édition.

$\delta$ fr.

- DFS BAINS DE MER, leurs avantages et leurs inconpénients, par M. BLot. 1 vol. in-18.

$2 \mathrm{fr}$.

- BIBLIOGRAPHIE UNIVERSELLE, par MM. F. Denis, Pincon et de Martonne, 1 vol. gr. in-8 à 5 col. papier collé pour recevoir des notes.

$25 \mathrm{fr}$.

- DES CANDIDATS à l'emploi de Vérificateurs des poiủs et mesures, par P. RAvor. $2^{e}$ édition, in -8 . $5 \mathrm{fr}$. - DES JUSTICES DE PAIX, ou Traité des fonctions é des attributions des Juges de Paix, des Greffiers et Huissiers attacbés à leur tribunal, avec des formules et modèles do tous les actes qui dépendent de leur ministère, etć, par $\mathbf{M}$. LEvassaun, ancien jurisconsulte. Nouvelle édition, entièrement refondue, par M. BIBET. 1 gros volume in-8. 1839.

$6 \mathrm{fr}$.

- Idem, en 1 vol. in-18.

3 fr. 50

- DE LAMATEUR D'HUITRES, par Al. Martin. 1 rol. in-18.

$1 \mathrm{fr}$.

MANUEL DES NÉGOCIANTS, ou le Code commercial et maitime, commenté et démontré par principes, par P.-B. BOUCHER. 2 vol. in-8. 
MANUEl DES NOURRICES, par Mme El. Celnart. In-18.

- DU BOTTIER, par A ModRey. In-12. 1 fr. 50

- DU CAPITALISTE, par M. BoNNET. 1 pol. in-8. 16e édition.

$6 \mathrm{fr}$.

- DU FABRICANT DE ROUENNERIES, comprenant tout ce qui a rapport à la fabrication, par un Fabricant. 1 rol. in-18.

2 fr. 50

MANUEL DU NÉGOCIANT, dans ses rapports avec la douane par M. BAUZON-MAGNIER. In-12. $4 \mathrm{fr}$.

MANUEL DES SOCIÉTÉS DE SECOURS MUTUELS, in 12 , br.

$50 \mathrm{c}$.

- DU SYSTÈME MÉTRIQUE, ou Livre de Réduction de toutes les mesures et monnaies des quatre parties du monde, par P.-L. LIONET. 1 vol. in-8.

$7 \mathrm{fr}$.

MANUEL DU TISSEUR, contenant les Armures et les Montages usités pour la Fabrication des divers Tịsus, par Lions. In-8.

$1 \mathrm{fr}$.

MANUEL .DU TOURNEUR, ourrage dans lequel on enseigne aux amateurs la manière d'exécuter tout ceque l'art peut produire d'utile et d'agréable, par M. HaMELIN-BERGERON. 2 vol in-4, avec Atlas et le Supplément. 39 fr. - MÉTRIQUE DU MARCHAND DE BOIS, par M. Tremblay. 1 rol. in-12. 1840. 1 fr. 50

MATERIAUX POUR L'HISTOIRE DE GENĖVE, recueill is et publies par J.-A. Galiffe. tome 1 , in-8. 6 fr. MÉDECINE POMESTIQUE, ou Traité complet des moyens de se conserver en santé, et de guérir les maladies par le régime et les remèdes simples, par BucHAN; traduii par Duplanil. 5 vol. in-8.

$20 \mathrm{fr}$.

MÉDITA TIONS L YRIQUES, par J.-J. GALLOIS. In-8.

MÉLANGES DE POÉSIE ET DE LITTÉRATURE, par Florian. 3 vol. in- 18.

3 fr. 50

MEMENTO DES ARCHITE CTES ET INGÉNIEURS, TOISEURS FT VERIFICATEURS et de toutes les personnęs qui font bâtir, 7 vol. in- 8 ornés de pl. $\quad 60$ fr.

MÉMOIRE SUR LA CONSTRUCTION DES INSTRUMENTS à Cordes et à Archet, par Félix Sa vart. In-8. 3 fr.

MÉMOIRES DU COMTE DE GRAMMONT, par HAKILTON. 2 vol. in-32.

3 fr.

MÉMOIRES RÉCRÉATIFS, SCIENTIFIQUES ET ANECDOTIQUES, du physicien-aéronaute ROBERTSON. a vol. in-8, fig.

12 fr. 
MÉMOIRES SUR LA GUERRE DE 1809 EN ALLEMAGNE, a vec les opérations particulières des corps d'Italie. de Pologne, de Saxe, de Naples et de Walcheren, par le général Pecet, d'après son journal forl détaillé de la campagne d'Allemagne, ses reconnaissances eh ses divers travaux; la correspondance de Napoléon avec le major-général, les maréchaux, etc. 4 vol. in-8.

$28 \mathrm{fr}$.

MÉMOIRE SUR LES OPÉRATIONS de l'avant-garde da $8^{\circ}$ Corps de la Grande Armée, formé de troupes polonaises en 1S13. In-8.

MÉMOIRE SUR UES SCULPTURES ANTIQUES, par VEKGNAOD. In-S.

$1 \mathrm{fr}$.

MÉNESTREL (le), poème en deux chants, par JAMES BeATtie; traduit de l'anglais, avec le texte en regard, par M. LOUET. 28 édition, in-18.

$3 \mathrm{fr}$.

MENUISERIE DESCRIPTIVE, nouveau Vignole de: menuisiers, utile aux ouvriers, maîtres et entrepreneurs, pay Codlor, 2 vol. in-4, dont un de planches.

$20 \mathrm{fr}$.

MINISTRE DE WAKEFIELD, traduit en français par M.Aignan, de l'Académie française. Nouvelle édition; 1841 . 1 rol. in -12 , fig.

1 fr. 50

MONITEUR DE L'EXPOSITION de 1839 , ou Archives des produits de l'inảustrie. In-8.

5 fr.

MORALE DE L'ÉVANGILE, comparéeà la morale des philcsophes anciens et modernes, par madame E. Celrart. In-8.

MULTIPLICATEURS DES INTÉRÊTS SIMPLES, établis sur les taux de 3 , 4 el 5 pour cent, etc., par MoREA U, $1^{\text {re }}$ partie. 1 vol. in $-8^{\circ}$ obl.

3 fr. 50

NÉCESSITÉ (de la) ET DE L'EXPÉRIENCE, consiłérées commécritérium de la vérité, $\mathrm{par} \mathrm{G}_{0}-\mathrm{M}^{\star \star \star}$. in-8. $7 \mathrm{f}$. 50

NOSOGRAPHIE GÉNÉRALE ÉLÉMENTAIRE, ou Description et Traitement rationnel de toutes les maladies; par M. Seigneur Gens, docteur de la Faculté de Paris. Nourelle édition, 4 vol. in-8.

$20 \mathrm{fr}$.

NOTES SUR LES PRISONS DE LA SUISSE, et sur quelques-unes du continent de l'Europe; moyen de les améliorer, par M. Fr. CuninghaM; suivies de la description des prisons améliorées de Gand, Philadelphie, Ilchestes et Millbank, par M. BUXToN. In-8.

4 fr. 50

NOTICE HISTORIQUE sur la vilie de Toul, ses antiquités et ses célébrités, par G.-L. Bataille. In-8. \& fr. 
NOTICE HISTORIQUE sur les magnifiques tapisseries des Gobelins, par LACORDAIRE, in-80.

SUR LA PROJECTION DES CARTES GEOGRAPHIQUES, par E-A. LEYMONNERYE. In-18, figures.

$1 \mathrm{fr} .50$

- SUR L'OEUVRE de François Girardon, de Trojes, sculpteur, a vec un précis sur sa vie. In-8.

$1 \mathrm{fr} .50$

NOTIONS SYNTHETIQUES, historiques et physiologiques de philosophie naturelle, par M. GEOFFroY-ST-HILAIRE. In-8.

NOVELLE ITALIANE DI GIOVANNI LA GECILIA. In-8.

$4 \mathrm{fr}$.

NOUVELLE MÉTHODE DE TENUE DES LIVRES, par Nicol. Br. in -8 .

$75 \mathrm{c}$.

* OEUVRES CHOISIES de l'abbé Prévost, avec fig. 39 vol. in -8 , reliés.

$100 \mathrm{fr}$.

OBSERVATIONS SUR LES PERTES DE SANG des femmes en couche et sur les moyens de les guérir, par M. LERoUx. $2^{\mathrm{e}}$ édition. In-8.

4 fr. 50

OBSERVATIONS SUR UN ARTICLE de la Revue Eneyclopédique relatif à la traduction du Talmud de Babylone, et à la théorie du judaïsme, par l'abbé CHIARINI. In-8. 2 fr.

OEUVRES COMPLÉTES DE CHAMFORT, revueillie et publiées par P.-A. A UGUIs. 5 vol. in-8. $15 \mathrm{fr}$. OEUVRES DE BALLANCHE, de l'Académie de Lyon. 4 rol. in-18.

$15 \mathrm{fr}$.

OEUVRES DE BOILEAU, nouvelle édition, accompagaées de Notes failes sur Boileau par les commentateurs ou littérateurs'les plus distingués, par M. J. Planche, professeur de rhétorique au collége royal de Bourbon, et $\mathbf{M}$. NOEL, inspecteur général del'Université. In-12. 1 fr. 50

- DE SERVAN, nourelle édition, arec une notice, par X. De Portets. 5 vol. in-8.

$18 \mathrm{fr}$.

OEUVRES DE VOLTAIRE, avec Préfaces, Averlissements, Noles, etc., par M. Beuchot, 1. 71 et 72. TABLE ALPHABÉTIQUE ET ANALYTIQUE DES MATIÈRES, par Miger. 2 vol. in-8. $24 \mathrm{fr}$. Idem. papier vélin. Wom, grand papier jésus. $36 \mathrm{fr}$. $48 \mathrm{fr}$. OEUVRES D'ÉVARISTE PARNY. 5 rol. in -18 . 12 fr. 50

OEUVRES DIVERSES DE LAHARPE, de l'Áádèmie française, 16 rol. in-8. $64 \mathrm{fr}$. 
OEUVRES DIVERSES. Économie politique; Instruction publi que; Haras et Remontes, par C.-J.-A. MATHIEU DB DOMBASLE. In-8.

$8 \mathrm{fr}$.

- DRAMATIQUES DE $\mathbf{N}$. DESTOUCHES. Nourelle odition. Paris. 6 vol. in -8

$24 \mathrm{fr}$.

- POETIQUES DE KRASICKI. 1 seul rol. in-8, à 2 col.grand papier vélin.

$25 \mathrm{fr}$.

OPUSCULES FINANCIERS sur l'effet des privilèges, des emprunts publics et des conversions sur le crédit de l'industrie en France, par J.-.J. FAzY. 1 vol. in-8.

5 fr.

ORDONNANCE SUR L'EXERGICE ET LES MANOEUVRES D'INFANTERIE, du 4 mars 1831. (Ecole da soldat et de peloton). 1 vol. in-18, orné de fig.

$75 \mathrm{c}$.

ORGUE (I') DE SAINT-DENIS, par LAFAGE. In-8. 2 fr.

OUVRIER (I') MECANICIEN, Guide de mécanique pratique, précédé de notions élémentaires d'arithmétique décimale, d'algèbre et de géométrie, par Ch. ARmengadD jeune. $5^{\theta}$ édition, in-12.

$4 \mathrm{fr}$.

PARFAIT CHARRON - CARROSSIER, ou Traité complet des Ouvrages faits en C'harronnage et Ferrure, par L. BERTHAUX. In-8.

$10 \mathrm{fr}$.

- Le Parfait Charron, seul.

5 fr.

- Le Parfait Carrossier, seul.

$5 \mathrm{fr}$.

PARFAIT SERRURIER, ou Traité des ouvrages faits en fer, par Lodis BerthadX, 1 vol. in -8, cartonné. 9 fr. PASSE (DU), DU PRÉSENT ET DE L'AYENIR do l'Organisation municipale de la France, par E. CHAMPAGNAC, tome 1 er, in -8.

$4 \mathrm{fr}$.

PETIT (le) BAREMME DES CAISSES 'D'ÉPARGNE, ou Mélhode simple et facile pour calculer les intérêts depuis 1 jusqu'à 40 ans, par Van-Tenac. In-32.

PETIT MANUEL. DU NEGOCIANT D'EAU-DEVIE, par Ravon el MaLepeyre. In-18.

75 e.

PETIT PAMPHLET sur quelques tableaux du salon de 1855 , par A.-D. VeRGNAUD. In-8.

$30 \mathrm{c}$.

PHILOSOPHIE ANTI-NEWTONIENNE, ou Essai sur une nourelle physique de l'univers, par J. BaUtés. Paris, 1833 , 2 livraisons in -8.

$3 \mathrm{fr}$.

PHOTOGRAPHIQUe (Album), par M. BlanqdartEvrakd. Livraisons 1 à 12 , contenant chacune 3 planches. Ouvrage complet.

72 fr.

PHOTOGRAPHIE SUR PLAQUES MÉTALLIQUES, par M. le baron Gros, $2^{\circ}$ édition, in-8. fig.

$3 \mathrm{fl}$. 
PHOTOGRAPHIE SUR PAPIER, par M. BLANQUART -EVRARD. Brochure in-8.

POESIES DE CHARLES FROMENT. 2 vol. in-18. $7 \mathrm{fr}$.

POÉTES (les) FRANCAIS depuis le $\mathrm{XI1}^{\circ}$ siècle jusqu'à Malherbe, avec une Notice historique et littéraire sur chaque poète. Paris, 1824, 6 vol. in-8.

$48 \mathrm{fr}$.

POEZYE ADAMA MICKIEWICZA, 4 rol. In-12. Prix de chacun.

$5 \mathrm{fr}$.

POLITIQJJE POPULAIRE, ou Manuel des droits et des devoirs du citoyen, In-18 carré.

PRÉCIS DE L'HISTOIRE DES TRIBUNAUX SECRETS DANS LE NORD DE L'AILEMAGNE, par A. Loeve Veimars. 1 ro!. in-18.

1 fr. 25

- HISTORIQUE SUR LES RÉVOLUTIONS DES ROYAUMES DE NAPLES ET DU PIÉMONT, en 1820 et 1821 , suivi de documents authentiques sur ces èrènements, par M. le comle D.... 22e édition. In-8. 4 fr. 50

PROJET D'UN NOUVEAU SYSTĖME BIBLIOGRAPHIQUE des Connaissances hugnaines; par NAMUk. In-8. $4 \mathrm{fr}$.

QUELQUES RÉFLEXIONS sur la Législation commerciale, par A.-J. Menot. Paris, 1823. In-8. 2 fr. 50

QUESTION DE LORIENT sous ses rapports généraur et particuliers, par M. De Pradt. In-8.

QUESTION DES ENTREPOTS ET PORTS FRANCS, contenant onz lettres publiées dans le journal le Commerce de Dunkerque et du Nord, par M. Battier. Grand in-8. $3 \mathrm{fr}$.

RAPPORT FAIT A LA CHAMBRE des Representant et au Sénat, par le Ministre des affaires étrangères, sur l'état des nćgociations en 1851. Bruxelles, in-8. $6 \mathrm{fr}$.

RAPPORTS DES MONNAIES, POIDS ET MESURES des priocipaux Etats de l'Europe (ce tarif est collé sur bois). $3 \mathrm{fr}$.

RAYONS les) DU MATIN, poésies par Elie Sa UVAgE. In-18.

2 fr. 50

RECHERCHES ANATOMIQUES, Physiologiques, $\mathbf{P}_{2-}$ thologiques et Séméiologiques, sur les glandes labiales, $p a r$ A.-A. Sebastian. In-

2 fr. 50

- SUR L'ANATOMIE et les Métamorphoses de différentes espèces d'insectes; ouvrage posthume, de PIER RE Gyonnet, publié par M. W. DeBAAN; accompagnées do 54 planches. 1 vol in- 1.

$40 \mathrm{fr}$.

- HISTORIQUES SUR LA VILLE DE SALINS, par M. BECHET. 2 vol. in- 12. $5 \mathrm{fr}$. 
RECHERCHES (Nouvelles) sur les mouvements du camphre et de quelques autres corps placés à la surface de l'eau, par MM. Joly et Boisgiraud aîné. In-8

1 fr. 50

- SUR LE RÉGIME ANCIEN DES EAUX DE LA SEINE sur le territoire de Troyes, par Deniel. Br. in $-8^{\circ}$.

3 fr. 50

- SUR LE SYSTÈME LYMPHATICO-CHYLIFẼRE par le docteur LIPPI; traduit de l'italien par JULIA DE FONTENELLE. In-8.

$75 \mathrm{c}$.

- SUR La TÉlÉgraPHe ÉlECTRIQUE, par Gloesener. In-8, avec figurès.

3 fr. 50

RECUEIL DE MÉMOIRES SUR LA PHOTOGRAPHIE, par Ch. Cinevalier. Grand in-8. 3 fr. - E'T PARALLELES D'ARCHITECTŨR, par M. Durand. Grand in-fol.

$180 \mathrm{fr}$.

DE RECETTES ET DE PRÉPARATIONS CHIMIQUeS, d'objets d'un usage journalier, brochure in-18.

$75 \mathrm{c}$.

- GÉNÉRAL ET RAISONNÉ DE LA JURISPRUDENCE et des attributions des justices de paix, en toutes matières, civiles, criminelles, de police, de commerce, d'octroi, de douanes, de brevets d'invention, contentieuses et non contentieuses, etc., par M. Biret. $4^{\theta}$ éd. in-8. 2 vol. $14 \mathrm{fr}$. REFORME (de la) ANGLAISE et de ses suites probables, par M. De Phadt. In-8.

3 fr.

REGLES DE POINTAGE à bord des vaisseaux, par MontgéRY. In-8.

RÉgNiCide et Régicide, par M. De Pradt. In-8.

$75 \mathrm{c}$.

RELATION (nouvelle) DE LA BATAILLE DE FRIEDLAND (14 juin 1807), par M. DEROdE. In-8. 2 fr. 25 -Idem, Papier vélin.

$3 \mathrm{fr}$.

RELATION DU VOYAGE AU POLE SUD ET DANS L'OCEANIE, sur les corvettes l'A strolabe et la Zélée, exécuté par ordre du Roi pendant les années $183 i, 1838,1839$ ef 1840 , sous le commandement de M. J. DuMONT-D'URVILLE, capitaine de vaisseau. 10 vol. in-8, a vec cartes. $30 \mathrm{fr}$. RELATIONS DE VOYAGES D'AUCHER-ELLY EN ORIENT, de 1830 à 1838 , revues et annotées par $M$. lo comte JADBERT. 2 vol. in-8, a vec carte.

$12 \mathrm{fr}$.

RELIGION (de la), DU CLERGÉ ET DES JÉSUITES, par un Magistrat. 1844 . In-8.

1 fr. 23. 
REPPERTOIRE ADMINISTRATIF DES PARQUETS, par L.-G. FaURe. 2 vol. in-8.

$15 \mathrm{fr}$.

- (Supplément au) par FaURE, 1 vol in-8 (1855). 7 f. 50

- (Noureau) DE LA JURISPRUDENCE et de la Science du Notariat, par J.-J.-S. Serieys. In-8. 7 fr.

RÉPUBLIQUE (la) PARTHÉNOPÉENNE, épisode de l'histoire de la répnblique française, par Jean La CÉcIlia. Traduit de l'italien par THibaud. In-S.

7 fr. 50

RÉSERVE (De la) LÉGALE en Matière de Succession, et de ses conséquences, par J.-B. KuHLMaNN. In-8. 1 fr. 50

REVISION IMMEDIATE DE LA CONSTITUTION avec la Sanction du Peuple, par Boyard. Br. in-8. $40 \mathrm{c}$.

REVOLUTIONS DE CONSTANTINOPLE en 1807 ef 1808 , précédées d'observations sur l'empire ottoman, par A. De Jucheread de Saint-Denis. 2 vol. in-8. 9 fr.

- DE JUILLET 1830. Caractère légal et politique du nouvel établissement fondé par la Charte constitutionnelle. 1833. In-8.

$1 \mathrm{fr} .50$

RODRIGUE ET EUDOXIE, dialogue en vers et en prose, par A.-F. Gérard. In-12.

$1 \mathrm{fr}$.

ROMAN COMIQUE, par ScarroN, nouvelle édition revue el augmentée. 4 vol. in-12.

RUSSIE (la) E T L'EMPIRE OTTOMAN tels qu'ils sont et tels qu'ils devraient être, par N.-J.-B. Boyarn. 1 vol. in 8.

5 fr.

SCULPTEUR PARISIEN (Album du), par GoIlMaRd. 1 vol. in-4, cart.

$12 \mathrm{fr}$.

SÉCRETISME (le) ANIMAL, nouveile doctrine fondée sur la philosophie médicale, par A.Christophe.In-8. 3 fr.

SIDEROTECHNIE (la), ou l'art de traiter les minerais de fer poue en obtenir de la fonte, du fer ou del'acier, par J.-H. Hassenfratz. 4 beaux vol. in- $4^{0}$ bien rel 60 , fr.

SIÉCLE (le), Revue critique de la littérature, des Sciences et des. Arts. 2 vol. in-8.

$20 \mathrm{fr}$.

SIGNES DE CORRECTION, par FreY. 1 flle. $75 \mathrm{c}$.

SITES PITTORESQUES DU DAUPHINÉ, dessinés d'après nature et lithog., par DaGNAN. In-fo . 40 vues. $50 \mathrm{fr}$.

- Chaque vue séparément.

$2 \mathrm{fr}$.

SOIREES DE MADRID, ou Recueil de nouvelles historiettes, etc., par $M^{\text {me }} A M^{\prime}$ DÉ E DE $^{\star \star \star \star} .4$ vol, in-12.10 fr.

SOURCE ( La ) DE LA VIE, ou Choix d'Idées, Axiomes, Sentences, Maximes, etc., contenus dans lo Talmud, trad. par SAMSON LÉvy. 2 parties, in-12. 4 fr. 
SOUVENIRS DE MADAME DE CAYLUS, suivis de quelques-unes de ses lettres. Nouv. édit. in-12. $2 \mathrm{fr} .50$ STATISTIQUE DE LA SUISSE, par M. Picot, de Genève. 1 gros vol. in-1 2 de plus de 600 pages.

$7 \mathrm{fr}$. SUÈDE (la) SOUS CHARLES XIV JEAN, par FR. SCHMIDT. In-S.

SUITE AU MÉMORIAL DE SAINTE-HÉLENE, Orné du portrait de M. Las-Case. 1 vol.in-8.

* SUITE DU RÉPERTOIRE DU THEATRE FRAN ÇAIS, par Lepeintre. Paris, V ${ }^{\circ}$ Dabo. 81 vol. in-18. 60 Pr.

TABLE ALPHABETIQUE ET CHRONOLOGIQUE des instructions et circulaires émanées du Ministère do la justice, depuis 1795 jusqu'au 1 er janvier 1837, par M. MASSABIAU. 1 vol. in-4.

3 fr. 50

TABLEAU DES PRINCIPAUX ÉVENEMENTS QUI SE SONT PASSÉS A REIMS, depuis Jules-César jusqu'à Louis XVI inclusirement, par M. Camus-Daras. 2o édition, revue et augmentée. 1 rol. in-8.

$10 \mathrm{fr}$.

TABLETTES BRUXELLOISES, ou Usages, mœura et coutumes de Bruxelles, par MM. ImBert et Bellet. In18.

TARIF DU CUBAGE DES BOIS CARRÉS, par Francon. Bro in-8o.

2 fr. 50

TARIF (Nouveau) DES PRIX COMPARATIFS des ânciennes et nourelles mesures, suivi d'un abrégé de géométrie graphique, par Roussead. In-12.

2 fr. 50

TLiEORIE DES SIGNES, ou Introduction à l'élude des langues, par l'abbé SiCARD. 2 vol. in.8.

THEORIE DU JUDAISME appliquée à la réforme des Israélites de toutes les parties de l'Europe, par l'abbé L.-A. CHiarimi. 2 vol. in -8 .

$10 \mathrm{fr}$.

TOILETTE (La) DE FLORE, par J. P. In-8. 6 fr. TOURNEUR (Suppléinent à tous les ourrages sur l'art du). Orné de planches. In-4.

TRAITE COMPLET DE LA FILATURE DU CHANVRE ET DU LIN, par MM. Coquelin et DECoster 1 gros rol. avec un bel Atlas in-folio, renfermant 37 planches gravéesarec beaucoup de soin. Paris, 1846.

TRAITE DU CHAUFFAGE AU GAZ, par Ch. HUGUENY. Br. in-8, prix

$1 \mathrm{fr} .50$

TRAITÉ DE CHIMIE APPLIQUÉE AUX ARTS ET MÉTIERS, et principalement à la fabrication des acides sulfurique, nitrique, muriatique ou hydro-chlorique; de la 
soude, de l'ammoniac, du cinabre, minium, céruse, alun, couperose, vitriol, verdet, bleu de cobalt, bleu de Prusse, jaune de chrôme, jaune de Naples, stéarine et autres produits chimiques; des eaux minérales, de l'éther, du sublimé, du kermès, de la morphine, de la quinine, et autres préparations pharmaceutiques; du sel, de l'acier, du fer-blanc, de la poudre fulminante, etc., etc., par M.J.-J. Guilloud, professeur de chimie et de physique; arec planches, représentant près de 60 figures. 2 forts vol. in-12.

$10 \mathrm{fr}$.

TRAITÉ DE DORURE ET ARGENTURE GALVANIQUES appliquées à l'hoslogerie, in- $8^{\circ}$, par OLIVIER MATHEY.

1 fr 25

TRAITE DE LA COMPTABILITÉ DU MENUISIER, applicable à tous les états de la bàtisse, par D. Clousier. 1 vol. in-8.

2 fr. 50

TRAITÉ DE LA COUPE DES PIERRES, ou Méthode facile et abrégée pour se perfectionner dans cette

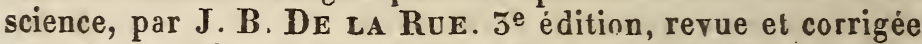
par M. RaMÉE, architecte. 1 vol. in-8 de texte, arec un atlas de 98 planches in-fol.

$20 \mathrm{fr}$.

TRAITÉ DES ÉCHAFA UDAGES, ou Choix des meilleurs modèles de charpentes, par J. CH. KRAFFt. 1 vol. in-fol. relié, renfermant $51 \mathrm{pl}$. très-bien gravées. $25 \mathrm{fr}$.

TRAITÉ DES MANIPULATIONS ÉLECTRO CHIMIQUES, appliquées aux arts et à l'industrie, par $\mathbf{M}$. Brandely, ingénieur civil, in- $8^{0}$, orné de $6 \mathrm{pl}$. $5 \mathrm{fr}$.

TRAITE DE LA MORT CIVILE en France, parA.-T. DESQUIRON. In-8.

$7 \mathrm{fr}$.

TRAITE DES MOYENS DE RECONNAITRE LES FALSIFICATIONS des Drogues simples el composées, et d'en constater le degré de pureté, par BUSSY et BOUTBonCharlard. In-8.

$7 \mathrm{fr}$.

- DE LA POUDRE la plus convenable aux armes à piston, par VERGNAUD aîné. In-18. $75 \mathrm{c}$.

- DE PIIYSIQUE APPLIQUÉE A UX ARTSET MÉTIERS, et principalement à la construction des fourneaur, des calorifères à air et à vapeur, des machines à vapeur, des pompes; à l'art du fumiste, de l'opticien, du distillateur; aux sécheries, artillerie à vapeur, éclairage, bélier et presses hydrauliques, aréomètres, lampes à niveav constant, etc., par J.-J. GUILLodd, professeur de chimie et de physique; arec pl. représentant $160 \mathrm{fig}$. 1 fort vol. in 18 . $3 \mathrm{fr} .30$ 
TRAITÉ D'ÉQUITATION sur des bases géométriques, contenant 74 figures, par A.-C.-M. Parisot. In-8. 10 fr.

TRAITÉ NES ABSENTS, contenant des Lois, Arrêté, Décrets, etc., par M. TalaNdier. In-8.

$7 \mathrm{fr}$.

- DES PARAFOUdRes ET DES PARAgRÈLES, on cordes de paille, $3^{\theta}$ suppl., par Lapostole. In-8. 1 fr. 50

- ÉlÉMENTAIRE de la FILATURE du COTON, par M. OGER, directeur de filature, et SALAdiN. In-8 ef Atlas.

$18 \mathrm{fr}$.

- ELÉMENTAIRE DU PARAGE ET DU TISSAGE MÉCANIQUE DU COTON, par L. BEDEL el E. BOURCART. In-8, fig.

7 fr. 50 .

- PRATIQUe DE CHIMIE appliquée aux arts el manufactures, à l'hygiène et à l'économie domestique, par GraY. Traduit par Richard. 3 vol. in-8 et Atlas. 30 fr.

TRAITE DE LA FABRICATION DES TISSUS, par Falcot, 2 vol. in-4 de texte, plus 1 Allas orné de beaucoup de planches.

$50 \mathrm{fr}$.

TRAITÉ DE GÉODÉSIE PRATIQUE, par GoriN. 1 vol. in. 8 .

2 fr. 50

TRAITE D'ASTRONOMIE, par LALANDE. 4 vol. in- $4^{\circ}$ reliés.

$50 \mathrm{fr}$.

- SUR LA NOUVELLE DÉCOUVERTE DU LEVIER VOI.UTE, dit LEVIER-VINET. In-18. 1 fr. 50 TRAITE DE VOTATION, ou Machines à Voter, inventées par J. RaYMondr. Grand in-8 avec fig. $1 \mathrm{fr}$. 50

TRANSMISSIONS A GRANDES VITESSES. - $\boldsymbol{P}_{a-}$ liers-graisseurs de M. De Coster, par Benoit-Duportall. In-8.

$73 \mathrm{c}$.

TROIS RÉGNES de l'Histoire d'Angleterre, par M. Sadquairé SoUligné. 2 vol. in-8.

$10 \mathrm{fr}$.

UNE ANNEE, ou la France depuis le 2? juillel 1830 , jusqu'au 27 juillet 1831, par M. DE JA1LLY. In-8. 7 fr.

VACCINE (de la) et ses heureux' résultats, par MM. Bronet, Doussin-Dubreuil et Charmont. In-8. 4 fr.

VÉRITABLE (le) ESPRIT de J.-J. Rousseau, par l'abbé Sabatiez de Castres. 3 vol.in-8.

$15 \mathrm{fr}$.

VICTOIRES, Conquêtes, Désastres, Revers et Guerrez broise Burély, par RABAUd-SAINT-ETIENNE.In-18.1 fr.73

VIGNOLE DU CHARPENTIER. $1^{\mathrm{r} \theta}$ partie, ART DU TRAIT, contenant l'application de cet art aux principales constructions en usage dans le bâtimenl, par M. Michel, 
maître charpentier, et M. BouterEAU, professeur de géométrie appliquée aux arts. 1 vol. avec atlas renfermant 72 planches gravées sur acier. Prix :

$20 \mathrm{fr}$.

VIRGINIE, ou l'Enthousiasme de l'Honneur, tiré de l'hig-

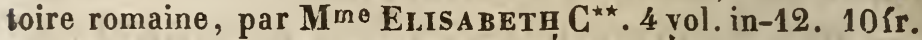

VISITE DE MADAME DE SEVIGNÉ, à l'occasion de la révocation de l'édit de Nantes, ou le Rubis du Père Lachaise. In-8.

VOCABULAIRE DU BERRY et de quelques cantons roisins, par un amateur du vieux langage. 1 vol. in-\$. $3 \mathrm{fr}$.

VOYAGE DE DÉCOUVERTE AUTOUR DU MONDE, ot à la recherche de La Pérouse, par M. J. DuMont D'URville, capitaine de vaisseau, exécuté sous son commandement et par ordre du gouvernement, sur la corvettel'A strolabe, pendant les années 1826, 1827, 1828 et 1829. Histoire du Voyage, 5 gros vol. in-8, arec des vignettes en bois, dessinées par MM. DE SAIrson et Tony JohanNot, gravées par Porret, accompagnées d'un Atlas contenant 20 planches ou cartes grand in-fol.

$60 \mathrm{fr}$.

Cet important ouvrage, totalement terminé, qui a été exécuté par le gouvernement sous le commandement de $M$. Dumons d'Urville et rédigé par lui, n'a rien de oommun avec le. voyage pittoresque publié sous sa direction.

VOYAGE HISTORIQUE dans le département de l'A ube, en vers. In-8.

1 fr. 50

- MÉDICAL AUTOUR DU MONDE, exécuté sur la corvette du roi la Coquille, commandée par le capitaine Duperrey, pendant les années $1822,1823,1824$ et 1825 , suiv: d'un Mémoire sur les Races humaines répandues dans l'Océanie. la Malaisie et l'A ustralie, par M. LEssor. In-8. $4 \mathrm{fr} .5 \mathrm{C}$

VOYAGE EN AISACE, par RouvRoIs. 1 vol. gr in-80 illustré.

$4 \mathrm{fr} .51$

- AUX PRAIRIES OSAGES, Louisiane et Missouri \$839-40, par VICTOR TIXIER. In-8.

$3 \mathrm{fr}$

* - IMAGINAIRES, Songes, Visions et Romans cabalis tiques, ornés de figures. 39 vol. in-8, rel.

$100 \mathrm{fs}$ 


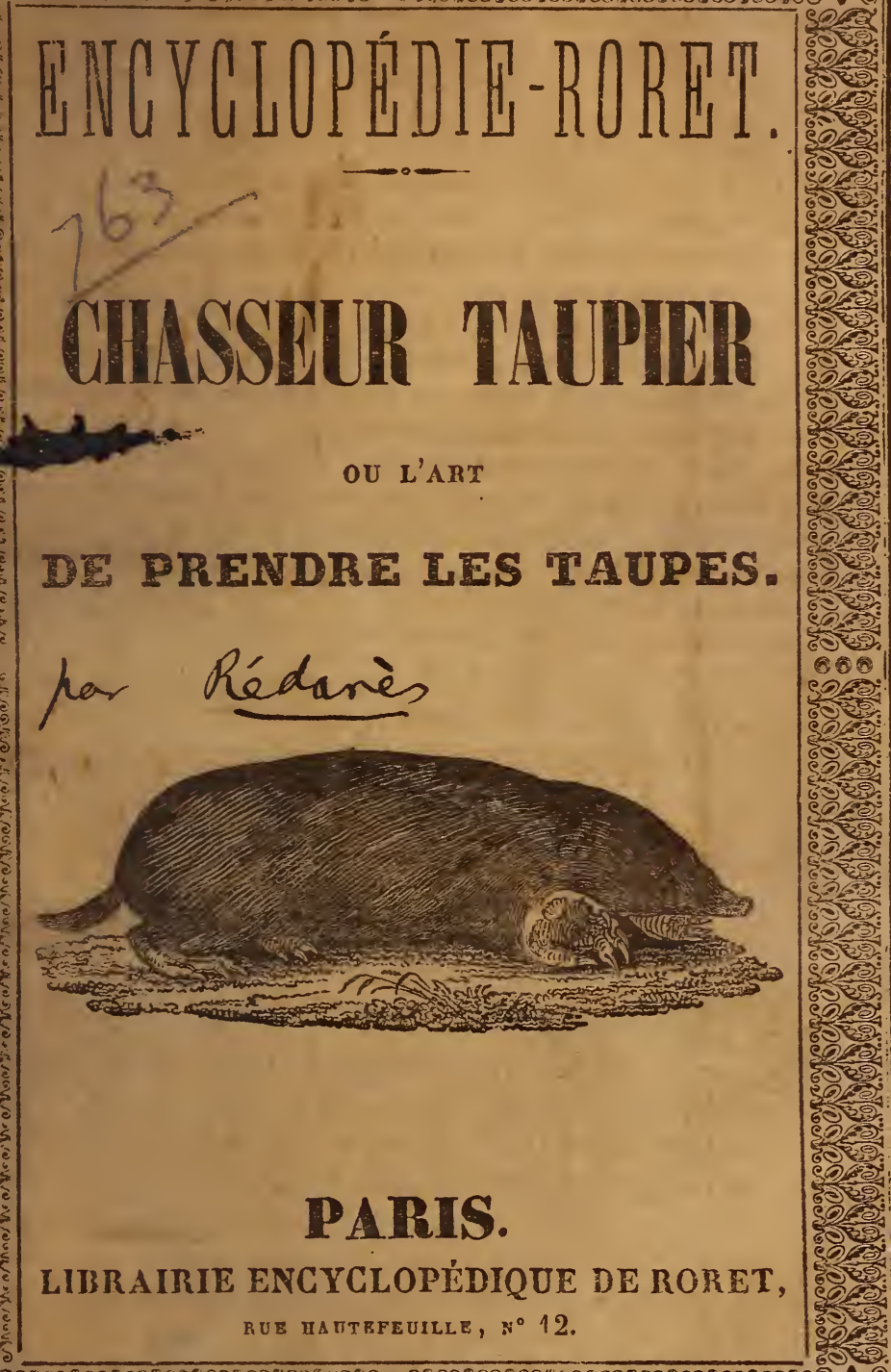

है 
valeurs, rendit 2,600 fr. de meubles; mais là que, peu de jours après, une facture $37,750 \mathrm{fr}$. lui est envoyée. M. de Lizardi it consentir à payer des curiosités, objets de i caprice, beaucoup plus qu'elles ne valent, is il ne peut accepter les exagérations les is extraordinaires. Il demanda que cette face fût l'objet d'un réglement. Alors on orgasa contre lui un tuut petit scandale, on fit ce ocès. On a obtenu la permiśsion de faire une isie conservatoire chez Mlle Delamarre, que jser n'avait pas vue au moment où le marché tait fait, qu'il ne connaissait que comme voiae. C'était habile. On espérait porter la guerre itre Mlle Delamarre et MI. de Lizardi; aujourhui nous venons demander la main-levée de tte saisie.

Mlle Delamarre a-t-elle acheté chez vous? La mnaissez-vous? Non; la facture est au nom de . de Lizardi. Lui auriez-vous vendu même? ertainement non ; vous auriez dit: Sa solvabité ne vaut rien. Mlle Delamarre n'a rien à dénêler avec vous; on lui a fait un cadeau, c€ adeau est chez elle; il n'est pas chez M. de Liardi, qui demeure ailleurs. Quant à sa solvasilité, il l'établit en représentant une police l'assurance qui constate que le mobilier de hlle Delamare, non compris les présens de M. le Lizardi, est assuré pour la valeur de deux cent quarante-trois mille francs.

Mais ce n’est pas là le point capital du procès.

Ce que nous voulons, c'est que les prix fabuleux dont M. Moser réclame le paiement soient appréciés par un expert. M. de Lizardi n’a pas débattu ces prix, les sommes qu'il a payées ne cont nas une reconnaissance de la vente aux 


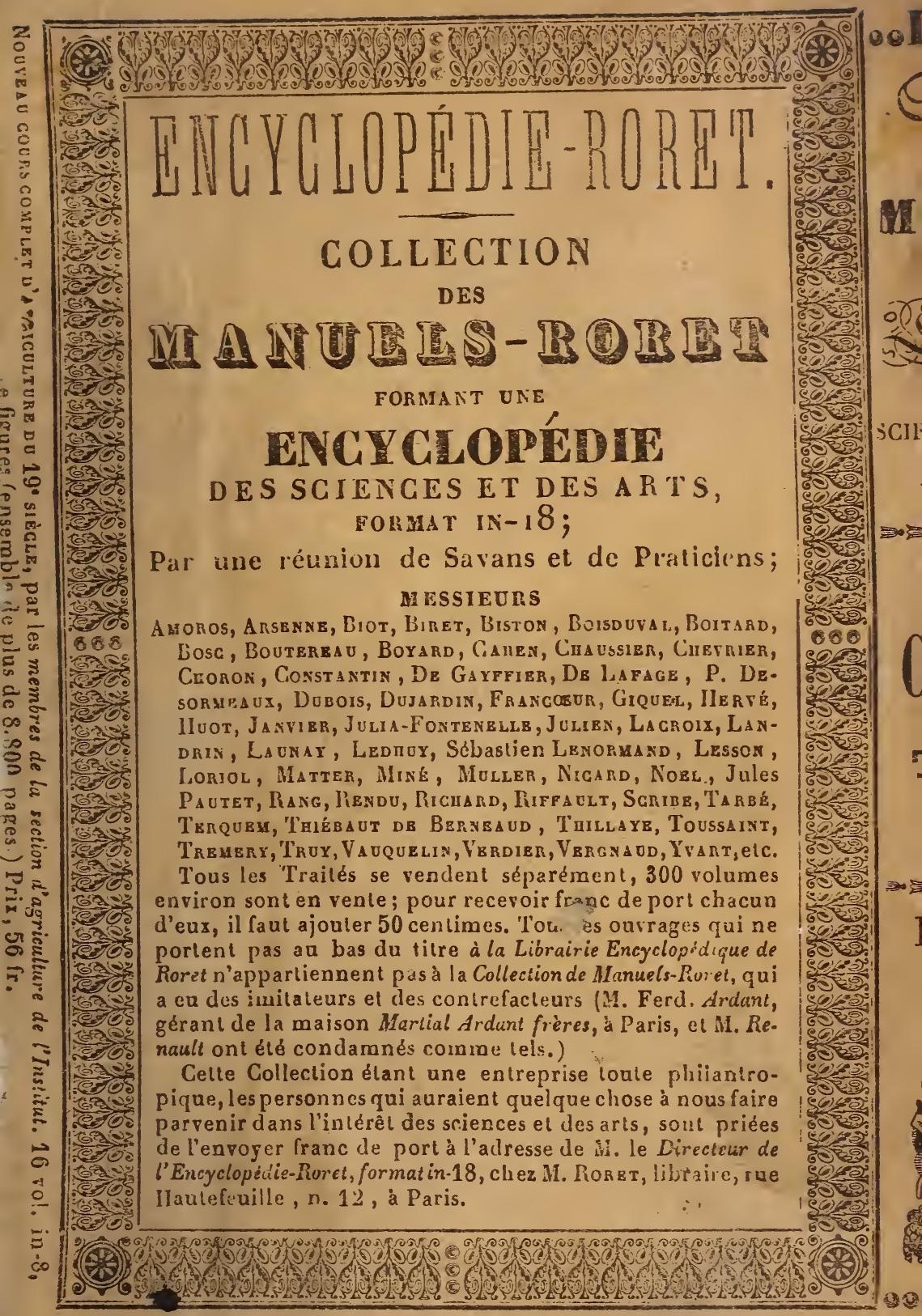

Traité des Arbras et Areostes, par Duhamel, Mirbel, Pairel. Ioiseleur-Deslenchamps.

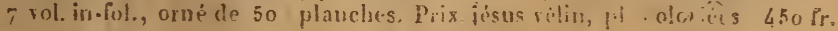



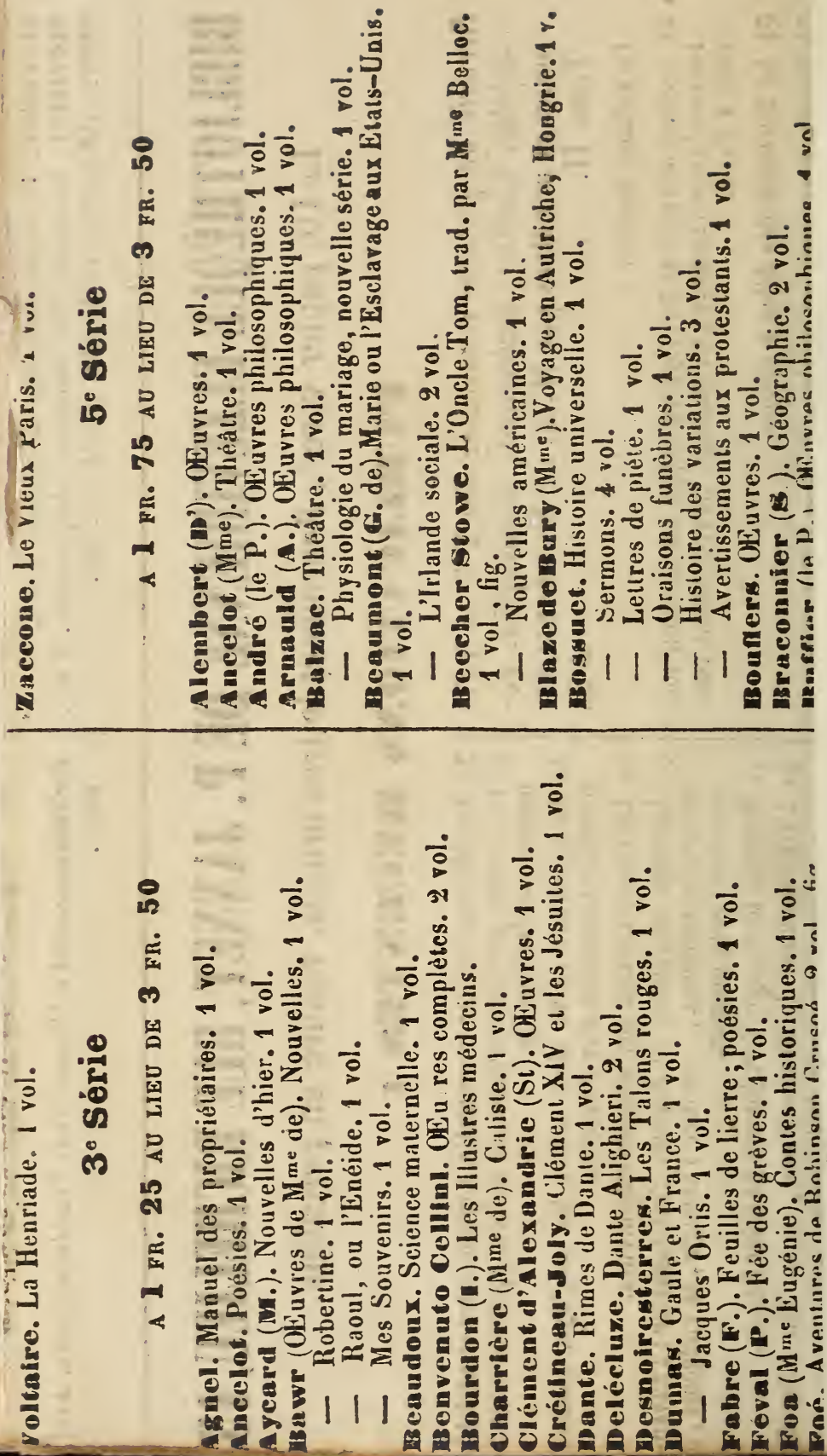





Deacidified using the Bookkeeper process. Neutralizing agent: Magnesium Oxide Treatment Date: Oct. 2012

\section{PreservationTechnologies} A WORLD LEADER IN COLLECTIONS PRESERVATION

111 Thomson Park Drive

Cramberty Township, PA 16066

(724) $779-2111$ 


\section{LIBRARY OF CONGRESS}

|||||||||| ||||||||||||||||||||||||||||||||||||||||||||||

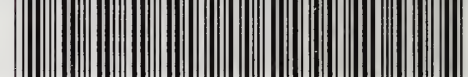

00008908138 